\title{
APERIODIC OSCILLATORY ASYMPTOTIC BEHAVIOR FOR SOME BIANCHI SPACETIMES
}

\author{
FRANÇOIS BÉGUIN
}

\begin{abstract}
We study the asymptotic behavior of vacuum Bianchi type A spacetimes close to their singularity. It has been conjectured that this behavior is driven by a certain circle map, called the Kasner map. As a step towards this conjecture, we prove that some orbits of the Kasner map do indeed attract some solutions of the system of ODEs which describes the behavior of vacuum Bianchi type A spacetimes. The orbits of the Kasner map for which we can prove such a result are those which are not periodic and do not accumulate on any periodic orbit. This shows the existence of Bianchi spacetimes with an aperiodic oscillatory asymptotic behavior.
\end{abstract}

\section{INTRODUCTION}

1.1. Bianchi spacetimes, the Wainwright-Hsu vector field and the Kasner map. A Bianchi spacetime is a cosmological spacetime which is spatially homogeneous. More precisely, it is a spacetime $(M, g)$ such that $M$ is diffeomorphic to the product $G \times I$ of a simply connected three-dimensional Lie group $G$ and an interval $I$ of the real line, and $g=h_{t}-d t^{2}$ where $h_{t}$ is a left-invariant riemannian metric on $G \times\{t\} \simeq G$ for every $t \in I$. A vacuum Bianchi spacetime is a Bianchi spacetime $(M, g)$ satisfying the vacuum Einstein equation $\operatorname{Ric}_{g}=0$. A type $A$ Bianchi spacetime is a Bianchi spacetime for which the corresponding three dimensional Lie group $G$ is unimodular. Note that the Lie group $G$ can be assumed to be simply connected without loss of generality.

A Bianchi spacetime can be described as a one-parameter family $\left(h_{t}\right)_{t \in I}$ of left-invariant riemannian metrics on a three-dimensional Lie group $G$. The space of left-invariant riemannian metrics on a given Lie group is finite-dimensional. Therefore, when restricted to the context of Bianchi spacetimes, the vacuum Einstein equation becomes an ODE on a finite dimensional phase space. A classical and convenient way to write this ODE is to use the so-called Wainwright-Hsu variables $\left(\Sigma_{1}, \Sigma_{2}, \Sigma_{3}, N_{1}, N_{2}, N_{3}\right)$. Very roughly, $N_{1}, N_{2}, N_{3}$ are the structure constants of the Lie algebra of the Lie group $G \times\{t\}$ in a certain basis, and $\Sigma_{1}, \Sigma_{2}, \Sigma_{3}$ are the components of the normalized traceless second fundamental form of $G \times\{t\}$ in the same basis. The corresponding phase space is the four-dimensional manifold

$$
\mathcal{B}:=\left\{\left(\Sigma_{1}, \Sigma_{2}, \Sigma_{3}, N_{1}, N_{2}, N_{3}\right) \in \mathbb{R}^{6} \mid \Sigma_{1}+\Sigma_{2}+\Sigma_{3}=0, \Omega=0\right\}
$$

where

$$
\Omega=6-\left(\Sigma_{1}^{2}+\Sigma_{2}^{2}+\Sigma_{3}^{2}\right)+\frac{1}{2}\left(N_{1}^{2}+N_{2}^{2}+N_{3}^{2}\right)-\left(N_{1} N_{2}+N_{1} N_{3}+N_{2} N_{3}\right) .
$$

Work supported by ANR project GEODYCOS. 
The vacuum Einstein equation is equivalent to the system of ODEs

$$
\left\{\begin{array}{l}
\Sigma_{1}^{\prime}=(2-q) \Sigma_{1}-R_{1} \\
\Sigma_{2}^{\prime}=(2-q) \Sigma_{2}-R_{2} \\
\Sigma_{3}^{\prime}=(2-q) \Sigma_{3}-R_{3} \\
N_{1}^{\prime}=-\left(q+2 \Sigma_{1}\right) N_{1} \\
N_{2}^{\prime}=-\left(q+2 \Sigma_{2}\right) N_{2} \\
N_{3}^{\prime}=-\left(q+2 \Sigma_{3}\right) N_{3}
\end{array}\right.
$$

where

$$
q=\frac{1}{3}\left(\Sigma_{1}^{2}+\Sigma_{2}^{2}+\Sigma_{3}^{2}\right)
$$

and

$$
\left\{\begin{array}{l}
R_{1}=\frac{1}{3}\left(2 N_{1}^{2}-N_{2}^{2}-N_{3}^{3}+2 N_{2} N_{3}-N_{1} N_{3}-N_{1} N_{2}\right) \\
R_{2}=\frac{1}{3}\left(2 N_{2}^{2}-N_{3}^{2}-N_{1}^{3}+2 N_{3} N_{1}-N_{2} N_{1}-N_{2} N_{3}\right) \\
R_{3}=\frac{1}{3}\left(2 N_{3}^{2}-N_{1}^{2}-N_{2}^{3}+2 N_{1} N_{2}-N_{3} N_{2}-N_{3} N_{1}\right)
\end{array}\right.
$$

(see e.g. [3] for the construction of the Wainwright-Hsu variables and the expression of the Einstein equation in these variables). In other words, vacuum Bianchi type A spacetimes can be seen as the solutions of the system of ODEs (1) on the four-dimensional manifold $\mathcal{B}$. We will call Wainwright-Hsu vector field, and denote by $X_{\mathcal{B}}$, the vector field on $\mathcal{B}$ associated to the system of ODEs (1). We will denote by $X_{\mathcal{B}}^{t}$ the time $t$ map of the flow of $X_{\mathcal{B}}$. To measure the distances on the phase space $\mathcal{B}$, we will use the riemannian metric $h=\left(d \Sigma_{1}\right)^{2}+\left(d \Sigma_{2}\right)^{2}+\left(d \Sigma_{3}\right)^{2}+\left(d N_{1}\right)^{2}+\left(d N_{2}\right)^{2}+\left(d N_{3}\right)^{2}$.

Remark 1.1. We have chosen the "anti-physical" time orientation. With this convention, Bianchi spacetimes are future incomplete, but not necessarily past incomplete. The main reason for this choice is that we want the so-called mixmaster attractor (see below) to be an attractor, rather than a repellor.

The phase space $\mathcal{B}$ admits a natural stratification, which is invariant under the flow of the WainwrightHsu vector field $X_{\mathcal{B}}$. There are six strata denoted by $\mathcal{B}_{\mathrm{I}}, \mathcal{B}_{\mathrm{II}}, \mathcal{B}_{\mathrm{VI}_{0}}, \mathcal{B}_{\mathrm{VII}_{0}}, \mathcal{B}_{\mathrm{VIII}}, \mathcal{B}_{\mathrm{IX}}$. These strata correspond to the different possible signs for the variables $N_{1}, N_{2}, N_{3}$. They also correspond to the different (isomorphism class of) simply connected unimodular three-dimensional Lie groups. The orbits of $X_{\mathcal{B}}$ contained in $\mathcal{B}_{\mathrm{I}}$ are called type I orbits, the orbits contained in $\mathcal{B}_{\text {II }}$ are called type II orbits, etc. The behavior of the type $\mathrm{I}, \mathrm{II}, \mathrm{VI}_{0}$ and $\mathrm{VII}_{0}$ orbits of $X_{\mathcal{B}}$ is very well understood. On the contrary, the behavior of the type VIII and IX orbits (which are the generic orbits in $\mathcal{B}$ ) is, at best, conjectural. In order to simplify the discussion we will focus our attention on the subset $\mathcal{B}^{+}$of $\mathcal{B}$ where the coordinates $N_{1}, N_{2}, N_{3}$ are non-negative:

$$
\mathcal{B}^{+}:=\left\{\left(\Sigma_{1}, \Sigma_{2}, \Sigma_{3}, N_{1}, N_{2}, N_{3}\right) \in \mathcal{B} \mid N_{1} \geq 0, N_{2} \geq 0, N_{3} \geq 0\right\} .
$$

Observe that this subset is invariant under the flow of $X_{\mathcal{B}}$.

The stratum $\mathcal{B}_{\mathrm{I}}$ is more frequently denoted by $\mathcal{K}$. This is a euclidean circle in $\mathcal{B} \subset \mathbb{R}^{6}$, called the Kasner circle. It is made of the points of $\mathcal{B}$ where $N_{1}=N_{2}=N_{3}=0$. This corresponds to the case 
where the Lie group $G$ is abelian (i.e. $G=\mathbb{R}^{3}$ ).

$$
\begin{aligned}
\mathcal{K}= & \left\{\left(\Sigma_{1}, \Sigma_{2}, \Sigma_{3}, N_{1}, N_{2}, N_{3}\right) \in \mathcal{B} \mid\right. \\
=\left\{\left(\Sigma_{1}, \Sigma_{2}, \Sigma_{3}, N_{1}, N_{2}, N_{3}\right) \in \mathbb{R}^{6} \mid\right. & \left.N_{1}=N_{2}=N_{3}=0\right\} \\
& \left.\Sigma_{1}+\Sigma_{2}+\Sigma_{3}=0, \Sigma_{1}^{2}+\Sigma_{2}^{2}+\Sigma_{3}^{2}=6\right\}
\end{aligned}
$$

The vector field $X_{\mathcal{B}}$ vanishes on $\mathcal{K}$. Therefore every type I orbit is a fixed point. There are three points on $\mathcal{K}$, called the special points or the Taub points, which play a very important role in every attempt to understand the behavior of the solution of the Wainwright-Hsu equations; these are the points for which $\left(\Sigma_{1}, \Sigma_{2}, \Sigma_{3}\right)$ is equal respectively to $(2,-1,-1),(-1,2,-1)$ and $(-1,-1,2)$; these points are usually denoted by $T_{1}, T_{2}, T_{3}$. For every point $p \in \mathcal{K}$, the derivative $D X_{\mathcal{B}}(p)$ has four distinct eigendirections: the direction $T_{p} \mathcal{K}$ of the Kanser circle corresponds to a zero eigenvalue (since $X_{\mathcal{B}}$ vanishes on $\mathcal{K}$ ), and the directions $\mathbb{R} \cdot \frac{\partial}{\partial N_{1}}(p), \mathbb{R} \cdot \frac{\partial}{\partial N_{2}}(p), \mathbb{R} \cdot \frac{\partial}{\partial N_{3}}(p)$ are respectively associated to the eigenvalues $-\left(2+2 \Sigma_{1}\right),-\left(2+2 \Sigma_{2}\right),-\left(2+2 \Sigma_{3}\right)$. When $p$ is one of the three special points $T_{1}, T_{2}, T_{3}$, two of the three eigenvalues $-\left(2+2 \Sigma_{1}\right),-\left(2+2 \Sigma_{2}\right),-\left(2+2 \Sigma_{3}\right)$ vanish; hence, the derivative of $D X_{\mathcal{B}}(p)$ has a triple zero eigenvalue. If $p$ is not one of the three special points $T_{1}, T_{2}, T_{3}$, then the eigenvalues $-\left(2+2 \Sigma_{1}\right),-\left(2+2 \Sigma_{2}\right),-\left(2+2 \Sigma_{3}\right)$ are pairwise distinct, two of them are negative, and the other one is positive; in other words, the derivative $D X_{\mathcal{B}}(p)$ has two distinct negative eigenvalue, a multiplicity one eigenvalue, and a positive eigenvalue.

The stratum $\mathcal{B}_{\text {II }}$ is two-dimensional. It is made of the points of $\mathcal{B}$ for which exactly two of the $N_{i}$ 's vanish. This corresponds to the case where the Lie group $G$ is isomorphic to the Heisenberg group.

$$
\mathcal{B}_{1} \cup \mathcal{B}_{\text {II }}=\left\{\left(\Sigma_{1}, \Sigma_{2}, \Sigma_{3}, N_{1}, N_{2}, N_{3}\right) \in \mathcal{B} \mid N_{1}=N_{2}=0 \text { or } N_{1}=N_{3}=0 \text { or } N_{2}=N_{3}=0\right\}
$$

One can easily check that $\mathcal{B}_{\mathrm{I}} \cup \mathcal{B}_{\mathrm{II}}$ is the union of three ellipsoids which intersect along the Kasner circle. The vector field $X_{\mathcal{B}}$ commutes with the transformation $\left(N_{1}, N_{2}, N_{3}\right) \mapsto\left(-N_{1},-N_{2},-N_{3}\right)$, so we can work in $\mathcal{B}_{\mathrm{II}} \cap \mathcal{B}^{+}$to simplify the discussion. The intersection $\mathcal{B}_{\mathrm{II}} \cap \mathcal{B}^{+}$is the union of three disjoint hemi-ellipsoids bounded by the Kasner circle. Type II orbits can be calculated explicitly (see for example [3]). It appears that every type II is a heteroclinic orbit connecting a point of $\mathcal{K} \backslash\left\{T_{1}, T_{2}, T_{3}\right\}$ to another point of $\mathcal{K} \backslash\left\{T_{1}, T_{2}, T_{3}\right\}$. Moreover, for every point $p \in \mathcal{K} \backslash\left\{T_{1}, T_{2}, T_{3}\right\}$, there is exactly one type II orbit in $\mathcal{B}^{+}$which "takes off" at $p$ (this orbit is tangent to the eigendirection associated to the positive eigenvalue of $D X_{\mathcal{B}}(p)$ ) and two type II orbits in $\mathcal{B}^{+}$which "land" at $p$ (these orbits are tangent to the eigendirections associated to the negative eigenvalues of $D X_{\mathcal{B}}(p)$ ).

The Kasner map $f: \mathcal{K} \rightarrow \mathcal{K}$ is defined as follows. Consider a point $p \in \mathcal{K}$. If $p$ is one of the three special points $T_{1}, T_{2}, T_{3}$, then $f(p)=p$. Otherwise, one considers the unique type II orbit which "springs up" at $p$; this orbit connects $p$ to another point $p^{\prime} \in \mathcal{K}$; this point $p^{\prime}$ is by definition the image of $p$ under $f$. For every $p \in \mathcal{K} \backslash\left\{T_{1}, T_{2}, T_{3}\right\}$, we will denote by $\mathcal{O}_{p, f(p)}$ the unique type II orbit connecting the point $p$ to the point $f(p)$ in $\mathcal{B}^{+}$. Since there are exactly two type II orbits in $\mathcal{B}^{+}$which land at a given point $p \in \mathcal{K}$, the Kasner map is two-to-one. The exact computation of the type II orbits of $X_{\mathcal{B}}$ yields a nice geometric description of the Kasner map $f$. Consider the equilateral triangle which is tangent to $\mathcal{K}$ at the three special points $T_{1}, T_{2}, T_{3}$. Denote by $M_{1}, M_{2}, M_{3}$ the vertices of this triangle. For $p \in \mathcal{K} \backslash\left\{T_{1}, T_{2}, T_{3}\right\}$, let $M_{i}$ be the vertex of $\mathcal{K}$ which is the closest to $p$. The line $\left(p M_{i}\right)$ and intersects the circle $\mathcal{K}$ at two points: the point $p$ and the point $f(p)$. See figure 1 . Using this geometric description, it is easy to see $f$ is $C^{1}$. Moreover, the Kasner map $f$ is non-uniformly expanding. Indeed, the norm of the derivative of $f$ (calculated with respect to the metric on $\mathcal{K}$ induced by the riemannian metric $h$ ) at a point $p \in \mathcal{K}$ is equal to 1 if $p$ is one of the three special points $T_{1}, T_{2}, T_{3}$, and is strictly bigger than 1 if $p$ 


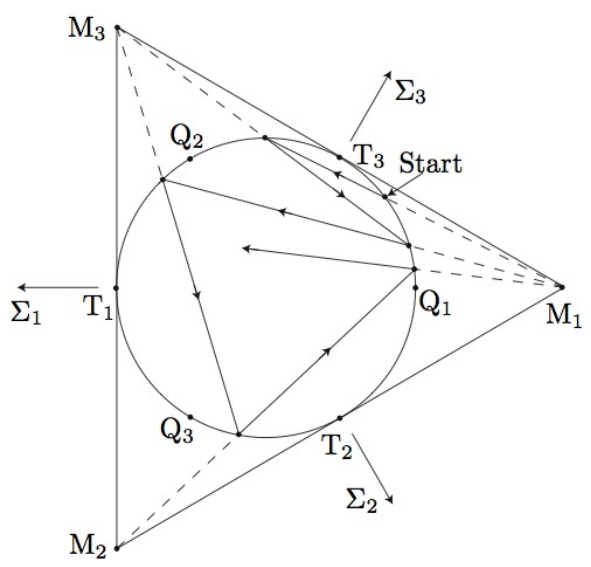

FIGURE 1. Construction of an orbit of the Kasner map

is not one of these three special points. It follows that, for every compact set $C$ in $\mathcal{K} \backslash\left\{T_{1}, T_{2}, T_{3}\right\}$ there exists a constant $\nu_{C}>1$ such that $\|\mid D f(p)\| \|_{h} \geq \nu_{C}$ for every $p$ in $C$.

The strata $\mathcal{B}_{\mathrm{VI}_{0}}$ and $\mathcal{B}_{\mathrm{VII}_{0}}$ are three-dimensional. The stratum $\mathcal{B}_{\mathrm{VI}_{0}}$ is made of the points of $\mathcal{B}$ for which exactly one of the $N_{i}$ 's is equal to zero, the two others being of different signs. This corresponds to the case where the Lie group $G$ is isomorphic to $\operatorname{Isom}\left(\mathbb{R}^{1,1}\right)=\mathrm{O}(1,1) \ltimes \mathbb{R}^{2}$. This stratum is disjoint from $\mathcal{B}^{+}$. The stratum $\mathcal{B}_{\mathrm{VII}_{0}}$ is made of the points of $\mathcal{B}$ for which exactly one of the $N_{i}$ 's is equal to zero, the two others being of the same sign. This corresponds to the case where the Lie group $G$ is isomorphic to $\operatorname{Isom}\left(\mathbb{R}^{2}\right)=O(2) \ltimes \mathbb{R}^{2}$.

Finally the strata $\mathcal{B}_{\text {VIII }}$ and $\mathcal{B}_{\text {IX }}$ are four-dimensional (these are open subsets of $\mathcal{B}$ ). The stratum $\mathcal{B}_{\text {VIII }}$ is made of the points of $\mathcal{B}$ for which the $N_{i}$ 's are non-zero and do not have the same sign. It corresponds to the case where the Lie group $G$ is isomorphic to the universal cover of $\operatorname{SL}(2, \mathbb{R})$. It is disjoint from $\mathcal{B}^{+}$. The stratum $\mathcal{B}_{\text {IX }}$ is made of the points of $\mathcal{B}$ for which the $N_{i}$ 's are either all positive, or all negative. This corresponds to the case where the Lie group $G$ is isomorphic to $\mathrm{SO}(3, \mathbb{R})$.

1.2. Statement of the main results of the paper. Misner has conjectured that the dynamics of type IX orbits of the Wainwright-Hsu vector field $X_{\mathcal{B}}$ "is driven" by the Kasner map ([5]). The idea is that every type IX orbit should eventually approach the so-called mixmaster attractor $\mathcal{A}:=\mathcal{K} \cup \mathcal{B}_{\mathrm{II}}$, and then "follow" a heteroclinic chain $q \rightarrow \mathcal{O}_{q, f(q)} \rightarrow f(q) \rightarrow \mathcal{O}_{f(q), f^{2}(q)} \rightarrow$. . Conversely, every heteroclinic chain as above should attract some type IX orbits of $X_{\mathcal{B}}$. Moreover, "generic" type IX orbits should be attracted by "generic" heteroclinic chains. This would imply that the behavior of generic type IX orbits of $X_{\mathcal{B}}$ is determined by the behavior of generic orbits of the Kasner map. See [3] for a detailed discussion of various possible precise statements for this conjecture.

In 2001, Ringström proved that $\mathcal{A}:=\mathcal{K} \cup \mathcal{B}_{\text {II }}$ is indeed an global attractor: the distance from almost every type IX orbit of the Wainwright-Hsu vector field $X_{\mathcal{B}}$ to $\mathcal{A}$ tends to 0 as the time goes to $\infty$ ([9], see also [4]). Ringström's theorem has important consequences, such as the divergence of the curvature in Bianchi spacetimes as one approaches their past (or future) singularity. Nevertheless, this theorem does not tell anything on the relation between the dynamics of Bianchi orbits and the dynamics the Kasner 
map. The purpose of the present paper is to prove that "there are many points $q \in \mathcal{K}$ such that the heteroclinic chain $q \rightarrow \mathcal{O}_{q, f(q)} \rightarrow f(q) \rightarrow \mathcal{O}_{f(q), f^{2}(q)} \rightarrow \ldots$ does attract some type IX orbits of $X_{\mathcal{B}}$ ".

Definition 1.2. For $q \in \mathcal{K}$, we call stable manifold of $q$, and we denote by $W^{s}(q)$, the set of all points $r \in \mathcal{B}$ for which there exists an increasing sequence of real numbers $\left(t_{n}\right)_{n \geq 0}$ such that

$$
\operatorname{dist}\left(X_{\mathcal{B}}^{t_{n}}(r), f^{n}(q)\right) \underset{n \rightarrow \infty}{\longrightarrow} 0
$$

and such that the Hausdorff distance between the piece of orbit $\left\{X_{\mathcal{B}}^{t}(r) ; t_{n} \leq t \leq t_{n+1}\right\}$ and the typeII heteroclinic orbit $\mathcal{O}_{f^{n}(q), f^{n+1}(q)}$ tends to 0 as $n$ goes to $+\infty$.

A subset $C$ of $\mathcal{K}$ is said to be forward-invariant if $f(C) \subset C$. It is said to be aperiodic if it does not contain any periodic orbit for $f$. Our main result can be stated as follows:

Theorem 1.3. If $q \in \mathcal{K}$ is contained in a closed forward-invariant aperiodic subset of $\mathcal{K}$, then the intersection of the stable manifold $W^{s}(q)$ with $\mathcal{B}_{I X}$ is non-empty. More precisely, $W^{s}(q) \cap \mathcal{B}_{I X}$ contains a $C^{1}$ embedded three-dimensional closed disc. This disc can be chosen to depend continuously on $q$ (for the $C^{1}$ topology on the space of $C^{1}$ embeddings of the closed unit disc of $\mathbb{R}^{3}$ in $\mathcal{B}$ ) when $q$ ranges in a closed forward-invariant aperiodic subset of $\mathcal{K}$.

Remark 1.4. Actually, one can prove that $W^{s}(q) \cap \mathcal{B}_{\text {IX }}$ is an injectively immersed open disc, and that this depends continuously on $q$ (for the compact-open $C^{1}$ topology on the space of $C^{1}$ immersions of the open unit disc of $\mathbb{R}^{3}$ in $\mathcal{B}_{\mathrm{IX}}$ ) when $q$ ranges in a closed forward-invariant aperiodic subset of $\mathcal{K}$. Note that $W^{s}(q) \cap \mathcal{B}_{\mathrm{IX}}$ is never a properly embedded open disc.

Remark 1.5. We decided to focus on type IX orbits for sake of simplicity. Nevertheless, the analog of theorem 1.3 for $\mathcal{B}_{\text {VIII }}$ instead of $\mathcal{B}_{\text {IX }}$ is also true. The proof is exactly the same: one just needs to replace the set $\mathcal{B}^{+}$by the set $\mathcal{B}^{(-,+,+)}:=\mathcal{B} \cap\left\{N_{1} \leq 0\right\} \cap\left\{N_{2} \geq 0\right\} \cap\left\{N_{3} \geq 0\right\}$.

Observe that the hypothesis of theorem 1.3 is satisfied by a dense set of points in $\mathcal{K}$ :

Proposition 1.6. The union of all the closed forward-invariant aperiodic subsets of $\mathcal{K}$ is in dense in $\mathcal{K}$.

Remark 1.7. Let $E$ be the union of all the closed forward-invariant aperiodic subsets of $\mathcal{K}$. According to the above proposition, $E$ is dense in $\mathcal{K}$. Observe nevertheless that $E$ is a "small" subset of $\mathcal{K}$ both from the topological viewpoint (it is a meager set) and from the measurable viewpoint (it has zero Lebesgue measure). Also observe that theorem 1.3 does not tell that $W^{s}(q)$ depends continuously on $q$ when $q$ ranges in $E$. So, we do not know whether the union of all the stable manifolds $W^{s}(q)$, where $q$ ranges $E$, is dense in $\mathcal{B}$ (or in an open subset of $\mathcal{B}$ ) or not.

The origin of the hypothesis of theorem 1.3 is purely technical: if $p$ belongs to a closed forwardinvariant aperiodic subset of $\mathcal{K}$, we can find a coordinate system in which the vector field $X_{\mathcal{B}}$ depends linearly of all the coordinates but one; this makes the estimates on the flow of $X_{\mathcal{B}}$ near such a point much easier. We do not know if such linearizing coordinate systems exists near a point of $\mathcal{K}$ which is periodic or preperiodic under the Kasner map. Nevertheless, it should be noticed that the estimates on the flow of $X_{\mathcal{B}}$ that we need to construct stable manifolds are much weaker than the existence of a linearizing coordinate system. So, we do think that theorem 1.3 can be extended to any point $q \in \mathcal{K}$ such that the orbit of $q$ under the Kasner map does not accumulate one any of the three special points $T_{1}, T_{2}, T_{3}$. Understanding the behavior of the orbits which pass arbitrary close to the three special points seems to be a much harder problem. 
Remark 1.8. A consequence of theorem 1.3 (and proposition 1.6) is that the Wainwright-Hsu vector field is, at least partially, sensitive to initial conditions: there is a dense subset $E$ of the Kasner circle $\mathcal{K}$ such that, for every point $q \in E$, arbitrarily closed to $q$, one can find two points $r_{1}, r_{2} \in \mathcal{B}_{\text {IX }}$ such that the orbits of $r_{1}$ and $r_{2}$ will not have the same "asymptotic behavior" (for example, $\limsup _{t \rightarrow+\infty} \operatorname{dist}_{h}\left(X_{\mathcal{B}}^{t}\left(r_{1}\right), X_{\mathcal{B}}^{t}\left(r_{2}\right)\right)>1 / 10$.

As we were finishing to write the present paper, M. Georgi, J. Härterich, S. Liebscher and K. Webster put on arXiv a preprint in which they prove that the period three orbits of $f$ admit non-trivial stable manifolds ([2]). At the end of this preprint, they claim that their techniques can be used to extend their result to any periodic orbit of $f$, and even to any orbit of $f$ which does not accumulate one any of the three special points $T_{1}, T_{2}, T_{3}$. Such a extension would imply our result.

1.3. Idea of the proof and organization of the paper. Let us try to sketch the key idea of the proof of our main theorem 1.3. Let $C$ be a closed forward-invariant aperiodic subset of the Kasner circle, and denote by $\widehat{C}$ the union of $C$ and all the type II orbits connecting two points of $C$ in $\mathcal{B}^{+}$. We will construct a kind of Poincaré section adapted to $\widehat{C}$ : a hypersurface with boundary $M_{C}$ which intersects transversally every type II orbit connecting two points of $C$ in $\mathcal{B}^{+}$. Theorem 1.3 will follow from the existence of non-trivial local stable manifolds for the Poincaré map $\Phi_{C}: M_{C} \rightarrow M_{C}$. We will use a classical stable manifold theorem for hyperbolic compact set; so, we will be left to prove that the compact set $\widehat{C} \cap M_{C}$ is a hyperbolic set for the Poincaré map $\Phi_{C}$. The key point is to understand the behavior of the orbits of the Wainwright-Hsu vector field $X_{\mathcal{B}}$ close to a point $p \in C$. Roughly speaking, we will prove the following: when an orbit of $X_{\mathcal{B}}$ passes close to a point $p \in C$, the distance of this orbit to the mixmaster attractor $\mathcal{A}=\mathcal{K} \cup \mathcal{B}_{\text {II }}$ decreases super-linearly, while the drift of this orbit tangentially to $\mathcal{A}$ is as small as wanted. We do not have any precise control of what happens to the orbits of $X_{\mathcal{B}}$ far from the Kasner circle, but we do not need to. Indeed, the duration of the excursion of the orbits of $X_{\mathcal{B}}$ outside any given neighborhood of $\mathcal{K}$ is universally bounded. A consequence is that everything that happens far from $\mathcal{K}$ is dominated by the super-linear contraction of the distance to $\mathcal{A}$ that occurs when an orbit passes close to $C$. This will be enough to obtain the desired hyperbolicity result. As already explained, the reason why we need to consider an aperiodic subset of $\mathcal{K}$ is purely technical: close to a point of $\mathcal{K}$ which is not preperiodic under the Kasner map, we have a "nice" coordinate system which is convenient to study precisely the behavior of the orbits of $X_{\mathcal{B}}$.

Let us now explain the organization of the paper. Consider a vector field $X$ on some manifold and a point $p$ such that $X(p)=0$. We say that $X$ satisfies Sternberg's condition at $p$ if the non purely imaginary eigenvalues of $D X(p)$, counted with multiplicities, are linearly independent over $\mathbb{Q}$. Takens has proved a generalization of Sternberg's theorem which states that, if $X$ satisfies Sternberg's condition at $p$, there exists a $C^{1}$ local "linearizing" coordinate system for $X$ on a neighborhood of $p$. A precise statement of this theorem will be given in section 2 . The purpose of section 3 is to apply Takens' theorem to the Wainwright-Hsu vector field. It will be proved that the Wainwright-Hsu vector field $X_{\mathcal{B}}$ satisfies Sternberg's condition at some point $p$ of the Kasner circle if and only if $p$ is not preperiodic under the Kasner map. In section 4, the "linearizing" coordinate system provided by Takens' theorem will be used to study the behavior of the orbits of the Wainwright-Hsu vector field close to a point of the Kasner circle which is not periodic under the Kasner map. Now consider a closed forward-invariant aperiodic subset $C$ of the Kasner circle $\mathcal{K}$ is considered, and denote by $\widehat{C}$ the union of $C$ and all the type II orbits connecting two points of $C$ in $\mathcal{B}^{+}$. A Poincaré section $M_{C}$ adapted to $\widehat{C}$ will be constructed in section 5; we will 
denote by $\Phi_{C}$ the corresponding Poincaré map. The existence of non-trivial stable manifolds for the corresponding Poincaré map $\Phi_{C}: M_{C} \rightarrow M_{C}$ will be proved in section 6, using the results of section 4 . The proof of theorem 1.3 will be completed in section 7 . Finally, the last section of the paper is devoted to the proof of proposition 1.6.

Acknowledgements. I would like to thank Lars Andersson for some stimulating discussion, and for his encouragements to write the present paper.

\section{TAKENS' LINEARIZATION THEOREM}

Let $X$ be a $C^{\infty}$ vector field on some manifold $\mathcal{M}$, and $p$ be a point in $\mathcal{M}$ such that $X(p)=0$. The linear space $T_{p} \mathcal{M}$ admits a unique decomposition

$$
T_{p} \mathcal{M}=E^{s} \oplus E^{c} \oplus E^{u}
$$

where $E^{s}, E^{c}, E^{u}$ are $D X(p)$-invariant linear subspaces, the eigenvalues of $D X(p)_{\mid E^{s}}$ have negative real parts, the eigenvalues of $D X(p)_{\mid E^{c}}$ are purely imaginary, and the eigenvalues of $D X(p)_{\mid E^{u}}$ have positive positive. We denote by $s, c$ and $u$ the dimensions of the linear subspaces $E^{s}, E^{c}$ and $E^{u}$.

Definition 2.1. The vector field $X$ satisfies Sternberg's condition at $p$ if the eigenvalues of $D X(p)_{\mid E^{s} \oplus E^{u}}$ (counted with multiplicities) are linearly independent over $\mathbb{Q}$.

F. Takens as proved the following generalization of the classical Sternberg linearization theorem:

Theorem 2.2 (see [10], page 144). Assume that $X$ satisfies Sternberg's condition at $p$. Then, for every $r \geq 0$, one can find a neighborhood $U$ and $a C^{r}$ coordinate system $\left(x_{1}, \ldots, x_{s}, y_{1}, \ldots, y_{c}, z_{1}, \ldots, z_{u}\right)$ on $U$ centered at $p$, such that, in this coordinate system, $X$ reads:

$$
X=\sum_{i, j=1}^{s} a_{i, j}\left(y_{1}, \ldots, y_{c}\right) x_{j} \frac{\partial}{\partial x_{i}}+\sum_{i=1}^{c} \phi_{i}\left(y_{1}, \ldots, y_{c}\right) \frac{\partial}{\partial y_{i}}+\sum_{i, j=1}^{u} b_{i, j}\left(y_{1}, \ldots, y_{c}\right) z_{j} \frac{\partial}{\partial z_{i}}
$$

where the eigenvalues of the matrix $\left(a_{i, j}(0, \ldots, 0)\right)_{1 \leq i, j \leq s}$ have negative real parts, the eigenvalues of the matrix $\left(\frac{\partial \phi_{i}}{\partial y_{j}}(0, \ldots, 0)\right)_{1 \leq i, j \leq c}$ are purely imaginary, and the eigenvalues of the matrix $\left(b_{i, j}(0, \ldots, 0)\right)_{1 \leq i, j \leq u}$ have positive real parts.

Note that, in general, the size of the neighborhood $U$ does depend on the integer $r$, and it is not possible to find any $C^{\infty}$ local coordinate system centered at $p$ such that (3) holds. In this sense, Takens' theorem is not a true generalization of Sternberg's theorem.

Also observe that the name "Takens' linearization theorem" is slightly incorrect: indeed, the vector field $X$ is not linear in the $\left(x_{1}, \ldots, x_{s}, y_{1}, \ldots, y_{c}, z_{1}, \ldots, z_{u}\right)$ coordinate system. Nevertheless, $X$ depends linearly on the coordinates $x_{1}, \ldots, x_{s}$ and $z_{1}, \ldots, z_{u}$. Also note that the submanifold defined by the equation $\left(y_{1}, \ldots, y_{c}\right)=(0, \ldots, 0)$ is invariant under the flow of $X$, and that the restriction of $X$ to this submanifold is linear. For $\left(\zeta_{1}, \ldots, \zeta_{c}\right) \neq(0, \ldots, 0)$, the submanifold defined by the equation $\left(y_{1}, \ldots, y_{c}\right)=\left(\zeta_{1}, \ldots, \zeta_{c}\right)$ is not invariant under the flow of $X$, but the projection of $X$ on the tangent space of this submanifold is linear.

Of course, equality (3) together with the signs of the real parts of the eigenvalues of the matrices $\left(a_{i, j}(0, \ldots, 0)\right)_{1 \leq i, j \leq s},\left(\frac{\partial \phi_{i}}{\partial y_{j}}(0, \ldots, 0)\right)_{1 \leq i, j \leq c}$ and $\left(b_{i, j}(0, \ldots, 0)\right)_{1 \leq i, j \leq u}$ implies that: 
- the vectors $\frac{\partial}{\partial x_{1}}(p), \ldots, \frac{\partial}{\partial x_{s}}(p)$ span the linear subspace $E^{s}$,

- the vectors $\frac{\partial}{\partial y_{1}}(p), \ldots, \frac{\partial}{\partial y_{c}}(p)$ span the linear subspace $E^{c}$,

- the vectors $\frac{\partial}{\partial z_{1}}(p), \ldots, \frac{\partial}{\partial z_{u}}(p)$ span the linear subspace $E^{u}$.

\section{LineARIZATION OF WAINWRIGHT-HSU VECTOR FIELD NEAR A POINT OF THE KASNER CIRCLE WHICH IS NOT PREPERIODIC UNDER THE KASNER MAP}

The purpose of this section is to apply Takens' theorem to the Wainwright-Hsu vector field at a point of the Kasner circle. For this purpose, we will need to characterize the points $p$ on the Kasner circle such that the Wainwirght-Hsu vector field $X_{\mathcal{B}}$ satisfies Sternberg's condition at $p$. We will see that these are exactly the points $p \in \mathcal{K}$ which are not preperiodic under the Kasner map $f$. In order to relate the arithmetic properties of the eigenvalues of the derivative of $D X_{\mathcal{B}}(p)$ and the behavior of the orbit of $p$ under $f$, we will use the so-called Kasner parameter.

3.1. Kasner parameter. Let $q=\left(\Sigma_{1}, \Sigma_{2}, \Sigma_{3}, 0,0,0\right)$ be a point of the Kasner circle. The Kasner parameter of $q$ is the unique real number $u=u(q) \in[1, \infty]$ which satisfies the following equality:

$$
\left(2+\Sigma_{1}\right)\left(2+\Sigma_{2}\right)\left(2+\Sigma_{3}\right)=\frac{-216 u^{2}(1+u)^{2}}{\left(1+u+u^{2}\right)^{3}} .
$$

The map $q \mapsto u(q)$ is not one-to-one. Nevertheless, the point $q$ is characterized by its Kasner parameter up to permutations of the coordinates $\Sigma_{1}, \Sigma_{2}, \Sigma_{3}$. More precisely, equality (4) together with the equation of the Kasner circle (2) imply that:

$$
\left(2+\Sigma_{1}, 2+2 \Sigma_{2}, 2+2 \Sigma_{3}\right) \underset{\text { permutation }}{\stackrel{\text { up to }}{=}}\left(\frac{-6 u}{1+u+u^{2}}, \frac{6(1+u)}{1+u+u^{2}}, \frac{6 u(1+u)}{1+u+u^{2}}\right) .
$$

Note that $u=\infty$ if and only if $q$ is one of the three special points $T_{1}, T_{2}, T_{3}$. The main advantage of the Kasner parameter is the fact that the Kasner map $f$ admits a nice expression in terms of this parameter: for every $q \in \mathcal{K}$, one has $u(f(q))=\bar{f}(u(q))$ where $\bar{f}:[1,+\infty] \rightarrow[1, \infty]$ is defined by

$$
\bar{f}(u)= \begin{cases}\infty & \text { if } u=1 \text { or } \infty \\ u-1 & \text { if } u \geq 2 \\ \frac{1}{u-1} & \text { if } 1<u \leq 2\end{cases}
$$

(see, for example, [3]).

3.2. Characterization of the points of the Kasner circle where Sternberg's condition is satisfied. The proposition below gives a necessary and sufficient condition for the Wainwright-Hsu vector field $X_{\mathcal{B}}$ to satisfy Sternberg's condition at a point $p \in \mathcal{K}$, in terms of the behavior of the orbit of $p$ under the Kasner map. The hypothesis of our main theorem 1.3 comes directly from this condition.

Proposition 3.1. Let $p$ be a point of the Kasner circle $\mathcal{K}$ which is not one of the three special points $T_{1}, T_{2}, T_{3}$. The three following conditions are equivalent :

(1) the vector field $X_{\mathcal{B}}$ satisfies Sternberg's condition at $p$;

(2) the Kasner parameter $u(p)$ is neither a rational number, nor a quadratic irrational number ; 
(3) the orbit of $p$ under the Kasner map $f$ is not preperiodic.

Proof. Denote by $\left(\Sigma_{1}, \Sigma_{2}, \Sigma_{3}, 0,0,0\right)$ the coordinates of $p$. Since $p$ is not one of the three special points, the derivative $D X_{\mathcal{B}}(p)$ has two distinct negative eigenvalues, one zero eigenvalue, and one positive eigenvalue. The three non-zero eigenvalues of $D X_{\mathcal{B}}(p)$ are equal to $-\left(2+\Sigma_{1}\right),-\left(2+\Sigma_{2}\right)$ and $-\left(2+\Sigma_{3}\right)$.

Let us prove the equivalence between (1) and (2). The vector field $X_{\mathcal{B}}$ satisfies Sternberg's condition at $p$ if and only if the real numbers $-\left(2+\Sigma_{1}\right),-\left(2+\Sigma_{2}\right)$ and $-\left(2+\Sigma_{3}\right)$ are linearly independent over $\mathbb{Q}$. Using formula (5), one sees that this is equivalent to the fact that the real numbers $u(p), 1+u(p)$ and $u(p)(1+u(p))$ are independent over $\mathbb{Q}$. Clearly, this is equivalent to the fact the real number $u(p)$ is neither a rational number, nor a quadratic irrational number.

Now, let us prove the equivalence between (2) and (3). Recall that, for every $q$ on the Kasner circle, one has $u(f(q))=\bar{f}(u(q))$ where $\bar{f}:[1,+\infty] \rightarrow[1, \infty]$ is given by (6). Observe that both the set of rational numbers and the set of irrational numbers are invariant under $\bar{f}$. So we can treat the case where $u(p)$ is rational and the case where $u(p)$ is irrational separately.

First consider the case where $u(p)$ is rational. Then it is very easy to prove that the orbit of $u(p)$ under $\bar{f}$ "ends up" at $\infty$. Now recall that $u(q)=\infty$ if and only if $q$ is one of the three special points. Hence the orbit of $p$ under $f$ "ends up" at one of the three special points. In particular, the orbit of $p$ is preperiodic.

Now consider the case where $u(p)$ is irrational. Looking again at (6), one sees that the orbit of $u(p)$ under $\bar{f}$ returns an infinite number of times in the interval $(1,2]$. Let $k:(1, \infty) \backslash \mathbb{Q} \rightarrow \mathbb{N} \backslash\{0\}$ be the return time function of $\bar{f}$ in the interval $(1,2]$, and $\bar{F}:(1,2] \backslash \mathbb{Q} \rightarrow(1,2] \backslash \mathbb{Q}$ be the first return map of $\bar{f}$ in the interval $(1,2]$, that is

$$
k(u)=\inf \left\{n>0 \text { such that } \bar{f}^{n}(u) \in(1,2]\right\} \quad \text { and } \quad \bar{F}(u)=\bar{f}^{k(u)}(u) .
$$

Then,

$$
k(u)=\left\lfloor\frac{1}{u-1}\right\rfloor-1 \text { and } \bar{F}(u)=\left\{\frac{1}{u-1}\right\}+1,
$$

where $\lfloor x\rfloor$ is the integer part of $x$ and $\{x\}:=x-\lfloor x\rfloor$ is the fractional part of $x$. The point $p$ is preperiodic under the Kasner map $f$ if and only if either $u(p)$ is a preperiodic under $\bar{f}$, that is if and only if $\bar{f}^{k(u(p))}(u(p))$ is preperiodic under $\bar{F}$. Now observe that $\bar{F}$ is just the Gauss map $u \mapsto\left\{\frac{1}{u}\right\}$ conjugated by the translation $u \mapsto u+1$, and that $k(u-1)+1$ is the first term of the continuous development fraction of $u$. On the one hand, the preperiodic points of the Gauss map $u \mapsto\left\{\frac{1}{u}\right\}$ are exactly the real numbers $u \in(0,1]$ such that the sequence of integers which appear in the continuous fraction development of $u$ is preperiodic. On the other hand, it is well-known that the continuous fraction development of $u \in \mathbb{R}$ is preperiodic if and only if $u$ is a quadratic irrational number. This shows that the orbit of $p$ is a preperiodic under the Kasner map $f$ if and only if $u(p)$ is a quadratic irrational number.

3.3. Linearization of the Wainwright-Hsu vector field. According to proposition 3.1, if $p$ is not a preperiodic point for the Kasner map, the hypotheses of Takens linearization theorem 2.2 are satisfied by $X_{\mathcal{B}}$ at $p$. This theorem provides us with a local coordinate system on a neighborhood of $p$ in which $X_{\mathcal{B}}$ is "almost linear":

Proposition 3.2. Let $p$ be a point of the Kasner circle $\mathcal{K}$ which is not preperiodic under the Kasner map (in particular, $p$ is not a special point). Then there exists a neighborhood $U$ of $p$ in $\mathcal{B}$ and a $C^{1}$ coordinate 
system $\left(x_{1}, x_{2}, y, z\right)$ on $U$, centered at $p$, and such that, in this coordinate system, $X_{\mathcal{B}}$ reads:

$$
X_{\mathcal{B}}\left(x_{1}, x_{2}, y, z\right)=\lambda_{1}^{s}(y) x_{1} \frac{\partial}{\partial x_{1}}+\lambda_{2}^{s}(y) x_{2} \frac{\partial}{\partial x_{2}}+\lambda^{u}(y) z \frac{\partial}{\partial z}
$$

where $\lambda_{1}^{s}(y)<\lambda_{2}^{s}(y)<0$ and $\lambda^{u}(y)>0$ for every $y$.

Remarks 3.3. Let $U$ be a neighborhood of the point $p$ and $\left(x_{1}, x_{2}, y, z\right)$ be a $C^{1}$ coordinate system on $U$ centered at $p$, such that $X_{\mathcal{B}}$ satisfies (7) with $\lambda_{1}^{s}(y)<\lambda_{2}^{s}(y)<0$ and $\lambda^{u}(y)>0$. Then:

(i) The vector field $X_{\mathcal{B}}$ vanishes on the one-dimensional submanifold $\left\{x_{1}=x_{2}=z=0\right\}$ and nowhere else. It follows that this submanifold is the intersection of the Kasner circle with $U$ :

$$
\mathcal{K} \cap U=\left\{x_{1}=x_{2}=z=0\right\} .
$$

(ii) For every $y$, the real numbers $\lambda_{1}^{s}(y), \lambda_{2}^{s}(y), \lambda^{u}(y)$ are the three non-zero eigenvalues of the derivative of $X_{\mathcal{B}}$ at the point of coordinates $(0,0, y, 0) \in \mathcal{K}$. Recall that this derivative also has one zero eigenvalue (corresponding to the direction of the Kasner circle).

(iii) For every $\zeta$, the three-dimensional sub-manifold $\{y=\zeta\}$ is invariant under the flow of $X_{\mathcal{B}}$ and the restriction of $X_{\mathcal{B}}$ to this submanifold is linear.

(iv) The three-dimensional submanifolds $\left\{x_{1}=0\right\},\left\{x_{2}=0\right\}$ and $\{z=0\}$ are invariant under the flow of $X_{\mathcal{B}}$, and contain $\mathcal{K} \cap U$. It follows that these submanifolds coincide up to permutation with the submanifolds $\left\{N_{1}=0\right\},\left\{N_{2}=0\right\}$ and $\left\{N_{3}=0\right\}$. As a consequence, the twodimensional submanifolds $\left\{x_{1}=x_{2}=0\right\},\left\{x_{1}=z=0\right\}$ and $\left\{x_{2}=z=0\right\}$ coincide up to permutation with the submanifolds $\left\{N_{1}=N_{2}=0\right\},\left\{N_{1}=N_{3}=0\right\}$ and $\left\{N_{2}=N_{3}=0\right\}$. In particular,

$$
\left(\mathcal{B}_{\mathrm{II}} \cap \mathcal{K}\right) \cap U=\left\{x_{1}=x_{2}=0\right\} \cup\left\{x_{1}=z=0\right\} \cup\left\{x_{2}=z=0\right\} .
$$

(v) The right-hand side of (7) is unchanged if one replaces $x_{1}$ (resp. $x_{2}$ and $z$ ) by $-x_{1}$ (resp. by $-x_{2}$ and $\left.-z\right)$. Therefore, we may assume that

$$
\mathcal{B}^{+} \cap U=\left\{x_{1} \geq 0, x_{2} \geq 0, z \geq 0\right\} .
$$

(vi) On the one hand, according to item (i), the metric induced on the one-dimensional submanifold $\mathcal{K} \cap U$ by the riemannian metric $\left(d x_{1}\right)^{2}+\left(d x_{2}\right)^{2}+(d y)^{2}+(d z)^{2}$ is simply $(d y)^{2}$. On the other hand, the right-hand side of (7) is unchanged if one replaces $y$ by $\varphi(y)$ where $\varphi: \mathbb{R} \rightarrow \mathbb{R}$ is any diffeomorphism such that $\varphi(0)=0$. Therefore, up to replacing the coordinate $y$ by $\varphi(y)$ for some appropriate diffeomorphism $\varphi$, we may assume that the metrics induced on the piece of circle $\mathcal{K} \cap U$ by the riemannian metrics $\left(d x_{1}\right)^{2}+\left(d x_{2}\right)^{2}+(d y)^{2}+(d z)^{2}$ andby the riemannian metric $h=\left(d \Sigma_{1}\right)^{2}+\left(d \Sigma_{2}\right)^{2}+\left(d \Sigma_{3}\right)^{2}+\left(d N_{1}\right)^{2}+\left(d N_{2}\right)^{2}+\left(d N_{3}\right) 2$ coincide.

Proof of proposition 3.2. The derivative $D X_{\mathcal{B}}(p)$ has two negative, one zero, and one positive eigenvalue. According to proposition 3.1, the vector field $X_{\mathcal{B}}$ satisfies Sternberg's condition at $p$. Therefore, a crude application of Takens' theorem 2.2 implies that there exists a $C^{1}$ local coordinate system $\left(x_{1}, x_{2}, y, z\right)$ on a neighborhood $U$ of $p$ in $\mathcal{B}$, centered at $p$, such that :

$$
X_{\mathcal{B}}\left(x_{1}, x_{2}, y, z\right)=\sum_{i=1}^{2} \sum_{j=1} 2 a_{i, j}(y) x^{j} \frac{\partial}{\partial x^{i}}+\phi(y) \frac{\partial}{\partial y}+b(y) z \frac{\partial}{\partial z}
$$

for some real valued functions $\phi, a_{1,1}, a_{1,2}, a_{2,1}, a_{2,2}, b$ defined on a neighborhood of 0 in $\mathbb{R}$. Moreover, the eigenvalues of the matrix $\left(a_{i, j}(0)\right)$ are negative, and $b(0)$ is positive. Replacing $U$ by a smaller neighborhood of $p$ if necessary, we can assume that the three Taub points $T_{1}, T_{2}, T_{3}$ are not in $U$. 
Now, (8) implies that the curve $\left\{x_{1}=x_{2}=z=0\right\}$ is the only curve in $U$ containing the point $p$, invariant under the flow of $X_{\mathcal{B}}$, and such that $D X_{\mathcal{B}}(p)$ vanishes on the tangent space at $p$ of this curve. Hence, the curve $\left\{x_{1}=x_{2}=z=0\right\}$ has to be the intersection of the Kasner circle $\mathcal{K}$ with $U$. Since $X_{\mathcal{B}}$ vanishes on $\mathcal{K}$, it follows that $\phi=0$.

For $\zeta$ small enough, let $q_{\zeta}$ be the point of coordinates $\left(x_{1}, x_{2}, y, z\right)=(0,0, \zeta, 0)$. This is a point of the Kasner circle $\mathcal{K}$, which is not a Taub point. Hence, the derivative $D X\left(q_{\zeta}\right)$ has four distinct eigenvalues : two distinctive negative eigenvalues $\lambda_{1}^{s}(\zeta)<\lambda_{2}^{s}(\zeta)<0$, one zero eigenvalue associated to the direction of the Kasner circle, and one positive eigenvalue $\lambda^{u}(\zeta)>0$. The set $(y=\zeta)$ is a three-dimensional manifold, transversal to the Kasner circle. Looking at (8), we see that this three-dimensional submanifold is invariant under the flow of $X_{\mathcal{B}}$, and that the restriction of $X_{\mathcal{B}}$ to this invariant manifold is linear.This shows that $b(\zeta)=\lambda^{u}(\zeta)$, and that $\lambda_{1}^{s}(\zeta)$ and $\lambda_{2}^{s}(\zeta)$ are the eigenvalues of the matrix $\left(a_{i, j}(\zeta)\right)_{i, j=1,2}$. Since $\lambda_{1}^{s}(\zeta)$ and $\lambda_{2}^{s}(\zeta)$ are distinct, there exists a linear change of coordinates $\left(x_{1}, x_{2}, z\right) \rightarrow\left(\hat{x}_{1}, \hat{x}_{2}, z\right)$ on the submanifold $(y=\zeta)$, so that

$$
X_{\mathcal{B}}\left(\hat{x}_{1}, \hat{x}_{2}, \zeta, z\right)=\lambda_{1}^{s}(\zeta) \hat{x}_{1} \frac{\partial}{\partial \hat{x}_{1}}+\lambda_{2}^{s}(\zeta) \hat{x}_{2} \frac{\partial}{\partial \hat{x}_{2}}+\lambda^{u}(\zeta) z \frac{\partial}{\partial z} .
$$

Since eigenvalues and eigendirections of the point $q_{\zeta}$ depend in a smooth way of $\zeta$, one may perform the above change of coordinates simultaneously for every $\zeta$, and get a $C^{1}$ coordinate system $\left(\hat{x}_{1}, \hat{x}_{2}, y, z\right)$ defined on $U$, such that

$$
X_{\mathcal{B}}\left(\hat{x}_{1}, \hat{x}_{2}, y, z\right)=\lambda_{1}^{s}(y) \hat{x}_{1} \frac{\partial}{\partial \hat{x}_{1}}+\lambda_{2}^{s}(y) \hat{x}_{2} \frac{\partial}{\partial \hat{x}_{2}}+\lambda^{u}(y) z \frac{\partial}{\partial z} .
$$

The proposition is proven.

3.4. Choice of a linearizing coordinate system. From now on, for every point $p$ of the Kasner circle which is not preperiodic under the Kasner map, we fix a neighborhood $U^{p}$ of $p$ in $\mathcal{B}$, and a $C^{1}$ local coordinate system $\left(x_{1}^{p}, x_{2}^{p}, y^{p}, z^{p}\right)$ on $U^{p}$, centered at $p$, such that, in this coordinate system, the Wainwright-Hsu vector field $X_{\mathcal{B}}$ reads:

$$
X_{\mathcal{B}}\left(x_{1}^{p}, x_{2}^{p}, y^{p}, z^{p}\right)=\lambda_{1}^{s}\left(y^{p}\right) x_{1}^{p} \frac{\partial}{\partial x_{1}^{p}}+\lambda_{2}^{s}\left(y^{p}\right) x_{2}^{p} \frac{\partial}{\partial x_{2}^{p}}+\lambda^{u}\left(y^{p}\right) z^{p} \frac{\partial}{\partial z^{p}}
$$

with $\lambda_{1}^{s}\left(y^{p}\right)<\lambda_{2}^{s}\left(y^{p}\right)<0$ and $\lambda^{u}\left(y^{p}\right)>0$. According to item (ii) of remarks 3.3, the real numbers $\lambda_{1}^{s}\left(y^{p}\right), \lambda_{2}^{s}\left(y^{p}\right), \lambda^{u}\left(y^{p}\right)$ are the non-zero eigenvalues of the point $\left(0,0, y^{p}, 0\right)$. According to items (i), (iv), (v) of remarks 3.3, up to changing the sign of the coordinates $x_{1}^{p}, x_{2}^{p}$ and $z^{p}$, we may (and we will) assume that

$$
\begin{aligned}
\mathcal{K} \cap U^{p} & =\left\{x_{1}^{p}=x_{2}^{p}=z=0\right\} \\
\left(\mathcal{B}_{\mathrm{II}} \cup \mathcal{K}\right) \cap U^{p} & =\left\{x_{1}^{p}=x_{2}^{p}=0\right\} \cup\left\{x_{1}^{p}=z^{p}=0\right\} \cup\left\{x_{2}^{p}=z^{p}=0\right\}, \\
\mathcal{B}^{+} \cap U^{p} & =\left\{x_{1}^{p} \geq 0, x_{2}^{p} \geq 0, z^{p} \geq 0\right\}
\end{aligned}
$$

Note that the derivative $D X_{\mathcal{B}}(q)$ has three non-zero eigenvalues for every point $q \in \mathcal{K} \cap U^{p}$; this shows that the neighborhood $U^{p}$ is disjoint from the three special points. We will consider the riemannian metric $g^{p}$ on $U^{p}$ defined by

$$
g^{p}:=\left(d x_{1}^{p}\right)^{2}+\left(d x_{2}^{p}\right)^{2}+\left(d y^{p}\right)^{2}+\left(d z^{p}\right) 2 .
$$


According to item (vi) of remarks 3.3, up to replacing the coordinate $y^{p}$ by $\varphi\left(y^{p}\right)$ for some appropriate diffeomorphism $\varphi$, we may (and we will) assume that $g^{p}$ induces the same metric on the piece of Kasner circle $\mathcal{K} \cap U^{p}$ as the riemannian metric $h=\left(d \Sigma_{1}\right)^{2}+\left(d \Sigma_{2}\right)^{2}+\left(d \Sigma_{3}\right)^{2}+\left(d N_{1}\right)^{2}+\left(d N_{2}\right)^{2}+\left(d N_{3}\right)^{2}$.

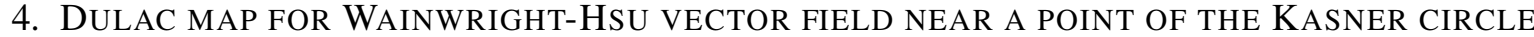 WHICH IS NOT PREPERIODIC UNDER THE KASNER MAP}

Let $p$ be a point of the Kasner circle which is not preperiodic for the Kasner map. The purpose of the present section is to analyze the behavior of the orbits of the Wainwright-Hsu vector field $X_{\mathcal{B}}$ close to $p$. More precisely, we want to consider an orbit of $X_{\mathcal{B}}$ which passes close to $p$, and to study the evolution of the distance from this orbit to the mixmaster attractor $\mathcal{A}=\mathcal{K} \cup \mathcal{B}_{\mathrm{II}}$, as well as the drift of this orbit in the direction tangent to the mixmaster attractor.

4.1. The flow of $X_{\mathcal{B}}$ inside $U^{p}$. We consider the neighborhood $U^{p}$, and the coordinate system $\left(x_{1}^{p}, x_{2}^{p}, y^{p}, z^{p}\right)$ defined in subsection 3.4. Using the expression (9), one can calculate explicitly the time tmap $X_{\mathcal{B}}^{t}$ of the flow of the Wainwright-Hsu vector field $X_{\mathcal{B}}$ in the $\left(x_{1}^{p}, x_{2}^{p}, y^{p}, z^{p}\right)$ coordinate system. It reads

$$
X_{\mathcal{B}}^{t}\left(x_{1}^{p}, x_{2}^{p}, y^{p}, z^{p}\right)=\left(x_{1}^{p} e^{\lambda_{1}^{s}\left(y^{p}\right) t}, x_{2}^{p} e^{\lambda_{2}^{s}\left(y^{p}\right) t}, y^{p}, z^{p} e^{\lambda^{u}\left(y^{p}\right) t}\right) .
$$

Of course, this expression is only valid as long as the orbit remains in the neighborhood $U^{p}$.

4.2. The box $V^{p}$. Now, we fix some constants $\alpha<\beta$ and $\gamma>0$, and we consider the subset $V^{p}$ of $U^{p} \cap \mathcal{B}^{+}$defined by:

$$
V^{p}=V^{p}(\alpha, \beta, \gamma)=\left\{0 \leq x_{1}^{p} \leq \gamma, 0 \leq x_{2}^{p} \leq \gamma, 0 \leq z^{p} \leq \gamma \text { and } \alpha \leq y^{p} \leq \beta\right\} .
$$

We assume that $\alpha, \beta, \gamma$ are small enough, so that $V^{p}$ is contained in the interior of $U^{p}$.

Remark 4.1. The set $V^{p}$ is a neighborhood of the point $p$ in $\mathcal{B}^{+}$if and only if $\alpha<0<\beta$. It is important to note that the results of the present section are valid even if $p$ is not in $V^{p}$.

In the $\left(x_{1}^{p}, x_{2}^{p}, y^{p}, z^{p}\right)$ coordinate system, the set $V^{p}$ is a 4-dimensional box, i.e. the cartesian product of four closed intervals. The boundary of $V^{p}$ is made of eight faces. Three of these eight faces will play an important role in the remainder of the paper:

$$
M_{1}^{p}:=V^{p} \cap\left\{x_{1}^{p}=\gamma\right\} \quad M_{2}^{p}:=V^{p} \cap\left\{x_{2}^{p}=\gamma\right\} \quad N^{p}:=V^{p} \cap\left\{z^{p}=\gamma\right\} .
$$

Looking at (9), we notice that $X_{\mathcal{B}}$ is transversal to $M_{1}^{p}, M_{2}^{p}$ and $N^{p}$, and is tangent to the five other faces of $V^{p}$. Moreover, we notice that $X_{\mathcal{B}}$ is pointing inward $V^{p}$ along $M_{1}^{p}$ and $M_{2}^{p}$; it is pointing outward $V^{p}$ along $N^{p}$. It follows that:

- an orbit of $X_{\mathcal{B}}$ can enter in $V^{p}$ by crossing either the face $M_{1}^{p}$ or by crossing the face $M_{2}^{p}$;

- an orbit of $X_{\mathcal{B}}$ can only exit $V^{p}$ by crossing the face $N^{p}$. 
4.3. Behavior of type II orbits. We will study the behavior of the orbits of $X_{\mathcal{B}}$ in $V^{p}$. First, we focus our attention on type II orbits. We want to understand which type II orbit intersect the hypersurfaces (with boundary and corners) $M_{1}^{p}, M_{2}^{p}$ and $N^{p}$. Recall that every type II orbit $\mathcal{O}$ of $X_{\mathcal{B}}$ is a heteroclinic orbit connecting a point $\alpha(\mathcal{O}) \in \mathcal{K} \backslash\left\{T_{2}, T_{2}, T_{3}\right\}$ to the point $\omega(\mathcal{O})=f(\alpha(\mathcal{O})) \in \mathcal{K} \backslash\left\{T_{2}, T_{2}, T_{3}\right\}$.

Proposition 4.2. Let $q$ be a point $\mathcal{B}_{I I}^{+}$, and denote by $\mathcal{O}$ the orbit of $q$. Let $\alpha(q)=\alpha(\mathcal{O})$ denote the unique $\alpha$-limit point of $\mathcal{O}$, and $\omega(q)=\omega(\mathcal{O})=f(\alpha(\mathcal{O}))$ denote the unique $\omega$-limit point of $\mathcal{O}$.

(1) The orbit $\mathcal{O}$ intersects the hypersurface $N^{p}$ if and only if the point $\alpha(q)$ is in $V^{p}$.

(2) The orbit of $\mathcal{O}$ intersects the hypersurface $M_{1}^{p} \cup M_{2}^{p}$ if and only if the point $\omega(q)$ is in $V^{p}$.

Proof. We prove the first statement; the second one follows from similar arguments. Of course, we will work in the $\left(x_{1}^{p}, x_{2}^{p}, y^{p}, z^{p}\right)$ coordinate system. According to (10), (11), (15) and (16),

$$
\begin{aligned}
\mathcal{K} \cap V^{p} & =\left\{x_{1}^{p}=x_{2}^{p}=0, \alpha \leq y^{p} \leq \beta, z^{p}=0\right\}, \\
\mathcal{B}_{\mathrm{II}} \cap N^{p} & =\left\{x_{1}^{p}=x_{2}^{p}=0, \alpha \leq y^{p} \leq \beta, z^{p}=\gamma\right\} .
\end{aligned}
$$

Suppose that the orbit $\mathcal{O}$ intersects the hypersurface $N^{p}$ at some point $\bar{q}=(0,0, \zeta, \gamma)$, with $\alpha \leq \zeta \leq \beta$. Then, according to (14), the past orbit of $\bar{q}$ is contained in $V^{p}$, and converges to the point $\alpha(q)=$ $(0,0, \zeta, 0) \in \mathcal{K} \cap V^{p}$. In particular, the point $\alpha(q)$ is in $V^{p}$. Conversely, suppose that the point $\alpha(q)$ is in $V^{p}$. Then $\alpha(q)=(0,0, \zeta, 0)$ for some $\zeta \in[\alpha, \beta]$. Using again (14), we see that the only orbit of $X_{\mathcal{B}}$ in $V^{p}$ converging towards the point $(0,0, \zeta, 0)$ as $t \rightarrow-\infty$ is the curve $t \mapsto\left(0,0, \zeta, e^{\lambda^{u}(\zeta) t}\right)$. Hence, the orbit $\mathcal{O}$ intersects the hypersurface $N^{p}$ at the point $\bar{q}=(0,0, \zeta, \gamma)$.

This proposition allows to define two maps

$$
\begin{aligned}
\alpha^{p}: \mathcal{B}_{\mathrm{II}}^{+} \cap N^{p} & \longrightarrow \mathcal{K} \cap V^{p} & \omega^{p}: \mathcal{B}_{\mathrm{II}}^{+} \cap\left(M_{1}^{p} \cup M_{2}^{p}\right) & \longrightarrow \mathcal{K} \cap V^{p} \\
q & \longmapsto \alpha(q) & & \longmapsto \omega(q)
\end{aligned}
$$

The map $\alpha^{p}$ is one-to-one (there is only one type II orbit in $\mathcal{B}^{+}$which "starts" at a given point of $\mathcal{K} \backslash\left\{T_{2}, T_{2}, T_{3}\right\}$ ), whereas the map $\omega^{p}$ is two-to-one (there are two type II orbits in $\mathcal{B}^{+}$which "arrive" at a given point of $\mathcal{K} \backslash\left\{T_{2}, T_{2}, T_{3}\right\}$ ). The restriction of $\omega^{p}$ to $\mathcal{B}_{\mathrm{II}}^{+} \cap M_{1}^{p}$ (resp. to $\mathcal{B}_{\mathrm{II}}^{+} \cap M_{2}^{p}$ ) is one-to-one.

Proposition 4.3. The maps $\alpha^{p}$ and $\omega^{p}$ are local isometries with respect to the metrics induced on $\mathcal{B}_{I I}^{+} \cap N^{p}, \mathcal{B}_{I I}^{+} \cap\left(M_{1}^{p} \cup M_{2}^{p}\right)$ and $\mathcal{K} \cap V^{p}$ by the riemannian metric $g^{p}=\left(d x_{1}^{p}\right)^{2}+\left(d x_{2}^{p}\right)^{2}+\left(d y^{p}\right)^{2}+\left(d z^{p}\right) 2$.

Proof. The proof of proposition 4.2 shows that, in the $\left(x_{1}^{p}, x_{2}^{p}, y^{p}, z^{p}\right)$ coordinate system, the map $\alpha^{p}$ reads $\alpha^{p}\left(0,0, y^{p}, \gamma\right)=\left(0,0, y^{p}, 0\right)$. Similarly, the map $\omega^{p}$ reads $\omega^{p}\left(\gamma, 0, y^{p}, 0\right)=\left(0,0, y^{p}, 0\right)$.

4.4. The Dulac map $\Delta_{1}^{p}: M_{1}^{p} \rightarrow N^{p}$. Now, we want to study the behavior of arbitrary orbits of $X_{\mathcal{B}}$ which enter in $V^{p}$. Let $q$ be a point on the face $M_{1}^{p}$. Denote by $\left(\gamma, x_{2}^{p}, y^{p}, z^{p}\right)$ the coordinates of $q$. If the $z^{p}>0$ (which is typically the case if $q \in \mathcal{B}_{\text {IX }}$ ), then (14) shows that the forward orbit of $q$ will eventually exit $V^{p}$ by crossing the face $N^{p}$. If $z^{p}=0$ (which is typically the case if $q \in \mathcal{B}_{\text {II }}$ ), then (14) shows that the forward orbit of $q$ will remain in $V^{p}$ forever. It will converge towards the point $\omega(q) \in \mathcal{K} \cap V^{p}$. According to proposition 4.2, the heteroclinic orbit $\mathcal{O}_{\omega(q), f(\omega(q))}$ will eventually exit $V^{p}$, by crossing the face $N^{p}$. So, we may define a map $\Delta_{1}^{p}: M_{1}^{p} \longrightarrow N^{p}$ as follows :

- if $z^{p}>0$ then $\Delta_{1}^{p}(q)$ is the first intersection point of the orbit of $q$ with the hypersurface $N^{p}$; 
- if $z^{p}=0$ then $\Delta_{1}^{p}(q)$ is the first (and unique) intersection point of the type II heteroclinic orbit $\mathcal{O}_{\omega(q), f(\omega(q))}$ with the hypersurface $N^{p}$.

We call $\Delta_{1}^{p}$ a Dulac map since it is the exact analog, in our situation, of the classical Dulac maps used to study planar vector fields. Formula (14) show that, in the $\left(x_{1}^{p}, x_{2}^{p}, y^{p}, z^{p}\right)$ coordinate system, the map $\Delta_{1}^{p}$ reads :

$$
\begin{aligned}
\Delta_{1}^{p}\left(\gamma, x_{2}^{p}, y^{p}, z^{p}\right) & =\left(\gamma \cdot\left(\frac{z^{p}}{\gamma}\right)^{-\frac{\lambda_{1}^{s}\left(y^{p}\right)}{\lambda^{u}\left(y^{p}\right)}}, x_{2}^{p} \cdot\left(\frac{z^{p}}{\gamma}\right)^{-\frac{\lambda_{2}^{s}\left(y^{p}\right)}{\lambda^{u}\left(y^{p}\right)}}, y^{p}, \gamma\right) \text { if } z^{p}>0 \\
\Delta^{p}\left(\gamma, x_{2}^{p}, y^{p}, 0\right) & =\left(0,0, y^{p}, \gamma\right)
\end{aligned}
$$

Remark 4.4. Given a point $q$ in $M_{1}^{p}$ such that $z^{p}(q)>0$, one can consider the exit time of $q$, that is the real number $t(q)$ such that $\Delta_{1}^{p}(q)=X^{t(q)}(q)$. Using (14), it is easy to see

$$
t\left(\gamma, x_{2}^{p}, y^{p}, z^{p}\right)=-\frac{1}{\lambda^{u}\left(y^{p}\right)} \log \left(\frac{z^{p}}{\gamma}\right)
$$

For every $q \in M_{1}^{p}$, we decompose $T_{q} M^{p}$ as a direct sum of two linear subspaces $F_{q}^{s} \oplus F_{q}^{u}$ where

$$
\begin{aligned}
F_{q}^{s} & :=\mathbb{R} \cdot \frac{\partial}{\partial x_{2}^{p}}(q) \oplus \mathbb{R} \cdot \frac{\partial}{\partial z^{p}}(q) \\
F_{q}^{u} & :=\mathbb{R} \cdot \frac{\partial}{\partial y^{p}}(q) .
\end{aligned}
$$

Similarly, for every $r \in N^{p}$, we decompose $T_{r} N^{p}$ as a direct sum of two linear subspaces $G_{r}^{s} \oplus F_{r}^{u}$ where

$$
\begin{aligned}
G_{r}^{s} & :=\mathbb{R} \cdot \frac{\partial}{\partial x_{1}^{p}}(r) \oplus \mathbb{R} \cdot \frac{\partial}{\partial x_{2}^{p}}(r) \\
G_{r}^{u} & :=\mathbb{R} \cdot \frac{\partial}{\partial y^{p}}(r) .
\end{aligned}
$$

We can now state the properties of the Dulac map $\Delta_{1}^{p}$ which will be the core of our proof of theorem 1.3:

Proposition 4.5. The Dulac map $\Delta_{1}^{p}: M_{1}^{p} \rightarrow N^{p}$ is $C^{1}$. Moreover, for every point $q \in M_{1}^{p} \cap\left\{z^{p}=0\right\}$, the derivative of the map $\Delta_{1}^{p}$ at $q$ satisfies:

- $D \Delta_{1}^{p}(q) \cdot v=0$ for every vector $v \in F_{q}^{s}$;

- $D \Delta_{1}^{p}(q)$ maps $F_{q}^{u}$ on $G_{\Delta_{1}^{p}(q)}^{u}$, and $\left\|D \Delta_{1}^{p}(q) . v\right\|_{g^{p}}=\|v\|_{g^{p}}$ for every vector $v \in F_{q}^{u}$.

This proposition roughly says the following: when an orbit of $X_{\mathcal{B}}$ passes close to the point $p \in \mathcal{K}$, the distance from this orbit to the mixmaster attractor $\mathcal{A}=\mathcal{K} \cup \mathcal{B}_{\mathrm{II}}$ is contracted super-linearly (this distance is measured by the coordinates $x_{1}^{p}, x_{2}^{p}$ and $z^{p}$ ), whereas there is no drift in the direction tangent to the attractor (this drift is measured by the coordinate $y^{p}$ ). The key point of the proof of proposition 4.5 is the following elementary observation:

Lemma 4.6. For every $y^{p}$, we have $\left|\frac{\lambda_{1}^{s}\left(y^{p}\right)}{\lambda^{u}\left(y^{p}\right)}\right|>1$ and $\left|\frac{\lambda_{2}^{s}\left(y^{p}\right)}{\lambda^{u}\left(y^{p}\right)}\right|>1$. 
This lemma says that, at every point $\bar{q}$ of $\mathcal{K} \backslash\left\{T_{2}, T_{2}, T_{3}\right\}$, the positive eigenvalue of the derivative $D X(\bar{q})$ is dominated by the contracting eigenvalues.

Proof of lemma 4.6. Fix $y^{p}$, and denote by $q$ be the point of coordinates $\left(0,0, y^{p}, 0\right)$ in the $\left(x_{1}^{p}, x_{2}^{p}, y^{p}, z^{p}\right)$ coordinate system. Denote by $\left(\Sigma_{1}, \Sigma_{2}, \Sigma_{3}, 0,0,0\right)$ the coordinates of $q$ in the Wainwright-Hsu coordinate system, and by $u$ the Kasner parameter of $q$. The real numbers $\lambda_{1}^{s}\left(y^{p}\right), \lambda_{2}^{s}\left(y^{p}\right), \lambda^{u}\left(y^{p}\right)$ are the three nonzero eigenvalues the derivative $D X_{\mathcal{B}}(q)$. Hence, these numbers are equal up to permutation to $-\left(2+\Sigma_{1}\right)$, $-\left(2+\Sigma_{2}\right),-\left(2+\Sigma_{3}\right)$. Using (5) and the inequalities $\lambda_{1}^{s}\left(y^{p}\right)<\lambda_{2}^{s}\left(y^{p}\right)<0<\lambda^{u}\left(y^{p}\right)$, we deduce that

$$
\left(\lambda_{1}^{s}\left(y^{p}\right), \lambda_{2}^{s}\left(y^{p}\right), \lambda^{u}\left(y^{p}\right)\right)=\left(\frac{-6 u(1+u)}{1+u+u^{2}}, \frac{-6(1+u)}{1+u+u^{2}}, \frac{6 u}{1+u+u^{2}}\right) .
$$

The lemma follows since $\frac{1+u}{u}>1$ and $(1+u)>1$ for every $u \geq 1$.

Proof of proposition 4.5. The fact that $\Delta_{1}^{p}$ is $C^{1}$ (and even analytical) in restriction to $M_{1}^{p} \cap\left\{z^{p}>0\right\}$ is an immediate consequence of formula (17). The fact that $\Delta_{1}^{p}$ is $C^{1}$ on $M_{1}^{p} \cap\left\{z^{p}=0\right\}$ follows from (17), (18) and lemma 4.6. The same ingredients actually show that, for every point $q \in M^{p} \cap\left\{z^{p}=\right.$ $0\}$,

$$
D \Delta_{1}^{p}(q) \cdot \frac{\partial}{\partial x_{2}^{p}}(q)=D \Delta_{1}^{p}(q) \cdot \frac{\partial}{\partial z^{p}}(q)=0 \quad \text { and } D \Delta_{1}^{p}(q) \cdot \frac{\partial}{\partial y^{p}}(q)=\frac{\partial}{\partial y^{p}}\left(\Delta_{1}^{p}(q)\right) .
$$

The proposition follows.

Remark 4.7. We do not know if $\Delta_{1}^{p}$ is $C^{1+\varepsilon}$ for any given $\varepsilon>0$, unless we have some a priori lower bounds for the distance between the ratios $\frac{\lambda_{1}^{s}\left(y^{p}\right)}{\lambda^{u}\left(y^{p}\right)}$ and $\frac{\lambda_{2}^{s}\left(y^{p}\right)}{\lambda^{u}\left(y^{p}\right)}$ and 1. This is the reason why, in the statement of theorem 1.3, we cannot guarantee that the stable manifold $W^{s}(q)$ contains a $C^{1+\varepsilon}$-embedded disc for any given $\varepsilon$. Actually such an $\varepsilon$ exists for every $q$ but it does depend on $q$, and tends to 0 if $q$ approaches one of the three special points tends to 0 .

4.5. The Dulac map $\Delta_{2}^{p}: M_{2}^{p} \rightarrow N^{p}$. The coordinates $x_{1}^{p}$ and $x_{2}^{p}$ play similar roles in the expression of the vector field $X_{\mathcal{B}}$ and in the definition of $V^{p}$. So, we have to consider a second Dulac map $\Delta_{2}^{p}: M_{2}^{p} \longrightarrow$ $N^{p}$ defined as follows. Let $q$ be a point on the face $M_{1}^{p}$. Denote by $\left(\gamma, x_{2}^{p}, y^{p}, z^{p}\right)$ the coordinates of $q$.

- if $z^{p}(q)>0$ then $\Delta_{2}^{p}(q)$ is the first intersection point of the orbit of $q$ with the hypersurface $N^{p}$;

- if $z^{p}(q)=0$ then $\Delta_{2}^{p}(q)$ is the first (and unique) intersection point of the type II heteroclinic orbit $\mathcal{O}_{\omega(q), f(\omega(q))}$ with the hypersurface $N^{p}$.

In the $\left(x_{1}^{p}, x_{2}^{p}, y^{p}, z^{p}\right)$ coordinate system, the Dulac map $\Delta_{2}^{p}$ reads:

$$
\begin{aligned}
\Delta_{2}^{p}\left(x_{1}^{p}, \gamma, y^{p}, z^{p}\right) & =\left(x_{1}^{p} \cdot\left(\frac{z^{p}}{\gamma}\right)^{-\frac{\lambda_{1}^{s}\left(y^{p}\right)}{\lambda^{u}\left(y^{p}\right)}}, \gamma \cdot\left(\frac{z^{p}}{\gamma}\right)^{-\frac{\lambda_{2}^{s}\left(y^{p}\right)}{\lambda^{u}\left(y^{p}\right)}}, y^{p}, \gamma\right) \text { if } z^{p}>0 \\
\Delta_{2}^{p}\left(x_{1}^{p}, \gamma, y^{p}, 0\right) & =\left(0,0, y^{p}, \gamma\right)
\end{aligned}
$$

For every $q \in M_{2}^{p}$, we will write $T_{q} M_{2}^{p}$ as a direct sum of two linear subspaces $F_{q}^{s} \oplus F_{q}^{u}$ where

$$
\begin{aligned}
F_{q}^{s} & :=\mathbb{R} \cdot \frac{\partial}{\partial x_{1}^{p}}(q) \oplus \mathbb{R} \cdot \frac{\partial}{\partial z^{p}}(q) \\
F_{q}^{u} & :=\mathbb{R} \cdot \frac{\partial}{\partial y^{p}}(q) .
\end{aligned}
$$


Then, we can summarize the key properties of the map $\Delta_{2}^{p}$ as follow :

Proposition 4.8. The map $\Delta_{2}^{p}: M_{2}^{p} \rightarrow N^{p}$ is $C^{1}$. Moreover, for every $q \in M_{2}^{p} \cap\left\{z^{p}=0\right\}$, the derivative of of the map $\Delta_{2}^{p}$ at $q$ satisfies :

- $D \Delta_{2}^{p}(q) . v=0$ for every vector $v \in F_{q}^{s}$;

- $D \Delta_{2}^{p}(q)$ maps $F_{q}^{u}$ on $G_{\Delta_{2}^{p}(q)}^{u}$, and $\left\|D \Delta_{2}^{p}(q) . v\right\|_{g^{p}}=\|v\|_{g^{p}}$ for every vector $v$ in $F_{q}^{u}$.

\section{CONSTRUCTION OF A "PoincARÉ MAP" ASSOCIATED TO A ClOSED FORWARD-INVARIANT APERIODIC SET OF THE KASNER CIRCLE}

From now on until the end of section 7, we consider a closed forward-invariant aperiodic subset $C$ of the Kasner circle $\mathcal{K}$.

Observe that $C$ is necessarily totally discontinuous. Indeed the points of $\mathcal{K}$ that are preperiodic under the Kasner map $f$ are dense in $\mathcal{K}$ (this can be proved in several different ways; this follows for example the equivalence $2 \Leftrightarrow 3$ of proposition 3.1). We shall denote by $\widehat{C}$ the union of $C$ and all the type II orbits connecting two points of $C$ :

$$
\widehat{C}:=C \cup \bigcup_{q \in C} \mathcal{O}_{q, f(q)} \cdot
$$

We want to prove that, for every point $q \in C$, the stable manifold $W^{s}(q)$ contains a 3-dimensional disc (see definition 1.2 and theorem 1.3). To this end, we will consider a kind of "Poincaré section" for $\widehat{C}$, and study the Poincaré map associated with this section ${ }^{1}$.

For every $p \in C$, we consider a neighborhood $U^{p}$ of $p$, and a local coordinate system $\left(x_{1}^{p}, x_{2}^{p}, y^{p}, z^{p}\right)$ on $U^{p}$, as in the previous section. For each $p \in C$, we choose $\alpha_{0}^{p}<0<\beta_{0}^{p}$ and $\gamma_{0}^{p}>0$ small enough, so that

$$
V_{0}^{p}:=V^{p}\left(\alpha_{0}^{p}, \beta_{0}^{p}, \gamma_{0}^{p}\right)=\left\{0 \leq x_{1}^{p} \leq \gamma_{0}^{p}, 0 \leq x_{2}^{p} \leq \gamma_{0}^{p}, 0 \leq z^{p} \leq \gamma_{0}^{p} \text { and } \alpha_{0}^{p} \leq y^{p} \leq \beta_{0}^{p}\right\} .
$$

is contained in the interior of $U^{p}$. Up to slightly modifying $\alpha_{0}^{p}, \beta_{0}^{p}, \gamma_{0}^{p}$ we can assume that the boundary of $V_{0}^{p}$ is disjoint from $C$ (i.e that the points of coordinates $(0,0, \alpha, 0)$ and $(0,0, \beta, 0)$ in the $\left(x_{1}^{p}, x_{2}^{p}, y^{p}, z^{p}\right)$ coordinate system are not in $C$ ): this is possible since $C$ is totally discontinuous. Observe that $V_{0}^{p}$ is a neighborhood of $p$, since $\alpha_{0}^{p}<0<\beta_{0}^{p}$ (see remark 4.1).

Since $C$ is compact, one can find a finite number of points $p_{1}, \ldots, p_{n} \in C$ such that the neighborhoods $V_{0}^{p_{1}}, \ldots, V_{0}^{p_{n}}$ cover $C$. Now, we modify these neighborhoods in order to make them pairwise disjoint:

- we set $\left(\alpha^{p_{1}}, \beta^{p_{1}}, \gamma^{p_{1}}\right):=\left(\alpha_{0}^{p_{1}}, \beta_{0}^{p_{1}}, \gamma_{0}^{p_{1}}\right)$, and $V^{p_{1}}:=V^{p_{1}}\left(\alpha^{p_{1}}, \beta^{p_{1}}, \gamma^{p_{1}}\right)=V_{0}^{p_{1}}$;

- then, we can find some constants $\alpha_{2}, \beta_{2}, \gamma_{2}$ such that $V^{p_{2}}:=V^{p_{2}}\left(\alpha_{2}, \beta_{2}, \gamma_{2}\right)$ is contained in $V_{0}^{p_{2}} \backslash V^{p_{1}}$, and such that $C \cap\left(V_{0}^{p_{2}} \backslash V^{p_{1}}\right)$ is contained in the interior of $V^{p_{2}}$;

- then, we can find some constants $\alpha_{3}, \beta_{3}, \gamma_{3}$ such that $V^{p_{3}}:=V^{p_{3}}\left(\alpha_{3}, \beta_{3}, \gamma_{3}\right)$ is contained in $V_{0}^{p_{3}} \backslash\left(V^{p_{1}} \cup V^{p_{2}}\right)$, and such that $C \cap\left(V_{0}^{p_{3}} \backslash\left(V^{p_{1}} \cup V^{p_{2}}\right)\right)$ is contained in the interior of $V^{p_{3}}$;

- etc.

\footnotetext{
${ }^{1}$ The set $\widehat{C}$ cannot admit a true Poincaré section, since it contains some singularities of $X_{\mathcal{B}}$ (namely, the points of $C$ ). Nevertheless, we will consider a hypersurface $N$ such that every type II orbit in $\widehat{C}$ intersects $N$ transversally. The hypersurface $N$ will play the role of a Poincaré section.
} 
At the end of this process, we get $n$ pairwise disjoint domains $V^{p_{1}}, \ldots, V^{p_{n}}$, such that $C$ is contained in the interior of $V^{p_{1}} \cup \cdots \cup V^{p_{n}}$. For each $i, V^{p_{i}}$ is contained in the interior of $U^{p_{i}}$, and there are some constants $\alpha_{i}, \beta_{i}, \gamma_{i}$ such that $V^{p_{i}}=V^{p_{i}}\left(\alpha_{i}, \beta_{i}, \gamma_{i}\right)$. Hence, the result of section 4 apply to $V^{p_{i}}$. It may happen that, for some $i$, the point $p_{i}$ is not in $V^{p_{i}}$ (i.e. that $\alpha_{i}$ or $\beta_{i}$ is non-positive), but we do not care.

Now, we denote

$$
\begin{aligned}
V^{C} & :=V^{p_{1}} \sqcup \cdots \sqcup V^{p_{n}}, \\
M_{1}^{C} & :=M_{1}^{p_{1}} \sqcup \cdots \sqcup M_{1}^{p_{n}}, \\
M_{2}^{C} & :=M_{2}^{p_{1}} \sqcup \cdots \sqcup M_{2}^{p_{n}}, \\
M^{C} & :=M_{1}^{C} \cup M_{2}^{C} \\
N^{C} & :=N^{p_{1}} \sqcup \cdots \sqcup N^{p_{n}} .
\end{aligned}
$$

Then $V^{C}$ is a neighborhood of $C$ in $\mathcal{B}^{+}$. The hypersurfaces $M_{1}^{C}, M_{2}^{C}$ and $N^{C}$ are transverse to $X_{\mathcal{B}}$. An orbit of $X_{\mathcal{B}}$ can only enter in $V^{C}$ by crossing $M^{C}=M_{1}^{C} \cup M_{2}^{C}$, and can only exit $V^{C}$ by crossing $N^{C}$. Moreover, according to proposition 4.2, we have the following important properties :

Proposition 5.1. Every type II orbit whose $\omega$-limit point is in C intersects $M^{C}$. Every type II orbit whose $\alpha$-limit point is in $C$ intersects $N^{C}$.

We will see $M^{C}$ as a kind of "Poincaré section" for $\widehat{C}$. Let us define the "Poincaré map" $\Phi$ associated to this section. First, we consider the "Dulac map"

$$
\Delta^{C}: M^{C} \rightarrow N^{C}
$$

defined by $\Delta_{\mid M_{1}^{p_{i}}}^{C}=\Delta_{1}^{p_{i}}$ and $\Delta_{\mid M_{2}^{p_{i}}}^{C}=\Delta_{2}^{p_{i}}$. For $q \in M^{C}$ :

- if the forward orbit of $q$ exits $V^{C}$ by crossing $N^{C}$ (which is typically the case if $q \in \mathcal{B}_{\text {IX }}$ ), then $\Delta^{C}(q)$ is by definition the first intersection point of the orbit of $q$ with the hypersurface $N^{C}$;

- if the forward orbit of $q$ remains in $V^{C}$ forever (which is typically the case for every $q \in \mathcal{B}_{\mathrm{II}}$ ), then $\Delta^{C}(q)$ is the first intersection point of the type II heteroclinic orbit $\mathcal{O}_{\omega(q), f(\omega(q))}$ with the hypersurface $N^{C}$.

Now, we consider the "transition map"

$$
\Theta^{C}: N^{C} \rightarrow M^{C}
$$

partially defined as follows. Given a point $q$ in $N^{C}$, if the forward orbit of $q$ re-enters in $V^{C}$, then $\Theta^{C}(q)$ is the first point of this forward orbit of $q$ which is in $V^{C}$ (this point is automatically on the hypersurface $\left.M^{C}\right)$; otherwise $\Theta^{C}(q)$ is not defined. The "Poincare map" $\Phi^{C}$ associated with the section $M^{C}$ is by definition the product of the "Dulac map" $\Delta^{C}$ and the "transition map" $\Theta^{C}$ :

$$
\Phi^{C}:=\Theta^{C} \circ \Delta^{C}: M^{C} \rightarrow M^{C} .
$$

In the next section, we will study the dynamics of the Poincare map $\Theta^{C}$. For this purpose, we will use a riemannian metric on $g^{C}$ on $\mathcal{B}$ such that, for $i=1, \ldots, n$,

$$
g_{\mid V_{i}}^{C}=g^{p_{i}}=\left(d x_{1}^{p_{i}}\right)^{2}+\left(d x_{2}^{p_{i}}\right)^{2}+\left(d y^{p_{i}}\right)^{2}+\left(d z^{p_{i}}\right)^{2} .
$$




\section{Stable MANifolds For the Poincaré map ASSOCIATED to A ClOSED FORWARD-INVARIANT APERIODIC SET OF THE KASNER CIRCLE}

The purpose of this section is to prove that, for every point $q \in \widehat{C} \cap M^{C}$, the stable manifold of $q$ for the "Poincaré map" $\Phi^{C}$ contains a two-dimensional disc. To this end, we will prove that $\widehat{C} \cap M^{C}$ is a hyperbolic set for the map $\Phi^{C}$, and we will use a classical result on stable manifolds for hyperbolic sets.

Definition 6.1. Let $(M, g)$ be a riemannian manifold and $\Phi: M \rightarrow M$ be a $C^{1}$ map. A hyperbolic set for the map $\Phi$ is a compact $\Phi$-invariant subset $C$ of $M$ such that, for every $q \in C$, there is splitting $T_{q} M=F_{q}^{s} \oplus F_{q}^{u}$ which depends continuously on $q$ and such that, for some constant $\mu \in(0,1)$ and nu $>1$ :

$$
\begin{gathered}
D \Phi(q) . F_{q}^{s} \subset F_{\Phi(q)}^{s} \text { and }\|D \Phi(x) \cdot v\| \leq \mu\|v\| \text { for every } q \in C \text { and } v \in F_{q}^{s} \\
D \Phi(q) . F_{q}^{u}=F_{\Phi(q)}^{u} \text { and }\|D \Phi(q) \cdot v\| \geq \nu\|v\| \text { for every } q \in C \text { and } v \in F_{q}^{u} .
\end{gathered}
$$

The dimension of the vector space $F_{q}^{s}$ is called the index of $C$. The constant $\mu$ is called a contraction rate of $\Phi$ on $C$.

Theorem 6.2. (see e.g. [6, page 167]) Let $\Phi: M \rightarrow M$ be a $C^{1}$ map of a manifold $M$, and $C$ be a compact subset of $M$ which is a hyperbolic of index s for the map $\Phi$. Then, for every $\varepsilon$ small enough, for every $q \in C$, the set

$$
W_{\varepsilon}^{s}(\Phi, q):=\left\{r \in M \mid \operatorname{dist}\left(\Phi^{n}(r), \Phi^{n}(r)\right) \leq \varepsilon \text { for every } n \geq 0\right\}
$$

is a $C^{1}$ embedded s-dimensional disc, tangent to $F_{q}^{s}$ at $q$, depending continuously on $q$ (for the $C^{1}$ topology on the space of embeddings). Moreover, if $\mu$ is a contraction constant for $\Phi$ on $C$, then there exists a constant $\kappa$ such that, for every $\varepsilon$ small enough, for every $q \in C$ and every $r \in W_{\varepsilon}^{s}(\Phi, q)$,

$$
\operatorname{dist}_{g}\left(\Phi^{n}(r), \Phi^{n}(q)\right) \leq \kappa \mu^{n} .
$$

We want to apply this theorem to the Poincare map $\Phi^{C}: M^{C} \rightarrow M^{C}$. So we need to prove that $\widehat{C} \cap M^{C}$ is a hyperbolic set for $\Phi^{C}$. Recall that $M^{C}=M_{1}^{C} \cup M_{2}^{C}$ where $M_{1}^{C}=M_{1}^{p_{1}} \sqcup \cdots \sqcup M_{2}^{p_{n}}$ and $M_{2}^{C}=M_{2}^{p_{1}} \sqcup \cdots \sqcup M_{2}^{p_{n}}$. For every $q \in M^{C}$, we have already defined a splitting $T_{q} M^{C}=F_{q}^{s} \oplus F_{q}^{u}$ in section 4 (recall that $M^{C}=\left(M_{1}^{p_{1}} \cup \cdots \cup M_{1}^{p_{n}}\right) \cup\left(M_{2}^{p_{1}} \cup \cdots \cup M_{2}^{p_{n}}\right)$ and observe that $q$ is not in $\left.\left(M_{1}^{p_{1}} \cup \cdots \cup M_{1}^{p_{n}}\right) \cap\left(M_{2}^{p_{1}} \cup \cdots \cup M_{2}^{p_{n}}\right)\right)$. It remains to prove that $\Phi^{C}$ satisfies (27) and (28) with respect to these splitting. For this purpose, we will use the decomposition of $\Phi^{C}$ as a product :

$$
\Phi^{C}=\Theta^{C} \circ \Delta^{C} \text {. }
$$

The behavior of the derivative of "Dulac map" $\Delta^{C}$ was already studied in section 4 ; more precisely, we can rephrase propositions 4.5 and 4.8 as follows :

Proposition 6.3. The map $\Delta^{C}: M^{C} \rightarrow N^{C}$ is $C^{1}$. Moreover, for every $q \in \widehat{C} \cap M^{C}$, the derivative of $D \Delta^{C}(q): T_{q} M \rightarrow T_{\Delta^{C}(q)} N$ of the map $\Phi$ at $q$ satisfies :

- $D \Delta^{C}(q) \cdot v=0$ for every vector $v \in F_{q}^{s}$;

- $D \Delta^{C}(q)$ maps $F_{q}^{u}$ on $G_{\Delta^{C}(q)}^{u}$, and $\left\|D \Delta^{C}(q) \cdot v\right\|_{g_{p}}=\|v\|_{g_{p}}$ for every vector $v$ in $F_{q}^{u}$.

It remains to study the behavior of the derivative of the "transition map" $\Theta^{C}: N^{C} \rightarrow M^{C}$. We recall that $\Theta^{C}(q)$ is well-defined only if the forward orbit of $q$ intersects $M^{C}$. So our first task is to show that $\Theta^{C}$ is well-defined at least on a neighborhood of $\widehat{C} \cap N^{C}$ in $N^{C}$. 
Proposition 6.4. There exists a neighborhood $\mathcal{V}$ of $\widehat{C} \cap N^{C}$ in $N^{C}$, such that, for every $q \in \mathcal{V}$, the orbit of $q$ intersects $M^{C}$ after some time $t(q)$ which depends in a $C^{1}$ way on $q$. The map $\Theta^{C}$ is welldefined and $C^{1}$ on $\mathcal{V}$. Moreover, there exists $\nu>1$ such that, for every $q \in \widehat{C} \cap N^{C}$, the derivative $D \Theta^{C}(q): T_{q} N^{C} \rightarrow T_{\Theta^{C}(q)} M^{C}$ satisfies

- $D \Theta^{C}(q) \cdot G_{q}^{u}=F_{\Theta^{C}(q)}^{u}$ and $\left\|D \Theta^{C}(q) \cdot v\right\|_{g} \geq \nu\|v\|_{g}$ for every $v \in G_{q}^{u}$.

Proof. Consider a point $q \in \widehat{C} \cap N^{C}$. By proposition 5.1, the orbit of $q$ intersects $M^{C}$ at some point $r \in \widehat{C} \cap M^{C}$. Now, recall that :

- $N, M_{1}^{C}, M_{2}^{C}$ are $C^{1}$ hypersurfaces with boundary that are transversal to the orbits of $X_{\mathcal{B}}$;

- $V$ was chosen so that $C$ is contained in the interior of $V$. This implies that $\widehat{C}$ does not intersect neither the boundary of the hypersurface $N$, nor the boundary of hypersurface $M_{1}^{C}$ and $M_{2}^{C}$. It follows that $\widehat{C}$ does not intersect $M_{1}^{C} \cap M_{2}^{C}$. Hence, $q$ is in the interior of $N$, and $r$ is in the interior of $M_{1}^{C}$ or $M_{2}^{C}$.

This implies the existence of a neighborhood $\mathcal{V}_{q}$ of $q$ in $N$ such that, for every $q^{\prime} \in \mathcal{V}$, the forward orbit of $q^{\prime}$ intersects $M^{C}$ after some time $t\left(q^{\prime}\right)$ which depends in a $C^{1}$ way on $q^{\prime}$. By definition of $\Theta$, for every $q^{\prime} \in \mathcal{V}_{q}$, we have $\Theta\left(q^{\prime}\right):=X_{\mathcal{B}}^{t\left(q^{\prime}\right)}\left(q^{\prime}\right)$. In particular, $\Theta$ is well-defined and $C^{1}$ on $\mathcal{V}_{q}$. This proves the two first statements of the proposition.

Since $\mathcal{B}_{\text {II }}$ is invariant under the flow of $X_{\mathcal{B}}$, the map $\Theta^{C}$ maps $\mathcal{B}_{\mathrm{II}} \cap N^{C}$ on $\mathcal{B}_{\mathrm{II}} \cap M^{C}$. Now, observe that, for every $q \in \mathcal{B}_{\mathrm{II}} \cap N^{C}$, the direction $G_{q}^{u}$ is nothing but the tangent space of $\mathcal{B}_{\mathrm{II}} \cap N^{C}$ at $q$, and the direction $F_{\Theta^{C}(q)}^{u}$ is nothing but the tangent space of $\mathcal{B}_{\mathrm{II}} \cap M^{C}$ at $\Theta^{C}(q)$. This shows that $d \Theta^{C}(q)$ maps $G_{g}^{u}$ on $F_{\Theta^{C}(q)}^{u}$ for every $q \in \widehat{C} \cap N^{C}$.

We are left to prove the existence of a constant $\nu>1$ such that $\left\|D \Theta^{C}(q) \cdot v\right\|_{g} \geq \nu\|v\|_{g}$ for every $q \in \widehat{C} \cap N^{C}$ and every $v \in G_{q}^{u}$. For this purpose, we will use the maps

$$
\alpha: \mathcal{B}_{\mathrm{II}} \cap N^{C} \longrightarrow \mathcal{K} \cap V^{C} \quad \text { and } \quad \omega: \mathcal{B}_{\mathrm{II}} \cap M^{C} \longrightarrow \mathcal{K} \cap V^{C} .
$$

We recall that $\alpha$ maps a point $r \in \mathcal{B}_{\mathrm{II}} \cap N^{C}$ to the $\alpha$-limit point of the orbit of $r$, and that $\omega$ maps a point $s \in \mathcal{B}_{\mathrm{II}} \cap M^{C}$ to the $\omega$-limit point of the orbit of $s$ (see section 4). We also recall that $\alpha$ is a $C^{1}$ local isometry for the metrics induced by $g$ on $\mathcal{B}_{\mathrm{II}} \cap N^{C}$ and $\mathcal{K} \cap V^{C}$, and that $\omega$ is a $C^{1}$ local isometry for the metrics induced by $g$ on $\mathcal{B}_{\mathrm{II}} \cap M^{C}$ and $\mathcal{K} \cap V^{C}$ (proposition 4.3). Finally, we observe that, for $r \in \mathcal{B}_{\mathrm{II}} \cap N^{C}$,

$$
\omega\left(\Theta^{C}(r)\right)=\omega(r)=f(\alpha(r)) .
$$

The first equality is due to the fact that $\Theta^{C}(r)$ and $r$ are on the same orbit ; the second one is an immediate consequence of the definition of the Kasner map $f$. This shows that the last statement of proposition 6.4 is equivalent to the following statement about the Kasner map : there exists a constant $\nu>1$ such that, for every $p \in C$ and every $v \in T_{p} \mathcal{K}$, one has $\|D f(p) . v\|_{g} \geq \nu .|v|_{g}$.

This last statement is an immediate consequence of the elementary properties of the Kasner map, and of our choice of the riemannian metric $g^{C}$. Indeed, the riemannian metric $g^{C}$ was chosen so that it induces the same metric on $\mathcal{K} \cap V^{C}$ as the euclidean metric $h=\left(d \Sigma_{1}\right)^{2}+\left(d \Sigma_{2}\right)^{2}+\left(d \Sigma_{3}\right)^{2}+\left(d N_{1}\right)^{2}+$ $\left(d N_{2}\right)^{2}+\left(d N_{3}\right)^{2}$ (see the end of subsection 3.4 and the end of section 5). And, as we already mentionned 
in the introduction, since $C$ is a compact subset of the Kasner circle $\mathcal{K}$ which does not contain any of the three special points $T_{1}, T_{2}, T_{3}$, there exists a constant $\nu^{C}>1$ such that, for every $q \in C$ and every $v \in T_{p} \mathcal{K}$, we have $\|D f(p) . v\|_{h} \geq \nu^{C}\|v\|$, where $\|\cdot\|_{h}$ denotes the metric induced on $\mathcal{K}$ by the euclidean metric $h$.

Let $\mathcal{U}:=\Phi^{-1}(\mathcal{V})$. Clearly, $\mathcal{U}$ is a neighborhood of $\widehat{C} \cap M^{C}$ in $M^{C}$. Combining propositions 6.3 and 6.4 , one immediately gets :

Proposition 6.5. The Poincaré map $\Phi^{C}$ is well-defined and $C^{1}$ on $\mathcal{U}$. The compact set $\widehat{C} \cap M^{C}$ is a hyperbolic set for $\Phi$. More precisely, there exists a constant $\nu \in(0,1)$ such that, for every $q \in \widehat{C} \cap M^{C}$,

- $d \Phi^{C}(q) \cdot v=0$ for every $v \in F_{q}^{s}$,

- $d \Phi^{C}(q) \cdot F_{q}^{u}=F_{\Phi^{C}(q)}^{u}$, and $\left\|\left(d \Phi^{C}(q)\right)^{-1} . v\right\|_{g}<\nu\|v\|_{g}$ for every $v \in F_{\Phi^{C}(q)}^{u}$.

Proposition 6.5 shows that the map $\Phi^{C}: M^{C} \rightarrow M^{C}$ and the set $\widehat{C} \cap M^{C}$ satisfy the hypotheses of the stable manifold theorem 6.2 (for any contraction rate $\mu>0$ ). This shows the existence of local stable manifold, with respect to the map $\Phi^{C}$, for the points of $\widehat{C} \cap M^{C}$ :

Theorem 6.6. For every $\varepsilon$ small enough, for every $q \in \widehat{C} \cap M^{C}$, the set

$$
W_{\varepsilon}^{s}\left(\Phi^{C}, q\right):=\left\{r \in M^{C} \mid \text { dist }_{g}\left(\left(\Phi^{C}\right)^{n}(r),\left(\Phi^{C}\right)^{n}(q)\right) \leq \varepsilon \text { for every } n \geq 0\right\}
$$

is a $C^{1}$-embedded disc of dimension 2 in $M^{C}$, tangent to $F_{q}^{s}$ at $q$, depending continuously on $q$ in the $C^{1}$ topology. Moreover, for every constant $\mu>0$, there exists another constant $K$ such that, for every $q \in \widehat{C} \cap M^{C}$, for every $r \in W_{\varepsilon}^{s}\left(\Phi^{C}, q\right)$ and every $n \geq 0$

$$
\operatorname{dist}_{g}\left(\left(\Phi^{C}\right)^{n}(r),\left(\Phi^{C}\right)^{n}(q)\right) \leq K \mu^{n} .
$$

\section{Stable MANifolds For the WAINWRight-HSU VeCtOR FIELD: PROOF OF THEOREM 1.3}

We are left to prove that theorem 6.6 implies our main theorem 1.3.

Consider a point $q \in C$. Then heteroclinic orbit $\mathcal{O}_{q, f(q)}$ intersects the "Poincaré section" $M^{C}$ at one and only one point, that we denote by $\bar{q}$. Note that $\bar{q} \in \widehat{C} \cap M^{C}$. The set $W_{\varepsilon}^{s}\left(\Phi^{C}, \bar{q}\right)$ defined in the statement of theorem 6.6 is a $C^{1}$-embedded two-dimensional disc in the three-dimensional hypersurface with boundary $M^{C}$. This disc is tangent to $F_{\bar{q}}^{s}$ at $\bar{q}$. Since the two-dimensional submanifold $\mathcal{B}_{\mathrm{VII}_{0}} \cup \mathcal{B}_{\mathrm{II}}$ is not tangent to $F_{\bar{q}}^{s}$ at $\bar{q}$, this implies that $W_{\varepsilon}^{s}\left(\Phi^{C}, \bar{q}\right) \cap \mathcal{B}_{\text {IX }}$ contains a $C^{1}$-embedded two-dimensional disc in $M^{C}$. Moreover, this disc depends continuously on $\bar{q}$.

Proposition 7.1. For every point $r$ in $W_{\varepsilon}^{s}\left(\Phi^{C}, \bar{q}\right) \cap \mathcal{B}_{I X}$ :

(1) there is an increasing sequence of times $\left(t_{n}\right)_{n \geq 0}$ such that dist $t_{g}\left(X_{\mathcal{B}}^{t_{n}}(r), f^{n}(q)\right) \underset{n \rightarrow \infty}{\longrightarrow} 0$;

(2) the Hausdorff distance between the piece of orbit $\left\{X_{\mathcal{B}}^{t}(r) ; t_{n} \leq t \leq t_{n+1}\right\}$ and the heteroclinic orbit $\mathcal{O}_{f^{n}(q), f^{n+1}(q)}$ tends to 0 when $n$ goes to $+\infty$. 
Proof. We first prove item 1. According to theorem 6.6, we have

$$
\operatorname{dist}_{g}\left(\left(\Phi^{C}\right)^{n}(r),\left(\Phi^{C}\right)^{n}(\bar{q})\right) \underset{n \rightarrow \infty}{\longrightarrow} 0 .
$$

Together with the continuity of the flow of $X_{\mathcal{B}}$, this shows the existence of a increasing sequence of real numbers $\left(\tau_{n}\right)_{n \geq 0}$ such that

$$
\operatorname{dist}_{g}\left(X_{\mathcal{B}}^{\tau_{n}}\left(\left(\Phi^{C}\right)^{n}(r)\right), \omega\left(\left(\Phi^{C}\right)^{n}(\bar{q})\right)\right) \underset{n \rightarrow \infty}{\longrightarrow} 0 .
$$

Since $r \in \mathcal{B}_{\text {IX }}$, there exists an increasing sequence of times $\left(\alpha_{n}\right)_{n \geq 0}$ such that, for every $n \geq 0$,

$$
\left(\Phi^{C}\right)^{n}(r)=X_{\mathcal{B}}^{\alpha_{n}}(r) .
$$

Since $\bar{q} \in \mathcal{B}_{\mathrm{II}}$, we have, for every $n \geq 0$,

$$
\omega\left(\left(\Phi^{C}\right)^{n}(\bar{q})\right)=f^{n}(\omega(\bar{q}))=f^{n+1}(q) .
$$

For every $n \geq 0$, let $t_{n+1}:=\tau_{n}+\alpha_{n}$. Then $\left(t_{n}\right)_{n \geq 0}$ is an increasing sequence, and

$$
\operatorname{dist}_{g}\left(X_{\mathcal{B}}^{t_{n+1}}(r), f^{n+1}(q)\right) \underset{n \rightarrow \infty}{\longrightarrow} 0 .
$$

This completes the proof of item 1.

To prove item 2, we decompose the piece of orbit $\left\{X_{\mathcal{B}}^{t}(r) ; t_{n} \leq t \leq t_{n+1}\right\}$ into three sub-pieces:

- First, the piece of orbit going from $X_{\mathcal{B}}^{t_{n}}\left(r_{0}\right)$ to $\Delta^{C}\left(\left(\Phi^{C}\right)^{n}(r)\right)$, contained in $V$. Formula (14), together with (33) shows that, for $n$ large, this piece of orbit is close to the heteroclinic orbit $\mathcal{O}_{f^{n}(q), f^{n+1}(q)}$. In particular, for $n$ large, the point $\Delta^{C}\left(\left(\Phi^{C}\right)^{n}(r)\right)$ is close to the heteroclinic orbit $\mathcal{O}_{f^{n}(q), f^{n+1}(q)}$.

- Then, a piece of orbit going from $\Delta^{C}\left(\left(\Phi^{C}\right)^{n}(r)\right)$ to $\left(\Phi^{C}\right)^{n+1}(r)$, contained in $\mathcal{B} \backslash V$. For $n$ large, this piece of orbit is close to the heteroclinic orbit $\mathcal{O}_{f^{n}(q), f^{n+1}(q)}$. Indeed, for $n$ large, the point $\Delta^{C}\left(\left(\Phi^{C}\right)^{n}\left(r_{0}\right)\right)$ is close to the heteroclinic orbit $\mathcal{O}_{f^{n}(q), f^{n+1}(q)}$, and if we write $\left(\Phi^{C}\right)^{n+1}(r)=X_{\mathcal{B}}^{t\left(\Delta^{C}\left(\left(\Phi^{C}\right)^{n}(r)\right)\right)}\left(\Delta^{C}\left(\left(\Phi^{C}\right)^{n}(r)\right)\right)$, then $t\left(\Delta^{C}\left(\left(\Phi^{C}\right)^{n}(r)\right)\right)$ depends continuously on $\Delta^{C}\left(\left(\Phi^{C}\right)^{n}(r)\right)$ (proposition 6.4) and thus is uniformly bounded.

- Finally, a piece of orbit going from $\left(\Phi^{C}\right)^{n+1}(r)$ to $X_{\mathcal{B}}^{t_{n+1}}(r)$, contained in $V$. Formula (14) together with (33) show that, for $n$ large, this piece of orbit is close to the heteroclinic orbit $\mathcal{O}_{f^{n}(q), f^{n+1}(q)}$.

This completes the proof of item 2

\section{Corollary 7.2.}

$$
W^{s}(q)=\bigcup_{t \geq 0} \bigcup_{n \geq 0} X^{-t}\left(W_{\varepsilon}^{s}\left(\Phi^{C},\left(\Phi^{C}\right)^{n}(\bar{q})\right) \cap \mathcal{B}_{I X}\right)
$$

Proof. The inclusion of the set on the right hand side in $W^{s}(q)$ follows from proposition 7.1. The inclusion of $W^{s}(q)$ in the set on the right hand side is an immediate consequence of the definition of $W^{s}(q)$.

We can now complete the proof of our main theorem. 
Proof of theorem 1.3. Fix $\eta>0$, and we set

$$
D_{\mathrm{IX}}^{s}(q):=\bigcup_{-\eta \leq t \leq \eta} X_{\mathcal{B}}^{t}\left(W_{\varepsilon}^{s}\left(\Phi^{C}, \bar{q}\right) \cap \mathcal{B}_{\mathrm{IX}}\right)
$$

According to corollary 7.2, $D_{\mathrm{IX}}^{s}(q)$ is contained in $W^{s}(q)$. Since $\left.W_{\varepsilon}^{s}\left(\Phi^{C}, \bar{q}\right)\right) \cap \mathcal{B}_{\mathrm{IX}}$ is a $C^{1}$-embedded 2-dimensional disc in $M^{C}$, and since the orbits of $X_{\mathcal{B}}$ are transversal to $M^{C}$, we get that $D_{\mathrm{IX}}^{s}(q)$ is a $C^{1}$-embedded 3-dimensional disc in $\mathcal{B}_{\text {IX }}$. Since $\bar{q}$ depends continuously on $q$ (proposition 4.3), and since $W_{\varepsilon}^{s}\left(\Phi^{C}, \bar{q}\right)$ depends continuously on $\bar{q}$ (theorem 6.6), the disc $D_{\mathrm{IX}}^{s}(q)$ depends continuously on $q$.

Remark 7.3. The fact that $W^{s}(q)$ is an $C^{1}$ injectively immersed open disc which depends continuousluy on $q$ (remark 1.4) almost follows from the same arguments. More precisely, theorem 6.6, corollary 7.2 and the transversality of $M^{C}$ to the orbits of $X$ show that $W^{s}(q)$ is an increasing union of $C^{1}$ embedded closed discs which depend continuously on $q$. The only thing which remains to shows is that this increasing union of closed discs is an open disc; this is actually a consequence of the fact that the orbit of $q$ under the Kasner map $f$ is not periodic.

\section{EXISTENCE OF CLOSED FORWARD-INVARIANT APERIODIC SUBSETS OF THE KASNER CIRCLE: PROOF OF PROPOSITION 1.6}

The purpose of this section is to prove proposition 1.6. This proposition should be quite obvious for people with some culture in dynamical systems. Indeed, the Kasner map $f: \mathcal{K} \rightarrow \mathcal{K}$ is a degree -2 map of the circle $\mathcal{K}$. This implies the existence of a continuous degree 1 map $\eta: \mathcal{K} \rightarrow \mathbb{R} / \mathbb{Z}$ such that $\eta \circ f=m_{-2} \circ \phi$ where $m: \mathbb{R} / \mathbb{Z} \rightarrow \mathbb{R} / \mathbb{Z}$ is defined by $m(\theta)=-2 \theta$. Moreover, the Kasner map $f$ is expansive : the norm of the derivative of $f$ (calculated with respect to the metric induced on $\mathcal{K}$ by the rimennian metric $h$ ) is strictly bigger than 1 , except at the three special points $T_{1}, T_{2}, T_{3}$ (where it is equal to 1 ). This implies that the map $\eta: \mathcal{K} \rightarrow \mathbb{R} / \mathbb{Z}$ defined above is one-to-one, that is $f: \mathcal{K} \rightarrow \mathcal{K}$ is topologically conjugated to the map $m: \theta \mapsto-2 \theta$. Finally, it is well-known by experts that, for $|k| \geq 2$, the union of all compact subsets of $\mathbb{R} / \mathbb{Z}$ which are aperiodic for the map $\theta \mapsto k \theta$ is dense in $\mathbb{R} / \mathbb{Z}$. We now give a more detailed proof of the proposition for the readers who may not necessarily be familiar with low-dimensional dynamics.

Proof. Recall that $T_{1}, T_{2}, T_{3}$ are the three Taub points on the Kasner circle $\mathcal{K}$. Let $I_{1}, I_{2}, I_{3}$ be the closures of the three connected components of $\mathcal{K} \backslash\left\{T_{1}, T_{2}, T_{3}\right\}$, the notations being chosen so that $T_{1}$ is not one end of $I_{1}, T_{2}$ is not one end of $I_{2}$, and $T_{3}$ is not an end of $I_{3}$.

We consider the set $\Sigma:=\{1,2,3\}^{\mathbb{N}}$ endowed with the product topology, and the shift map $\sigma: \Sigma \rightarrow \Sigma$ defined by $\sigma\left(a_{0}, a_{1}, a_{2}, \ldots\right)_{i}=\left(a_{1}, a_{2}, a_{3}, \ldots\right)$ (in other words, if $\bar{a}=\left(a_{i}\right)_{i \in \mathbb{N}} \in \Sigma$, then $(\sigma(\bar{a}))_{i}=$ $\left.a_{i+1}\right)$. Let $\Sigma_{0}$ be the subset of $\Sigma$ defined as follows :

$$
\Sigma_{0}=\left\{\bar{a}=\left(a_{i}\right)_{i \in \mathbb{N}} \in \Sigma \text { such that } a_{i+1} \neq a_{i} \text { for every } i\right\} .
$$

Note that $\Sigma_{0}$ is $\sigma$-invariant. We will construct a continuous "almost one-to-one" map $h: \Sigma_{0} \rightarrow \mathcal{K}$ such that $h \circ \sigma=f \circ h$.

Claim. For each sequence $\bar{a}:=\left(a_{i}\right)_{i \in \mathbb{N}}$ in $\Sigma_{0}$, there exists a unique point $p \in \mathcal{K}$ such that $f^{i}(p) \in I_{a_{i}}$ for every $i \geq 0$. 
In order to prove the existence of $p$, one just needs to notice that the image under $f$ of each of the intervals $I_{1}, I_{2}, I_{3}$ is the union of the two other intervals. This implies that the intersection $\bigcap_{i=0}^{N} f^{-i}\left(I_{a_{i}}\right)$ is non-empty for every $N$, and therefore, that the intersection $\bigcap_{i \in \mathbb{N}} f^{-i}\left(I_{a_{i}}\right)$ is non-empty. The existence of $p$ follows. In order to prove the uniqueness of $p$, observe that: for every $\varepsilon>0$, there exists $\nu(\varepsilon)>1$ such that $\left\||D f(p) \||_{h} \geq \nu(\varepsilon)\right.$ for every $x \in \mathcal{K}$ such that $\operatorname{dist}\left(x, T_{i}\right)>\varepsilon$ for $i=1,2,3$. Hence, if $p \neq p^{\prime}$ were two points such that $f^{i}(p) \in I_{a_{i}}$ and $f^{i}\left(p^{\prime}\right) \in I_{a_{i}}$ for every $i \geq 0$, then one would have $\operatorname{dist}\left(f^{i}(p), f^{i}\left(p^{\prime}\right)\right) \rightarrow \infty$; this is absurd since the lengths of $I_{1}, I_{2}$ and $I_{3}$ are finite. Hence there is at most one point $p$ in $\bigcap_{i \in \mathbb{N}} f^{-i}\left(I_{a_{i}}\right)$. This completes the proof of the claim.

Now, we consider the map $h: \Sigma_{0} \rightarrow \mathcal{K}$ which maps a sequence $\bar{a}:=\left(a_{i}\right)_{i \in \mathbb{N}}$ to the unique point $p \in \mathcal{K}$ such that $f^{i}(p) \in I_{a_{i}}$ for every $i \geq 0$. This map $h$ obviously satisfies $h \circ \sigma=f \circ h$. It is continuous (this is an immediate consequence of the continuity of $f$ ) and onto (because the image under $f$ of one of the intervals $I_{1}, I_{2}, I_{3}$ is contained in the union of the two others). It is not one-to-one. For example, the Taub point $T_{3}$ has two pre-images under $h$ : the sequences $(1,2,1,2,1,2, \ldots)$ and $(2,1,2,1,2,1, \ldots)$. More generally, every point $x \in \mathcal{K}$ such that $f^{i_{0}}(p)$ is a Taub point for some integer $i_{0} \geq 0$ (and thus $f^{i}(p)=f^{i_{0}}(p)$ for every $i \geq i_{0}$ ) has two pre-images under $h$, and these two pre-images are preperiodic for $\sigma$. This is the only lack of injectivity of $h:$ if $p \in \mathcal{K}$ has not a single pre-image under $h$, then there exists $i_{0} \geq 0$ such that $f^{i_{0}}(p)$ is a Taub point (this follows from the fact that the intersection between two of the three intervals $I_{1}, I_{2}, I_{3}$ is reduced to a Taub point).

It follows that the image under $h$ of a closed $\sigma$-invariant aperiodic subset of $\Sigma_{0}$ is a closed forwardinvariant aperiodic subset of $\mathcal{K}$. So we are left to prove that the union of all the closed $\sigma$-invariant and aperiodic subsets of $\Sigma_{0}$ is in dense in $\Sigma_{0}$.

A element $\bar{a}=\left(a_{i}\right)_{i \geq 0}$ of $\Sigma_{0}$ is said to be square-free if it does not contain the same word repeated twice : for every $i_{0} \geq 0$ and every $\ell>0$, the word $a_{i_{0}} \ldots a_{i_{0}+\ell-1}$ is different from the word $a_{i_{0}+\ell} \ldots a_{i_{0}+2 \ell-1}$. It is well-known that there exist square-free elements in $\Sigma_{0}$ (such an element may be easily deduced from the well-known Prouhet-Thue-Morse sequence, see for example [1, corollary 1]). Now let $\bar{a}=\left(a_{i}\right)_{i \geq 0} \in \Sigma_{0}$ be square-free, then the $\sigma$-orbit of $\bar{a}$ does not accumulates on any periodic $\sigma$ orbit, hence the closure of the $\sigma$-orbit of $\bar{a}$ is a closed forward-invariant aperiodic subset of $\Sigma_{0}$. Moreover the same is true, if one replaces $\bar{a}$ by a sequence $\bar{a}^{\prime} \in \Sigma_{0}$ which has the same tail as $\bar{a}$ (i.e. there exists $i_{0}$ such that $a_{i}^{\prime}=a_{i}$ for $i \geq i_{0}$ ). The set of all sequences $\bar{a}^{\prime}$ which has the same tail has $\bar{a}$ is obviously dense in $\Sigma_{0}$. Hence the union of all closed $\sigma$-invariant aperiodic subsets of $\Sigma_{0}$ is dense in $\Sigma_{0}$. As explained above, the proposition follows.

\section{REFERENCES}

[1] J.-P. Allouche and J. Shallit. The ubiquitous Prouhet-Thue-Morse sequence. Sequences and their applications, Proceedings of SETA98, C. Ding, T. Helleseth and H. Niederreiter Eds (1999), Springer Verlag, 116.

[2] M. Georgi, J. Härterich, S. Liebscher and K. Webster. Ancient Dynamics in Bianchi Models. Approach to Periodic Cycles. arXiv: 1004.1989.

[3] J. M. Heinzle and C. Uggla. Mixmaster : Fact and Belief.. arXiv: 0901.0776.

[4] J. M. Heinzle and C. Uggla. A new proof of the Bianchi type IX attractor theorem. arXiv:0901.0806.

[5] C. W. Misner. Mixmaster universe. Phys. Rev. Lett. 22 (1969), 1071-1074.

[6] J. Palis and F. Takens. Hyperbolicity and sensitive chaotic dynamics at homoclinic bifurcations. Cambridge University Press, 1993.

[7] A. Rendall. Global dynamics of the mixmaster model. Class. Quantum Grav. 14 (1997), 2341-2356.

[8] H. Ringstrom. Curvature blow up in Bianchi VIII and IX vacuum spacetimes. Class. Quantum. Grav. 17 (2000), $713-731$. 
[9] H. Ringstrom. Bianchi IX attractor. Annales Henri Poincaré 2 (2001), 405-500.

[10] F. Takens. Partially hyperbolic fixed points. Topology 10 (1971), 133-147.

[11] J. Wainwright and G. F. R. Ellis. Dynamical Systems in Cosmology, Cambridge University Press, 1997.

Laboratoire de Mathématiques (UMR 8628), Univ. Paris Sud 11, 91405 Orsay, France.

E-mail address: Francois. Beguin@math.u-psud.fr 


\title{
APERIODIC OSCILLATORY ASYMPTOTIC BEHAVIOR FOR SOME BIANCHI SPACETIMES
}

\author{
FRANÇOIS BÉGUIN
}

\begin{abstract}
We study the asymptotic behavior of vacuum Bianchi type A spacetimes. It has been conjectured that this behavior is driven by a certain circle map, called the Kasner map. As a step towards this conjecture, we prove that some orbits of the Kasner map do indeed attract some solutions of the system of ODEs which describes the behavior of vacuum Bianchi type A spacetimes. The orbits of the Kasner map for which we can prove such a result are those which are not periodic and which do not accumulate on any periodic orbit. This shows the existence of Bianchi spacetimes with a aperiodic oscillatory asymptotic behavior.
\end{abstract}

\section{INTRODUCTION}

A Bianchi spacetime is a cosmological spacetime which is spatially homogeneous. More precisely, a Bianchi spacetime is a spacetime $(M, g)$ such that $M$ is diffeomorphic to the product $G \times I$ of a threedimensional Lie group $G$ and an interval $I$ of $\mathbb{R}$, and $g=h_{t}-d t^{2}$ where $h_{t}$ is a left-invariant riemannian metric on $G \times\{t\} \simeq G$ for every $t \in I$. Hence a Bianchi spacetime can be seen as a one-parameter family $\left(h_{t}\right)_{t \in I}$ of left-invariant riemannian metrics on a given three-dimensional Lie group $G$. A vacuum Bianchi spacetime is a Bianchi spacetime satisfying the vacuum Einstein equation $\operatorname{Ric}_{g}=0$. A Bianchi spacetime is of type $A$ if the corresponding three dimensional Lie group $G$ is unimodular (otherwise it is of type B).

The space of left-invariant riemannian metrics on a given Lie group is finite-dimensional. Therefore, in the restricted context of Bianchi spacetimes, the Einstein equation is simply an ODE on a finite dimensional phase space. A classical and convenient way to write this ODE is to use the so-called WainwrightHsu variables $\left(\Sigma_{1}, \Sigma_{2}, \Sigma_{3}, N_{1}, N_{2}, N_{3}\right)$. The corresponding phase space is the four-dimensional manifold

$$
\mathcal{B}=\left\{\left(\Sigma_{1}, \Sigma_{2}, \Sigma_{3}, N_{1}, N_{2}, N_{3}\right) \in \mathbb{R}^{6} \mid \Sigma_{1}+\Sigma_{2}+\Sigma_{3}=0, \Omega=0\right\}
$$

where

$$
\Omega=6-\left(\Sigma_{1}^{2}+\Sigma_{2}^{2}+\Sigma_{3}^{2}\right)+\frac{1}{2}\left(N_{1}^{2}+N_{2}^{2}+N_{3}^{2}\right)-\left(N_{1} N_{2}+N_{1} N_{3}+N_{2} N_{3}\right) .
$$

Work supported by ANR project GEODYCOS. 
and the vacuum Einstein equation reads

$$
\text { (S) }\left\{\begin{aligned}
\Sigma_{1}^{\prime} & =(2-q) \Sigma_{1}-R_{1} \\
\Sigma_{2}^{\prime} & =(2-q) \Sigma_{2}-R_{2} \\
\Sigma_{3}^{\prime} & =(2-q) \Sigma_{3}-R_{3} \\
N_{1}^{\prime} & =-\left(q+2 \Sigma_{1}\right) N_{1} \\
N_{2}^{\prime} & =-\left(q+2 \Sigma_{2}\right) N_{2} \\
N_{3}^{\prime} & =-\left(q+2 \Sigma_{3}\right) N_{3}
\end{aligned}\right.
$$

where

$$
\begin{aligned}
q & =\frac{1}{3}\left(\Sigma_{1}^{2}+\Sigma_{2}^{2}+\Sigma_{3}^{2}\right) \\
R_{1} & =\frac{1}{3}\left(2 N_{1}^{2}-N_{2}^{2}-N_{3}^{3}+2 N_{2} N_{3}-N_{1} N_{3}-N_{1} N_{2}\right) \\
R_{2} & =\frac{1}{3}\left(2 N_{2}^{2}-N_{3}^{2}-N_{1}^{3}+2 N_{3} N_{1}-N_{2} N_{1}-N_{2} N_{3}\right) \\
R_{3} & =\frac{1}{3}\left(2 N_{3}^{2}-N_{1}^{2}-N_{2}^{3}+2 N_{1} N_{2}-N_{3} N_{2}-N_{3} N_{1}\right)
\end{aligned}
$$

(see, for example, [3] for the construction of the Wainwright-Hsu variables and the derivation of the above equations). In other words, vacuum Bianchi type A spacetimes can be seen as the parametrized curves in $\mathcal{B}$ that are solutions of the above system of ODEs. We will call Wainwright-Hsu vector field, and denote by $X$, the vector field on $\mathcal{B}$ corresponding to this system of ODE. We will denote by $X^{t}$ the time $t$ map of the flow of $X$. We will denote $\mathcal{B}^{+}$the subset of $\mathcal{B}$ made of the points for which $N_{1}, N_{2}$ and $N_{3}$ are non-negative. To measure the distances on $\mathcal{B}$, we will use the riemannian metric $h=d \Sigma_{1}^{2}+d \Sigma_{2}^{2}+d \Sigma_{3}^{2}+d N_{1}^{2}+d N_{2}^{2}+d N_{3}^{2}$ on $\mathcal{B}$.

Remark 1.1. We have chosen the "anti-physical" time orientation: Bianchi spacetimes will be future incomplete, but not necessarily past incomplete. With this "anti-physical" convention, the so-called mixmaster attractor (see below) is indeed an attractor (and not a repellor).

The phase space $\mathcal{B}$ admits a natural stratification, according to the signs of the variable $N_{1}, N_{2}, N_{3}$, this stratification being invariant under the flow of $X$. The different strata correspond to the different types of three-dimensional Lie groups. The behavior of the orbits of $X$ contained in the one, two and three-dimensional strata (so-called, type $\mathrm{I}, \mathrm{II}_{,} \mathrm{VI}_{0}$ and $\mathrm{VII}_{0}$ orbits) is fully-understood. On the contrary, there is almost no rigorous result on the behavior of the orbits in the four dimensional biggest strata (co-called type VIII and IX orbits).

The smallest stratum is called the Kasner circle, denoted by $\mathcal{B}_{1}$ or $\mathcal{K}$, and corresponds to the case where the Lie group $G$ is $\mathbb{R}^{3}$. It is made of the points of $\mathcal{B}$ for which $N_{1}=N_{2}=N_{3}=0$ :

$$
\begin{aligned}
\mathcal{K} & =\left\{\left(\Sigma_{1}, \Sigma_{2}, \Sigma_{3}, N_{1}, N_{2}, N_{3}\right) \in \mathcal{B} ; N_{1}=N_{2}=N_{3}=0\right\} \\
& =\left\{\left(\Sigma_{1}, \Sigma_{2}, \Sigma_{3}, N_{1}, N_{2}, N_{3}\right) \in \mathbb{R}^{6} ; N_{1}=N_{2}=N_{3}=0 \quad \Sigma_{1}+\Sigma_{2}+\Sigma_{3}=0 \quad q=2\right\}
\end{aligned}
$$


This is a euclidean circle in $\mathbb{R}^{6}$. The orbits of $X$ contained in $\mathcal{B}_{\mathrm{I}}=\mathcal{K}$ are called type I orbits. The vector field $X$ vanishes on $\mathcal{K}$. Hence, every type I orbit is a fixed point. There are three points on the Kasner circle, called the special points or the Taub points which play a very important role in every attempt to understand the behavior of the solution of the Wainwright-Hsu equations; these are the points for which $\left(\Sigma_{1}, \Sigma_{2}, \Sigma_{3}\right)$ is equal to $(2,-1,-1)$ or $(-1,2,-1)$ or $(-1,-1,2)$; there are usually denoted by $T_{1}, T_{2}, T_{3}$. For every point $p \in \mathcal{K}$, the derivative $D X(p)$ has four distinct eigendirections: the direction tangent to the Kasner circle which corresponds to a zero eigenvalue since $X$ vanishes on $\mathcal{K}$, and the three directions $\frac{\partial}{\partial N_{1}}, \frac{\partial}{\partial N_{2}}$ and $\frac{\partial}{\partial N_{3}}$ which are respectively associated to the eigenvalues $-\left(2+2 \Sigma_{1}\right),-(2+$ $\left.2 \Sigma_{2}\right)$ and $-\left(2+2 \Sigma_{3}\right)$. If $p$ is one of the three special points $T_{1}, T_{2}, T_{3}$, then two of the three eigenvalues $-\left(2+2 \Sigma_{1}\right),-\left(2+2 \Sigma_{2}\right),-\left(2+2 \Sigma_{3}\right)$; hence, the derivative of $D X(p)$ has a triple zero eigenvalue. If $p$ is not one of the three special points $T_{1}, T_{2}, T_{3}$, then the eigenvalues $-\left(2+2 \Sigma_{1}\right),-\left(2+2 \Sigma_{2}\right),-\left(2+2 \Sigma_{3}\right)$ are pairwise distinct, two of them are negative, and the other one is positive; in other words, the derivative $D X(p)$ has two distinct negative eigenvalue, a multiplicity one eigenvalue, and a positive eigenvalue.

The next stratum is denoted by $\mathcal{B}_{\mathrm{II}}$ and correspond to the case where $G$ is the Heisenberg group. It is made of the points of $\mathcal{B}$ for which exactly two of the $N_{i}$ 's are equal to zero:

$$
\mathcal{B}_{1} \cup \mathcal{B}_{\mathrm{II}}=\left\{\left(\Sigma_{1}, \Sigma_{2}, \Sigma_{3}, N_{1}, N_{2}, N_{3}\right) \in \mathcal{B} ; N_{1}=N_{2}=0 \text { or } N_{1}=N_{3}=0 \text { or } N_{2}=N_{3}=0\right\}
$$

One can easily check that $\mathcal{B}_{\mathrm{I}} \cup \mathcal{B}_{\mathrm{II}}$ is the union of three ellipsoids which intersect along the Kasner circle $\mathcal{K}=\mathcal{B}_{\mathrm{I}}$. The vector field $X$ commutes with the transformation $\left(N_{1}, N_{2}, N_{3}\right) \mapsto\left(-N_{1},-N_{2},-N_{3}\right)$, so we can work in $\mathcal{B}_{\mathrm{II}} \cap \mathcal{B}^{+}$to simplify the discussion. The intersection $\mathcal{B}_{\mathrm{II}} \cap \mathcal{B}^{+}$is the union of three disjoint hemi-ellipsoids bounded by the Kasner circle. The orbits in $\mathcal{B}_{2}$ are called type II orbits. Type II orbits can be calculated explicitly (see for example [3]). It appears that every type II is a heteroclinic orbit connecting a point of $\mathcal{K} \backslash\left\{T_{1}, T_{2}, T_{3}\right\}$ to another point of $\mathcal{K} \backslash\left\{T_{1}, T_{2}, T_{3}\right\}$. Moreover, for point $p \in \mathcal{K} \backslash\left\{T_{1}, T_{2}, T_{3}\right\}$, there is exactly one type II orbit in $\mathcal{B}^{+}$which "starts" at $p$ (this orbit is tangent to the eigendirection associated to the positive eigenvalue of $D X(p)$ ) and two type II orbits in $\mathcal{B}^{+}$which "starts" at $p$ (these orbits are tangent to the eigendirections associated to the negative eigenvalues of $D X(p)$ ). The Kasner map $f: \mathcal{K} \rightarrow \mathcal{K}$ is defined as follows. The three special points $T_{1}, T_{2}, T_{3}$ are fixed under $f$. For every point $p \in \mathcal{K} \backslash\left\{T_{1}, T_{2}, T_{3}\right\}$, one considers the unique type II orbit which "starts" at $p$; this heteroclinic orbit connects $p$ to another point $q \in \mathcal{K} \backslash\left\{T_{1}, T_{2}, T_{3}\right\}$, which is by definition the point $f(p)$. We will denote by $\mathcal{O}_{p, f(p)}$ the unique type II heteroclinic orbit connecting $p$ to $f(p)$ in $\mathcal{B}^{+}$. The Kasner map $f$ admit a nice geometric description. Consider the equilateral triangle which is tangent to $\mathcal{K}$ at the three special points $T_{1}, T_{2}, T_{3}$. Denote by $M_{1}, M_{2}, M_{3}$ the vertices of this triangle. For $p \in \mathcal{K} \backslash\left\{T_{1}, T_{2}, T_{3}\right\}$, let $M_{i}$ be the vertex of $\mathcal{K}$ which is the closest to $p$. The line $\left(M_{i} p\right)$ and intersects the circle $\mathcal{K}$ at two points: the point $p$ and the point $f(p)$. See figure 1. Using this geometric description, it is easy to see $f$ is $C^{1}$. Let us consider the derivative of $f$ with the respect to the arc length on $\mathcal{K}$ defined by the riemannian metric $h$. Then the norm of the derivative of the Kasner map at the three special points is equal to 1 . But, for every $p \in \mathcal{K} \backslash\left\{T_{1}, T_{2}, T_{3}\right\}$ the modulus of the derivative $f$ at $p$ is strictly bigger than 1 . The Kasner map $f$ is non-uniformly expanding : for every compact set $C$ in $\mathcal{K} \backslash\left\{T_{1}, T_{2}, T_{3}\right\}$ there exists a constant $\nu_{C}>1$ such that $\left\||D f(p) \||_{h} \geq \nu_{C}\right.$ for every $p$ in $C$.

The strata $\mathcal{B}_{\mathrm{VI}_{0}}$ and $\mathcal{B}_{\mathrm{VII}_{0}}$ are three-dimensional; $\mathcal{B}_{\mathrm{VI}_{0}}$ is made of the points of $\mathcal{B}$ for which exactly one of the $N_{i}$ 's is equal to zero, the two others being of different signs; $\mathcal{B}_{\mathrm{VII}_{0}}$ is made of the points of $\mathcal{B}$ for which exactly one of the $N_{i}$ 's is equal to zero, the two others being of the same sign. They correspond respectively to the case where $G=\mathrm{O}(1,1) \ltimes \mathbb{R}^{2}$ and $G=O(2) \ltimes \mathbb{R}^{2}$. Observe that $\mathcal{B}_{\mathrm{VI}_{0}}$ does not intersect $\mathcal{B}^{+}$. 


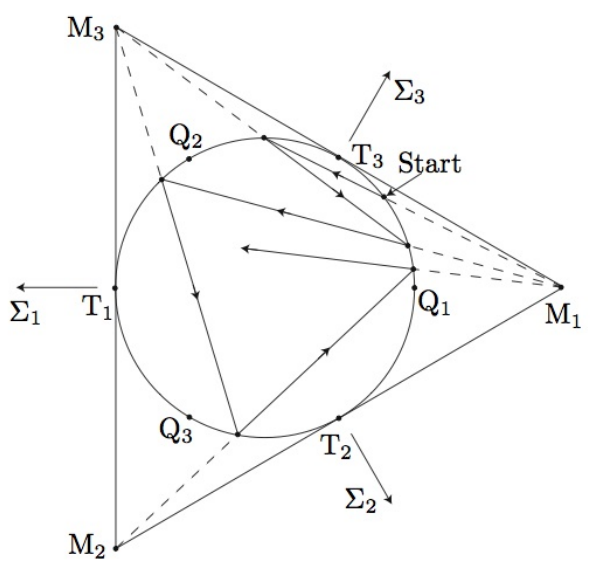

FIGURE 1. Construction of an orbit of the Kasner map

Finally the strata $\mathcal{B}_{\text {VIII }}$ and $\mathcal{B}_{\text {IX }}$ are four-dimensional (these are open subsets of $\mathcal{B}$ ). They correspond to the points of $\mathcal{B}$ for which none of the $N_{i}$ 's is equal to zero. In $\mathcal{B}_{\text {VIII }}$ the $N_{i}$ 's do not have the same sign, whereas in $\mathcal{B}_{\text {IX }}$ the three $N_{i}$ 's have the same sign. These strata correspond respectively to the cases where $G=\operatorname{SL}(2, \mathbb{R})$ and $G=\mathrm{SO}(3, \mathbb{R})$. Observe that $\mathcal{B}_{\text {VIII }}$ does not intersect $\mathcal{B}^{+}$

Misner has conjectured that the dynamics of type IX orbits "is driven" by the Kasner map ([5]). The idea is that each "heteroclinic chain" $q \cup \mathcal{O}_{q, f(q)} \cup f(q) \cup \mathcal{O}_{f(q), f^{2}(q)} \cup \ldots$ should attract some Bianchi IX, and that "generic" Bianchi IX orbits should correspond to "generic" orbits of the Kasner map (see [3] for a detailed discussion various possible precise meanings of this conjecture) (recall that by $\mathcal{O}_{f^{n}(q), f^{n+1}(q)}$ is the type II heteroclinic orbit connecting $f^{n}(q)$ to $f^{n+1}(q)$ in $\mathcal{B}^{+}$.

In 2001, Ringström proved that $\mathcal{A}:=\mathcal{K} \cup \mathcal{B}_{\mathrm{II}}$ is a global attractor : for "almost every" point $q$ in $\mathcal{B}_{\text {IX }}$, the distance from the orbit of $q$ to $\mathcal{A}$ tends to 0 as the time goes to $\infty$ ([9], see also [4]). Although remarkable, Ringström's theorem does not tell anything on the relation between the dynamics of Bianchi orbits and the dynamics the Kasner map. The purpose of the present paper is to prove that "there are many point $q \in \mathcal{K}$ such that the "heteroclinic chain $q \cup \mathcal{O} q, f(q) \cup f(q) \cup \mathcal{O}_{f(q), f^{2}(q)} \cup \ldots$ does attract some Bianchi IX orbits”.

Definition 1.2. For $q \in \mathcal{K}$, we call stable manifold of $q$, and we denote by $W^{s}(q)$, the set of all points $r \in \mathcal{B}$ for which there exists an increasing sequence of real numbers $\left(t_{n}\right)_{n \geq 0}$ such that

$$
\operatorname{dist}\left(X^{t_{n}}(r), f^{n}(q)\right) \underset{n \rightarrow \infty}{\rightarrow} 0
$$

and such that the Hausdorff distance between the piece of orbit $\left\{X^{t}(r) ; t_{n} \leq t \leq t_{n+1}\right\}$ and the type II heteroclinic orbit $\mathcal{O}_{f^{n}(q), f^{n+1}(q)}$ tends to 0 as $n \rightarrow \infty$.

A subset $C$ of $\mathcal{K}$ is said to be $f$-invariant if $f(C) \subset C$. It is said to be aperiodic if it does not contain any periodic orbit for $f$. Our main result can be stated as follows:

Theorem 1.3. If $q \in \mathcal{K}$ is contained in a closed $f$-invariant aperiodic subset of $\mathcal{K}$, then the stable manifold $W^{s}(q)$ contains a $C^{1}$ embedded three-dimensional disc $D_{I X}^{s}(q)$ embedded in $\mathcal{B}_{I X}$. This disc can be chosen to depend continuously on $q$ when $q$ ranges in a closed $f$-invariant aperiodic subset of $\mathcal{K}$. 
Remark 1.4. The analog of theorem 1.3 for $\mathcal{B}_{\text {VIII }}$ instead of $\mathcal{B}_{\text {IX }}$ also holds. We will focus on $\mathcal{B}_{\text {IX }}$ to simplify the notations.

Observe that the hypothesis of theorem 1.3 is satisfied by a dense set of points in $\mathcal{K}$ :

Proposition 1.5. The union of all the closed $f$-invariant aperiodic subsets of $\mathcal{K}$ is in dense in $\mathcal{K}$.

Remark 1.6. Let $E$ be the union of all the closed, forward invariant, aperiodic subsets of $\mathcal{K}$. According to the above proposition, $E$ is dense in $\mathcal{K}$. Observe nevertheless that $E$ is a "small" subset of $\mathcal{K}$ both from the topological viewpoint (it is a meager set) and from the measurable viewpoint (it has zero Lebesgue measure). Also observe that theorem 1.3 does not tell that $W^{s}(q)$ depends continuously on $q$ when $q$ ranges in $E$. So, we do not know whether the union of all the stable manifolds $W^{s}(q)$, where $q$ ranges $E$, is dense in $\mathcal{B}$ (or in an open subset of $\mathcal{B}$ ) or not.

The origin of the hypothesis of theorem 1.3 is purely technical: if $p$ belongs to a $f$-invariant aperiodic compact subset of $\mathcal{K}$, we can find a coordinate system in which the vector field $X$ is "linear"; this makes the estimates on the flow of $X$ near such a point much easier. We do not know if such linearizing coordinate systems exist near a point of $\mathcal{K}$ which is periodic or preperiodic under the Kasner map. Nevertheless, it should be noticed that the estimates on the flow of $X$ that we need to construct stable manifolds are much weaker than the existence of a linearizing coordinate system. So, we do think that theorem 1.3 can be extended to any point $q \in \mathcal{K}$ such that the orbit of $q$ under the Kasner map does not accumulate one any of the three special points $T_{1}, T_{2}, T_{3}$. Understanding the behaviour of the orbits which pass arbitrary close to the three special points seems to be a much harder problem.

As we were finishing to write the present paper, M. Georgi, J. Härterich, S. Liebscher and K. Webster put on arXiv a preprint in which they prove that the period three orbits of $f$ admit non-trivial stable manifolds ([2]). At the end of this preprint, they claim that their techniques can be used to extend their result to any orbit of $f$ which does not accumulate one any of the three special points $T_{1}, T_{2}, T_{3}$. Such a extension would imply our result.

Let us try to give a rough idea of our proof of theorem 1.3. Consider a point $p$ on the Kasner circle $\mathcal{K}$ which is not preperiodic under the Kasner map $f$. Using the link between the behavior of the orbit of $p$ under $f$, the continued fraction development of the Kasner parameter $u(p)$, and the expression of the eigenvalues of $D X(p)$ in terms of the Kasner parameter $u(p)$, we show that the eigenvalues of $D X(p)$ are independent over $\mathbb{Q}$. Then, using a generalization of Sternberg's theorem due to Takens, we obtain a $C^{1}$ coordinate system on a neighborhood $V_{p}$ of $p$ such that $X$ is "essentially linear" in this coordinate system. Since $X$ is "essentially linear", we can compute explicitly the flow of $X$. This leads to the following important observation: when an orbit of $X$ passes in the neighborhood $V_{p}$, the distance of this orbit to the mixmaster attractor decreases super-linearly, while the drift in the direction tangent to the attractor is as small as one wishes. Now consider a closed $f$-invariant aperiodic subset $C$ of $\mathcal{K}$. One can find a finite number of points $p_{1}, \ldots, p_{n}$ in $C$ such that the neighborhoods $V_{p_{1}}, \ldots, V_{p_{n}} \operatorname{cover} C$. When a type IX orbit goes through one of the neighborhoods $V_{p_{1}}, \ldots, V_{p_{n}}$ the distance of this orbit to the mixmaster attractor decreases super-linearly. Of course, this orbit will also do some excursions outside $V_{p_{1}} \cup \cdots \cup V_{p_{n}}$, but the duration of these excursion is uniformly bounded (at least when the orbit is close to a type II heteroclinic orbit connecting a point of $C$ to another point of $C$ ). So the effect of these excursions outside $V_{p_{1}}, \ldots, V_{p_{n}}$ can be neglected when compared to the super-linear contraction 
that occurs inside $V_{p_{1}}, \ldots, V_{p_{n}}$. All in all, this will allow us to show that the drift of the orbits in the direction tangential to the mixmaster attractor $\mathcal{A}$ can be neglected when compared to the contraction of the distance to this attractor. This will lead the existence of stable manifolds.

Sections $2 \ldots 7$ are devoted to the proof of theorem 1.3 . Proposition 1.5 will be proved in section 8 .

Acknowledgements. I would like to thank Lars Andersson for some stimulating discussion, and for his encouragements to write the present paper.

\section{TAKENS LINEARIZATION THEOREM}

Let $Y$ be a $C^{\infty}$ vector field on a manifold $\mathcal{M}$, and $p$ be a point of $\mathcal{M}$ such that $Y(p)=0$. The linear space $T_{p} \mathcal{M}$ admits a unique decomposition

$$
T_{p} \mathcal{M}=E_{p}^{s} \oplus E_{p}^{c} \oplus E_{p}^{u}
$$

where $E_{p}^{s}, E_{p}^{c}, E_{p}^{u}$ are $D Y(p)$-invariant linear subspaces, the real parts of the eigenvalues of the restriction of $D Y(p)$ to $E_{p}^{s}$ (resp. $E_{p}^{c}, E_{p}^{u}$ ) are negative (resp. equal to 0, positive). We shall denote by $s, c$ and $u$ the dimensions of the linear subspaces $E_{p}^{s}, E_{p}^{c}$ and $E_{p}^{u}$.

Definition 2.1. The vector field $Y$ satisfies Sternberg's condition at $p$ if the eigenvalues of $D Y(p)_{\mid E_{p}^{s} \oplus E_{p}^{u}}$ (counted with multiplicities) are independent over $\mathbb{Q}$.

F. Takens as proved the following generalization of the classical Sternberg's linearization theorem :

Theorem 2.2 (see [10], page 144). Assume that $Y$ satisfies Sternberg's condition at $p$. Then, for every $r \geq 0$, one can find a neighborhood $U$ and a $C^{r}$ coordinate system $\left(x_{1}, \ldots, x_{s}, y_{1}, \ldots, y_{c}, z_{1}, \ldots, z_{u}\right)$ on $U$ centered at $p$, such that, in this coordinate system, $Y$ reads:

$$
Y=\sum_{i, j=1}^{s} a_{i, j}\left(y_{1}, \ldots, y_{c}\right) x_{j} \frac{\partial}{\partial x_{i}}+\sum_{i=1}^{c} \phi_{i}\left(y_{1}, \ldots, y_{c}\right) \frac{\partial}{\partial y_{i}}+\sum_{i, j=1}^{u} b_{i, j}\left(y_{1}, \ldots, y_{c}\right) z_{j} \frac{\partial}{\partial z_{i}}
$$

where the eigenvalues of the matrix $\left(a_{i, j}(0, \ldots, 0)\right)_{1 \leq i, j \leq s}$ have negative real parts, the eigenvalues of the matrix $\left(\partial \phi_{i} / \partial y_{j}(0, \ldots, 0)\right)_{1 \leq i, j \leq c}$ are on the imaginary axis, and the eigenvalues of $\left(b_{i, j}(0, \ldots, 0)\right)_{1 \leq i, j \leq u}$ have positive real parts.

Remarks 2.3. In general, the size of the neighborhood $U$ does depend on the integer $r$ in general, and it is not possible to find any $C^{\infty}$ local coordinate system centered at $p$ such that (1) holds.

The name "Takens linearization theorem" is slightly incorrect: the vector field $Y$ is not linear in the $\left(x_{1}, \ldots, x_{s}, y_{1}, \ldots, y_{c}, z_{1}, \ldots, z_{u}\right)$ coordinate system. Nevertheless, observe that the submanifold defined by $\left(y_{1}=\cdots=y_{c}=0\right)$ is invariant under the flow of $Y$, and the restriction of $Y$ to this submanifold is linear. For $\left(y_{1}^{0}, \ldots, y_{c}^{0}\right) \neq(0, \ldots, 0)$, the submanifold defined by $\left(y_{1}, \ldots, y_{c}\right)=\left(y_{1}^{0}, \ldots, y_{c}^{0}\right)$ is not invariant under the flow of $Y$ in general, but the projection of $Y$ on the tangent space of this submanifold is linear.

The vectors $\frac{\partial}{\partial x_{1}}(p), \ldots, \frac{\partial}{\partial x_{s}}(p)$ span the subspace $E_{p}^{s}$, the vectors $\frac{\partial}{\partial y_{1}}(p), \ldots, \frac{\partial}{\partial y_{c}}(p)$ span the subspace $E_{p}^{c}$ and the vectors $\frac{\partial}{\partial z_{1}}(p), \ldots, \frac{\partial}{\partial z_{u}}(p)$ span the subspace $E_{p}^{u}$ : this follows from equality (1) together with the signs of the real parts of the eigenvalues of the matrices $\left(a_{i, j}(0, \ldots, 0)\right)_{1 \leq i, j \leq s}$, $\left(\partial \phi_{i} / \partial y_{j}(0, \ldots, 0)\right)_{1 \leq i, j \leq c}$ and $\left(b_{i, j}(0, \ldots, 0)\right)_{1 \leq i, j \leq u}$. 


\section{LINEARIZATION OF THE VECTOR FIELD $X$ NEAR A POINT OF THE KASNER CIRCLE WHICH IS NOT PREPERIODIC}

Recall that $X$ denotes the vector field on $\mathcal{B}$ whose orbits are the solution of the system of ODEs (S). We will prove that the vector field $X$ satisfies Sternberg's condition at every point of the Kasner circle which is not preperiodic under the Kasner map $f$. For this purpose, we need to introduce the so-called Kasner parameter. If $q=\left(\Sigma_{1}(q), \Sigma_{2}(q), \Sigma_{3}(q), 0,0,0\right)$ is a point of the Kasner circle, the Kasner parameter $u(q) \in[1, \infty]$ of $q$ is defined by

$$
2-\Sigma_{1}(q) \Sigma_{2}(q) \Sigma_{3}(q)=27 \frac{u(q)^{2}(1+u(q))^{2}}{\left(1+u(q)+u(q)^{2}\right)^{3}} .
$$

The map $q \mapsto u(q)$ is not one-to-one: indeed the left hand side of the above equality is invariant under permutation of the $\Sigma_{1}(q), \Sigma_{2}(q), \Sigma_{3}(q)$. Nevertheless, $\Sigma_{1}(q), \Sigma_{2}(q), \Sigma_{3}(q)$ are characterized up to permutation by $u(q)$. Hence, the eigenvalues $-\left(2+2 \Sigma_{1}(q)\right),-\left(2+2 \Sigma_{2}(q)\right),-\left(2+2 \Sigma_{3}(q)\right)$ of $D X(q)$ are characterized up to permutation by the Kasner parameter $u(q)$. More precisely:

$$
\begin{aligned}
& \left\{-\left(2+2 \Sigma_{1}(q)\right),-\left(2+2 \Sigma_{2}(q)\right),-\left(2+2 \Sigma_{3}(q)\right)\right\} \\
= & \left\{\frac{u(q)}{1+u(q)+u^{2}(q)},-\frac{1+u(q)}{1+u(q)+u^{2}(q)},-\frac{u(q)(1+u(q))}{1+u(q)+u^{2}(q)}\right\}
\end{aligned}
$$

(this can be deduced from (2), together with the equalities $\Sigma_{1}(q)+\Sigma_{2}(q)+\Sigma_{3}(q)=0$ and $\Sigma_{1}^{2}(q)+$ $\left.\Sigma_{2}^{2}(q)+\Sigma_{3}^{2}(q)=6\right)$. Note that $u(q)=\infty$ if and only if $q$ is one of the three special points $T_{1}, T_{2}, T_{3}$. The main advantage of the Kasner parameter is the fact that the Kasner map $f$ admits a nice expression in terms of this parameter. For every $q \in \mathcal{K}$, one has $u(f(q))=\bar{f}(u(q))$ where $\bar{f}:[1,+\infty] \rightarrow[1, \infty]$ is defined by

$$
\bar{f}(u)= \begin{cases}\infty & \text { if } u=1 \text { or } \infty \\ u-1 & \text { if } u(q) \geq 2 \\ \frac{1}{u-1} & \text { if } 1<u(q) \leq 2\end{cases}
$$

From now on until the end of the section, we consider a point $p \in \mathcal{K} \backslash\left\{T_{1}, T_{2}, T_{3}\right\}$. As we already pointed out in the introduction, the derivative $D X(p): T_{p} \mathcal{B} \rightarrow T_{p} \mathcal{B}$ has a four distinct (multiplicity one) real eigenvalues: two negative eigenvalues (corresponding to the directions of the two type II in $\mathcal{B}^{+}$which "arrive" at $p$ ), a multiplicity one zero eigenvalue corresponding to the direction of the Kasner circle, and one positive eigenvalue corresponding to the unique type II orbit in $\mathcal{B}^{+}$which "starts" at $p$. We will denote the non-zero eigenvalues of $p$ by $\lambda_{s}(p), \lambda_{s}^{\prime}(p), \lambda_{u}(p)$ with $\lambda_{s}(p)<\lambda_{s}^{\prime}(p)<0<\lambda_{u}(p)$. Recall that $\left(\lambda_{s}(p), \lambda_{s}^{\prime}(p), \lambda_{u}(p)\right)=\left(\left(2+2 \Sigma_{1}(q)\right),-\left(2+2 \Sigma_{2}(q)\right),-\left(2+2 \Sigma_{3}(q)\right)\right)$ up to permutation). From (2), we easily deduce:

$$
\left(\lambda_{s}(p), \lambda_{s}^{\prime}(p), \lambda_{u}(p)\right)=\left(-\frac{u(p)(1+u(p))}{1+u(p)+u^{2}(p)},-\frac{1+u(p)}{1+u(p)+u^{2}(p)}, \frac{u(p)}{1+u(p)+u^{2}(p)}\right)
$$

The following observation will play a key role in the proof of our main theorem 1.3: 
Proposition 3.1. The contraction rate of $D X(p)_{\mid E_{p}^{s}}$ dominates the dilatation rate of $D X(p)_{\mid E_{p}^{u} \text {, that is }}$

$$
\left|\frac{\lambda_{s}(p)}{\lambda_{u}(p)}\right|>1 \quad \text { and } \quad\left|\frac{\lambda_{s}^{\prime}(p)}{\lambda_{u}(p)}\right|>1
$$

Proof. This is an immediate consequence of (5) and of the fact that $\frac{1+u}{u}>1$ and $\frac{u(1+u)}{u}=1+u>1$ for every $u \geq 1$.

The statement below gives some necessary and sufficient condition for the vector field $X$ to satisfy Sternberg's condition at $p$, in terms of the behavior of the orbit of $p$ under the Kasner map. The hypothesis of our main theorem 1.3 comes directly from this condition.

Proposition 3.2. The three following conditions are equivalent :

(1) the vector field $X$ satisfies Sternberg's condition at $p$;

(2) the Kasner parameter $u(p)$ is neither a rational, nor a quadratic irrational number;

(3) $p$ is not a preperiodic point for the Kasner map $f$.

Proof. Let us prove $1 \Leftrightarrow 2$. The vector field $X$ satisfies Sternberg's condition at $p$ if and only if the eigenvalues $\lambda_{s}(p), \lambda_{s}^{\prime}(p), \lambda_{u}(p)$ are independent over $\mathbb{Q}$. Using formulae (5), one sees that this is equivalent to the fact that the numbers $u(p), 1+u(p)$ and $u(p)(1+u(p))$ are independent over $\mathbb{Q}$. Clearly, this is equivalent to the fact $u(p)$ is neither a rational number nor a quadratic irrational number.

Now, let us prove $3 \Leftrightarrow 2$. For this purpose, we will use the action of Kasner map $f$ on the Kasner map. Recall that, for every $q$ on the Kasner circle, one has $u(f(q))=\bar{f}(u(q))$ where $\bar{f}:[1,+\infty] \rightarrow[1, \infty]$ is given by (4). Every orbit of $\bar{f}$ which does not end on $\infty$, passes an infinite number of times in the interval $[1,2]$. Let $k:(1,2] \rightarrow(1,2]$ be the return time function of $\bar{f}$ in the interval $(1,2]$, and $\bar{F}:(1,2] \rightarrow(1,2]$ be the first return map of $\bar{f}$ in the interval $(1,2]$, that is

$$
k(u)=\inf \left\{n>0 \text { such that } \bar{f}^{n}(u) \in(1,2]\right\} \quad \text { and } \quad \bar{F}(u)=\bar{f}^{k(u)}(u) .
$$

Then,

$$
k(u)=\left\lfloor\frac{1}{u-1}\right\rfloor-1 \text { and } \bar{F}(u)=\left\{\frac{1}{u-1}\right\}+1,
$$

where $\lfloor x\rfloor$ is the integer part of $x$ and $\{x\}:=x-\lfloor x\rfloor$ is the fractional part of $x$. Observe that a point $p \in \mathcal{K}$ is preperiodic for the Kasner map $f$ if and only if $u(p)$ is a preperiodic point for $\bar{F}$. Observe that $\bar{F}$ is just the Gauss map $u \mapsto\left\{\frac{1}{u}\right\}$ conjugated by the translation $u \mapsto u+1$, and that $k(u-1)+1$ is the first term of the continuous development fraction of $u$. On the one hand, it is well-known (and very easy to prove) the preperiodic points of the Gauss map $u \mapsto\left\{\frac{1}{u}\right\}$ are exactly the real numbers $u \in(0,1]$ such that the sequence of integers which appear in the continuous fraction development of $u$ is finite or preperiodic. It follows that a point $p \in \mathcal{K}$ is preperiodic for the Kasner map $f$ if and only if the continuous fraction development of $u(p)$ is finite or preperiodic. On the other hand, it is well-known that the continuous fraction development of $u \in \mathbb{R}$ is finite if and only if $u$ is a rational number, and that the continuous fraction development of $u$ is preperiodic if and only if $u$ is a quadratic irrational number. This shows that $p \in \mathcal{K}$ is a preperiodic for the Kasner map $f$ if and only if $u(p)$ is a rational or a quadratic irrational number. 
According to proposition 3.2, if $p$ is not a preperiodic point for the Kasner map, the hypotheses of Takens linearization theorem are satisfied by the vector field $X$ at $p$. This theorem provides us with local coordinates near $p$ in which the vector field $X$ has a very simple expression :

Proposition 3.3. Assume that the point $p$ is not eventually periodic under the Kasner map. Then there exists a neighborhood $U$ of $p$ in $\mathcal{B}$ and a $C^{1}$ coordinate system $\left(x, x^{\prime}, y, z\right)$ on $U$ centered $p$, centered at $p$, and such that, in this coordinate system, $X$ reads :

$$
X\left(x, x^{\prime}, y, z\right)=\lambda_{s}(y) x \frac{\partial}{\partial x}+\lambda_{s}^{\prime}(y) x^{\prime} \frac{\partial}{\partial x^{\prime}}+\lambda_{u}(y) z \frac{\partial}{\partial z}
$$

where $\lambda_{s}(y)<\lambda_{s}^{\prime}(y)<0$ and $\lambda_{u}(y)>0$ for every $y$.

Remarks 3.4. Let $p, U,\left(x, x^{\prime}, y, z\right), \lambda_{s}(y), \lambda_{s}^{\prime}(y), \lambda_{u}(y)$ be as in the above proposition.

(i) The vector field $X$ vanishes on the one-dimensional submanifold $\left\{x=x^{\prime}=z=0\right\}$ and nowhere else. It follows that this submanifold is the intersection of the Kasner circle with $U$ :

$$
\mathcal{K} \cap U=\left\{x=x^{\prime}=z=0\right\} .
$$

(ii) For every $y$, the real numbers $\lambda_{s}(y), \lambda_{s}^{\prime}(y), \lambda_{u}(y)$ are the three non-zero eigenvalues of the derivative of $X$ at the point of coordinates $(0,0, y, 0) \in \mathcal{K}$. Recall that this derivative also has one zero eigenvalue (the corresponding eigendirection being tangent to the Kasner circle).

(iii) For every $c$, the three-dimensional sub-manifold $\{y=c\}$ is invariant under the flow of $X$ and the restriction of $X$ to this submanifold is linear.

(iv) The three sets $\{x=0\},\left\{x^{\prime}=0\right\}$ and $\{z=0\}$ are three-dimensional submanifolds, which are invariant under the flow of $X$, and contain $\mathcal{K} \cap U$. It follows that, up to permutation, these three submanifolds correspond to the submanifolds $\left\{N_{1}=0\right\},\left\{N_{2}=0\right\}$ and $\left\{N_{3}=0\right\}$. In particular,

$$
\left(\mathcal{B}_{\mathrm{VII}_{0}} \cup \mathcal{B}_{\mathrm{II}} \cup \mathcal{K}\right) \cap U=\{x=0\} \cup\left\{x^{\prime}=0\right\} \cup\{z=0\} .
$$

(v) As a consequence, the three two-dimensional submanifolds $\left\{x=x^{\prime}=0\right\},\{x=z=0\}$ and $\left\{x^{\prime}=z=0\right\}$ coincide, up to permutation, with the three two-dimensional submanifolds $\left\{N_{1}=N_{2}=0\right\},\left\{N_{1}=N_{3}=0\right\}$ and $\left\{N_{2}=N_{3}=0\right\}$. In particular,

$$
\left(\mathcal{B}_{\mathrm{II}} \cap \mathcal{K}\right) \cap U=\left\{x=x^{\prime}=0\right\} \cup\{x=z=0\} \cup\left\{x^{\prime}=z=0\right\} .
$$

(vi) The vector field $\lambda_{s}(y) x \partial / \partial x+\lambda_{s}^{\prime}(y) x^{\prime} \partial / \partial x^{\prime}+\lambda_{u}(y) z \partial / \partial z$ is not changed if we multiply the coordinates $x, x^{\prime}, y, z$ by some arbitrary real numbers. Together with item (iv), this shows that, up to multiplying $x$ and/or $x^{\prime}$ and/or $z$ by -1 , we may assume that

$$
\mathcal{B}^{+} \cap U=\left\{x \geq 0, x^{\prime} \geq 0, z \geq 0\right\} .
$$

(vii) On the one hand, since $\mathcal{K} \cap U=\left\{x=x^{\prime}=z=0\right\}$, the metric induced on $\mathcal{K} \cap U$ by the riemannian metric $d x^{2}+d x^{2}+d y^{2}+d z^{2}$ is simply $d y^{2}$. On the other hand, the vector field $\lambda_{s}(y) x \partial / \partial x+\lambda_{s}^{\prime}(y) x^{\prime} \partial / \partial x^{\prime}+\lambda_{u}(y) z \partial / \partial z$ is not changed if we replace the coordinate $y$ by $\varphi(y)$ where $\varphi: \mathbb{R} \rightarrow \mathbb{R}$ is any $C^{\infty}$ diffeomorphism such that $\varphi(0)=0$. Therefore, up to replacing $y$ by $\varphi(y)$ for some diffeomorphism $\varphi$, we may assume that the metrics induced on $\mathcal{K} \cap U$ by $d x^{2}+d x^{\prime 2}+d y^{2}+d z^{2}$ and $h=d \Sigma_{1}^{2}+d \Sigma_{2}^{2}+d \Sigma_{3}^{2}+d N_{1}^{2}+d N_{2}^{2}+d N_{3}^{2}$ coincide.

Proof of proposition 3.3. The derivative $D X(p)$ has two negative, one zero, and one positive eigenvalue. According to proposition 3.2, the vector field $X$ satisfies the Sternberg condition at $p$. Therefore, a crude 
application of Takens' theorem 2.2 implies that there exists a $C^{1}$ local coordinate system $\left(x_{1}, x_{2}, y, z\right)$ on a neighborhood $U$ of $p$ in $\mathcal{B}$, centered at $p$, such that :

$$
X\left(x_{1}, x_{2}, y, z\right)=\sum_{i=1}^{2} \sum_{j=1}^{2} a_{i, j}(y) x_{j} \frac{\partial}{\partial x_{i}}+\phi(y) \frac{\partial}{\partial y}+b(y) z \frac{\partial}{\partial z}
$$

for some real valued functions $\phi, a_{1,1}, a_{1,2}, a_{2,1}, a_{2,2}, b$ defined on a neighborhood of 0 in $\mathbb{R}$. Moreover, the eigenvalues of the matrix $\left(a_{i, j}(0)\right)$ are negative, and $b(0)$ is positive. Replacing $U$ by a smaller neighborhood of $p$ if necessary, we can assume that the three Taub points $T_{1}, T_{2}, T_{3}$ are not in $U$.

Now, (7) implies that the curve $\left\{x_{1}=x_{2}=z=0\right\}$ is the only curve in $U$ containing the point $p$, invariant under the flow of $X$, and such that $D X(p)$ vanishes on the tangent space at $p$ of this curve. Hence, the curve $\left\{x_{1}=x_{2}=z=0\right\}$ has to be the intersection of the Kasner circle $\mathcal{K}$ with $U$. Since $X$ vanishes on $\mathcal{K}$, it follows that $\phi=0$.

For $y^{0}$ small enough, let $q_{y^{0}}$ be the point of coordinates $\left(x_{1}, x_{2}, y, z\right)=\left(0,0, y^{0}, 0\right)$. This is a point of the Kasner circle $\mathcal{K}$, which is not a Taub point. Hence, the point $q_{y^{0}}$ has four distinct eigenvalues : two distinctive negative eigenvalues $\lambda_{s}\left(y^{0}\right)<\lambda_{s}^{\prime}\left(y^{0}\right)<0$, one zero eigenvalue whose associated eigendirection is tangent to the Kasner circle, and one positive eigenvalue $\lambda_{u}\left(y^{0}\right)>0$. The set $\left(y=y^{0}\right)$ is a three-dimensional manifold, transversal to the Kasner circle. Looking at (7), we see that this 3dimensional submanifold is invariant under the flow of $X$, and that the restriction of $X$ to this invariant manifold is linear.This shows that $b(c)=\lambda_{u}\left(y^{0}\right)$, and that $\lambda_{s}\left(y^{0}\right)$ and $\lambda_{s}^{\prime}\left(y^{0}\right)$ are the eigenvalues of the matrix $\left(a_{i, j}\left(y^{0}\right)\right)_{i, j=1,2}$. Since $\lambda_{s}\left(y^{0}\right)$ and $\lambda_{s}^{\prime}\left(y^{0}\right)$ are distinct, there exists a linear change of coordinates $\left(x_{1}, x_{2}, z\right) \rightarrow\left(x, x^{\prime}, z\right)$ on the submanifold $\left(y=y^{0}\right)$, so that

$$
X\left(x, x^{\prime}, y^{0}, z\right)=\lambda_{s}\left(y^{0}\right) x \frac{\partial}{\partial x}+\lambda_{s}^{\prime}\left(y^{0}\right) x^{\prime} \frac{\partial}{\partial x^{\prime}}+\lambda_{u}\left(y^{0}\right) z \frac{\partial}{\partial z} .
$$

Since eigenvalues and eigendirections of the point $q_{y^{0}}$ depend in a smooth way of $c$, one may perform the above change of coordinates simultaneously for every $y^{0}$, and get a system of $C^{\infty}$ coordinates $\left(x, x^{\prime}, y, z\right)$ defined on $U$, such that

$$
X\left(x, x^{\prime}, y, z\right)=\lambda_{s}(y) x \frac{\partial}{\partial x}+\lambda_{s}^{\prime}(y) x^{\prime} \frac{\partial}{\partial x^{\prime}}+\lambda_{u}(y) z \frac{\partial}{\partial z} .
$$

The proposition is proven.

From now on, for every point $p$ on the Kasner circle $\mathcal{K}$ which is not preperiodic under the Kasner map, we fix a $C^{1}$ local coordinate system $\left(x_{p}, x_{p}^{\prime}, y_{p}, z_{p}\right)$ on a neighborhood $U_{p}$ of $p$ in $\mathcal{B}$, centered at $p$, and such that, in this coordinate system, $X$ reads :

$$
X\left(x_{p}, x_{p}^{\prime}, y_{p}, z_{p}\right)=\lambda_{s}\left(y_{p}\right) x_{p} \frac{\partial}{\partial x_{p}}+\lambda_{s}^{\prime}\left(y_{p}\right) x_{p}^{\prime} \frac{\partial}{\partial x_{p}^{\prime}}+\lambda_{u}\left(y_{p}\right) z_{p} \frac{\partial}{\partial z_{p}}
$$

with $\lambda_{s}\left(y_{p}\right)<\lambda_{s}^{\prime}\left(y_{p}\right)<0$ and $\lambda_{u}\left(y_{p}\right)>0$ for every $y_{p}$. According to items (i), (ii), (iv) and (v) of remarks 3.4, we automatically have

$$
\begin{aligned}
\mathcal{K} \cap U_{p} & =\left\{x=x^{\prime}=z=0\right\} \\
\left(\mathcal{B}_{\mathrm{II}} \cup \mathcal{K}\right) \cap U_{p} & =\left\{x=x^{\prime}=0\right\} \cup\{x=z=0\} \cup\left\{x^{\prime}=z=0\right\}, \\
\left(\mathcal{B}_{\mathrm{VII}_{0}} \cup \mathcal{B}_{\mathrm{II}} \cup \mathcal{K}\right) \cap U_{p} & =\{x=0\} \cup\left\{x^{\prime}=0\right\} \cup\{z=0\},
\end{aligned}
$$


and $\lambda_{s}\left(y_{p}\right), \lambda_{s}^{\prime}\left(y_{p}\right), \lambda_{u}\left(y_{p}\right)$ are the three non-zero eigenvalues of the point $\left(0,0, y_{p}, 0\right) \in \mathcal{K}$. Moreover, according to items (vi) of remarks 3.4, we may — and we will — assume that

$$
\mathcal{B}^{+} \cap U_{p}=\left\{x_{p} \geq 0, x_{p}^{\prime} \geq 0, z_{p} \geq 0\right\} .
$$

We will consider the riemannian metric $g_{p}$ on $U_{p}$ defined by

$$
g_{p}:=d x_{p}^{2}+d x_{p}^{\prime 2}+d y_{p}^{2}+d z_{p}^{2} .
$$

Recall that $h$ is the riemannian metric on $\mathcal{B}$ defined by $h=d \Sigma_{1}^{2}+d \Sigma_{2}^{2}+d \Sigma_{3}^{2}+d N_{1}^{2}+d N_{2}^{2}+d N_{3}^{2}$. According to item (vii) of remarks 3.4, we may — and we will — assume that

$$
\text { the riemannian metrics induced by } g_{p} \text { and } h \text { on } \mathcal{K} \cap U_{p} \text { coincide. }
$$

For $\alpha<\beta$ and $\gamma>0$, we consider the subset $V_{p}(\alpha, \beta, \gamma)$ of $U_{p}$ defined as follows:

$$
V_{p}(\alpha, \beta, \gamma):=U_{p} \cap\left\{0 \leq x_{p}, x_{p}^{\prime}, z_{p} \leq \gamma \text { and } \alpha \leq y_{p} \leq \beta\right\} .
$$

Observe that $V_{p}$ is contained in $U_{p} \cap \mathcal{B}^{+}$. If $\alpha, \beta, \gamma$ are small enough, then $V_{p}(\alpha, \beta, \gamma)$ is contained in the interior of $U_{p}$. If $\alpha<0<\beta$, then $V_{p}(\alpha, \beta, \gamma)$ is a neighborhood of $p$ in $\mathcal{B}^{+}$.

\section{DUlaC MAP NEAR A POINT OF $\mathcal{K}$ WHICH IS NOT PREPERIODIC}

Let $p$ be a point of the Kasner circle $\mathcal{K}$ which is not preperiodic for the Kasner map. We consider the neighborhood $U_{p}$ of $p$ and the local coordinate system $\left(x_{p}, x_{p}^{\prime}, y_{p}, z_{p}\right)$ on $U_{p}$ defined at the end of the preceeding section. In particular, we assume that properties (8)...(12) and (14) are satisfied. Using (8), one can calculate explicitly the time $t$ map $X^{t}$ of the flow of the Wainwright-Hsu vector field $X$ in the $\left(x_{p}, x_{p}^{\prime}, y_{p}, z_{p}\right)$ coordinate system :

$$
X^{t}\left(x_{p}, x_{p}^{\prime}, y_{p}, z_{p}\right)=\left(x_{p} e^{\lambda_{s}\left(y_{p}\right) t}, x_{p}^{\prime} e^{\lambda_{s}^{\prime}\left(y_{p}\right) t}, y_{p}, z_{p} e^{\lambda_{u}\left(y_{p}\right) t}\right) .
$$

Now, we consider some real numbers $\alpha<\beta$ and $\gamma>0$ small enough so that the set

$$
V_{p}:=V_{p}(\alpha, \beta, \gamma)=\left\{0 \leq x_{p}, x_{p}^{\prime}, z_{p} \leq \gamma \text { and } \alpha \leq y_{p} \leq \beta\right\}
$$

is contained in the interior of $U_{p}$. The purpose of this section is to analyze quite in details the behavior of the orbits of the flow of $X$ inside $V_{p}$. In the $\left(x_{p}, x_{p}^{\prime}, y_{p}, z_{p}\right)$ coordinate system, $V_{p}$ is a 4-dimensional box, i.e. the cartesian product of four closed intervals. The boundary of $V_{p}$ is made of eight faces. Three of these eight faces will play an important role in the remainder of the paper:

$$
M_{p}:=V_{p} \cap\left\{x_{p}=\gamma\right\} \quad M_{p}^{\prime}:=V_{p} \cap\left\{x_{p}^{\prime}=\gamma\right\} \quad N_{p}:=V_{p} \cap\left\{z_{p}=\gamma\right\} .
$$

Looking at (8), we notice that $X$ is transversal to $M_{p}, M_{p}^{\prime}$ and $N_{p}$, and is tangent to the five other faces of $V_{p}$. Moreover, we notice that $X$ is pointing inward $V_{p}$ along $M_{p}$ and $M_{p}^{\prime}$; it is pointing outward $V_{p}$ along $N_{p}$. It follows that:

- an orbit of $X$ can enter in $V_{p}$ by crossing either the face $M_{p}$ or by crossing the face $M_{p}^{\prime}$;

- an orbit of $X$ can only exit $V_{p}$ by crossing the face $N_{p}$.

We need to analyze quite precisely the behavior of the orbits of $X$ inside $V_{p}$. First, we need to understand which type II orbit intersect the hypersurfaces (with boundary and corners) $M_{p}, M_{p}^{\prime}$ and $N_{p}$. Recall that, for every point $q \in \mathcal{B}_{\mathrm{II}}$, the orbit of $q$ is a heteroclinic orbit connecting two points of $\mathcal{K}$.

Proposition 4.1. Let $q$ be a point $\mathcal{B}_{I I}^{+}$, and denote by $\mathcal{O}$ the orbit of $q$. Let $\alpha(q)=\alpha(\mathcal{O})$ denote the unique $\alpha$-limit point of $\mathcal{O}$, and $\omega(q)=\omega(\mathcal{O})$ denote the unique $\omega$-limit point of $\mathcal{O}$. 
(1) The orbit $\mathcal{O}$ intersects the hypersurface $N_{p}$ if and only if the point $\alpha(q)$ is in $V_{p}$.

(2) The orbit of $\mathcal{O}$ intersects the hypersurface $M_{p} \cup M_{p}^{\prime}$ if and only if the point $\omega(q)$ is in $V_{p}$.

Proof. We prove the first statement; the second one follows from similar arguments. Of course, we will work in the $\left(x_{p}, x_{p}^{\prime}, y_{p}, z_{p}\right)$ coordinate system. According to (10), (15) and (18),

$$
\begin{aligned}
\mathcal{K} \cap V_{p} & =\left\{x_{p}=x_{p}^{\prime}=0, \alpha \leq y_{p} \leq \beta, z_{p}=0\right\}, \\
\mathcal{B}_{\mathrm{II}} \cap N_{p} & =\left\{x_{p}=x_{p}^{\prime}=0, \alpha \leq y_{p} \leq \beta, z_{p}=\gamma\right\} .
\end{aligned}
$$

Suppose that the orbit $\mathcal{O}$ intersects the hypersurface $N_{p}$ at some point $\bar{q}=(0,0, y, \gamma)$, with $\alpha \leq y \leq \beta$. Then, according to (16), the past orbit of $\bar{q}$ is contained in $V_{p}$, and converges to the point $\alpha(q)=$ $(0,0, y, 0) \in \mathcal{K} \cap V_{p}$. In particular, the point $\alpha(q)$ is in $V_{p}$. Conversely, suppose that the point $\alpha(q)$ is in $V_{p}$. Then $\alpha(q)=(0,0, y, 0)$ for some $y \in[\alpha, \beta]$. Using again (16), we see that the only orbit of $X$ in $V_{p}$ converging towards the point $(0,0, y, 0)$ as $t \rightarrow-\infty$ is the curve $t \mapsto\left(0,0, y, e^{\lambda^{u}(y) t}\right)$. Hence, the orbit $\mathcal{O}$ intersects the hypersurface $N_{p}$ at the point $\bar{q}=(0,0, y, \gamma)$.

This proposition allows to define two maps

$$
\begin{array}{rlrl}
\alpha_{p}: \mathcal{B}_{\mathrm{II}}^{+} \cap N_{p} & \longrightarrow \mathcal{K} \cap V_{p} & \omega_{p}: \mathcal{B}_{\mathrm{II}}^{+} \cap\left(M_{p} \cup M_{p}^{\prime}\right) & \longrightarrow \mathcal{K} \cap V_{p} \\
q & \longmapsto \alpha(q) & & \longmapsto \\
q & \longmapsto(q)
\end{array}
$$

The map $\alpha_{p}$ is one-to-one (there is only one type II orbit in $\mathcal{B}^{+}$which "starts" at a given point of $\mathcal{K}$ ), whereas the map $\omega_{p}$ is two-to-one (there are two type II orbits in $\mathcal{B}^{+}$which "arrive" at a given point of $\mathcal{K}$ ). The restriction of $\omega_{p}$ to $\mathcal{B}_{\mathrm{II}}^{+} \cap M_{p}$ (resp. to $\mathcal{B}_{\mathrm{II}}^{+} \cap M_{p}$ ) is one-to-one.

Proposition 4.2. The maps $\alpha_{p}$ and $\omega_{p}$ are local isometries with respect to the metrics induced on $\mathcal{B}_{I I}^{+} \cap N_{p}, \mathcal{B}_{I I}^{+} \cap\left(M_{p} \cup M_{p}^{\prime}\right)$ and $\mathcal{K} \cap V_{p}^{+}$by the riemannian metric $g_{p}=d x_{p}^{2}+d x_{p}^{\prime 2}+d y_{p}^{2}+d z_{p}^{2}$.

Proof. The proof of proposition 4.1 shows that, in the $\left(x_{p}, x_{p}^{\prime}, y_{p}, z_{p}\right)$ coordinate system, the map $\alpha_{p}$ reads $\alpha_{p}\left(0,0, y_{p}, \gamma\right)=\left(0,0, y_{p}, 0\right)$. Similarly, the map $\omega_{p}$ reads $\omega_{p}\left(\gamma, 0, y_{p}, 0\right)=\left(0,0, y_{p}, 0\right)$.

Now, we have to define and study the Dulac map associated to $V_{p}$ : this map will describe the effect of going through $V_{p}$ for the orbits of $X$.

Let $q$ be a point in $M_{p}$. If the $z_{p}(q)>0$ (which is the case if $q \in \mathcal{B}_{\text {IX }}$ ), then (16) shows that the forward orbit of $q$ will eventually exit $V_{p}$, by crossing the face $N_{p}$. If $z_{p}(q)=0$ (which is the case if $q \in \mathcal{B}_{\text {III }}$ ), then (16) shows that the forward orbit of $q$ will remain in $V_{p}$ forever. Moreover, the orbit of $q$ will converge towards the point $\omega(q) \in \mathcal{K} \cap V_{p}$. According to proposition 4.1, the type II orbit $\mathcal{O}_{\omega(q), f(\omega(q))}$ will eventually exit $V_{p}$, by crossing $N_{p}$ (since $\left.\alpha\left(\mathcal{O}_{\omega(q), f(\omega(q))}\right)=\omega(q) \in V_{p}\right)$. So, we may define a map

$$
\Phi_{p}: M_{p} \longrightarrow N_{p}
$$

as follows :

- if $z_{p}(q)>0$ then $\Phi_{p}(q)$ is the first intersection point of the orbit of $q$ with the hypersurface $N_{p}$;

- if $z_{p}(q)=0$ then $\Phi_{p}(q)$ is the first (and unique) intersection point of the type II heteroclinic orbit $\mathcal{O}_{\omega(q), f(\omega(q))}$ with the hypersurface $N_{p}$. 
We call $\Phi_{p}$ a Dulac map since it is the exact analog, in our situation, of the classical Dulac maps used to study planar vector fields. The above discussion together with formula (16) show that, in the $\left(x_{p}, x_{p}^{\prime}, y_{p}, z_{p}\right)$ coordinate system, the map $\Phi_{p}$ reads :

$$
\begin{aligned}
\Phi_{p}\left(1, x_{p}^{\prime}, y_{p}, z_{p}\right) & =\left(z_{p}^{-\lambda_{s}\left(y_{p}\right) / \lambda_{u}\left(y_{p}\right)}, x_{p}^{\prime} . z_{p}^{-\lambda_{s}^{\prime}\left(y_{p}\right) / \lambda_{u}\left(y_{p}\right)}, y_{p}, 1\right) \text { if } z_{p}>0 \\
\Phi_{p}\left(1, x_{p}^{\prime}, y_{p}, 0\right) & =\left(0,0, y_{p}, 1\right)
\end{aligned}
$$

For every $q \in M_{p}$, we decompose $T_{q} M_{p}$ as a direct sum of two linear subspaces $F_{q}^{s} \oplus F_{q}^{u}$ where

$$
\begin{aligned}
F_{q}^{s} & :=\mathbb{R} \cdot \frac{\partial}{\partial x_{p}^{\prime}}(q) \oplus \mathbb{R} \cdot \frac{\partial}{\partial z_{p}}(q) \\
F_{q}^{u} & :=\mathbb{R} \cdot \frac{\partial}{\partial y_{p}}(q) .
\end{aligned}
$$

Similarly, for every $r \in N_{p}$, we write $T_{r} N_{p}$ as a direct sum of two linear subspaces $G_{r}^{s} \oplus F_{r}^{u}$ where

$$
\begin{aligned}
G_{r}^{s} & :=\mathbb{R} \cdot \frac{\partial}{\partial x_{p}}(r) \oplus \mathbb{R} \cdot \frac{\partial}{\partial x_{p}^{\prime}}(r) \\
G_{r}^{u} & :=\mathbb{R} \cdot \frac{\partial}{\partial y_{p}}(r) .
\end{aligned}
$$

We are now ready to state the properties of the Dulac map $\Phi_{p}$ which are the core of the proof of our main theorem 1.3:

Proposition 4.3. The Dulac map $\Phi_{p}: M_{p} \rightarrow N_{p}$ is $C^{1}$. Moreover, for every point $q \in M_{p} \cap\left\{z_{p}=0\right\}$, the derivative $D \Phi_{p}(q): T_{q} M_{p} \rightarrow T_{\Phi_{p}(q)} N_{p}$ of the map $\Phi_{p}$ at $q$ satisfies:

- $D \Phi_{p}(q) \cdot v=0$ for every vector $v \in F_{q}^{s}$;

- $D \Phi_{p}(q)$ maps $F_{q}^{u}$ on $G_{\Phi_{p}(q)}^{u}$, and $\left\|D \Phi_{p}(q) \cdot v\right\|_{g_{p}}=\|v\|_{g_{p}}$ for every vector $v \in F_{q}^{u}$.

This proposition roughly says the following: when an orbit of $X$ passes close to the point $p \in \mathcal{K}$, the distance from this orbit to the mixmaster attractor $\mathcal{A}=\mathcal{K} \cup \mathcal{B}_{\mathrm{II}}$ is contracted super-linearly (in the $\left(x_{p}, x_{p}^{\prime}, y_{p}, z_{p}\right)$ coordinates system, this distance in measured by the $x_{p}, x_{p}^{\prime}, z_{p}$ coordinates), whereas there is no drift in the direction tangent to the attractor (in the $\left(x_{p}, x_{p}^{\prime}, y_{p}, z_{p}\right)$ coordinates system, this drift is measured by the $y_{p}$ coordinate).

Proof of proposition 4.3. The fact that $\Phi_{p}$ is $C^{1}$ (and even analytical) in restriction to $M_{p} \cap\left\{z_{p}>0\right\}$ is an immediate consequence of formula (19). The fact that $\Phi_{p}$ is $C^{1}$ on $M_{p} \cap\left\{z_{p}=0\right\}$ follows from (19) and (20), together with the inequalities

$$
-\frac{\lambda_{s}\left(y_{p}\right)}{\lambda_{u}\left(y_{p}\right)}>1 \quad \text { and } \quad-\frac{\lambda_{s}^{\prime}\left(y_{p}\right)}{\lambda_{u}\left(y_{p}\right)}>1
$$

(see proposition 3.1 $)^{1}$. The same ingredients actually show that, for every point $q \in M_{p} \cap\left\{z_{p}=0\right\}$,

$$
D \Phi_{p}(q) \cdot \frac{\partial}{\partial x_{p}^{\prime}}(q)=D \Phi_{p}(q) \cdot \frac{\partial}{\partial z_{p}}(q)=0 \quad \text { and } D \Phi_{p}(q) \cdot \frac{\partial}{\partial y_{p}}(q)=\frac{\partial}{\partial y_{p}}(\Phi(q)) .
$$

\footnotetext{
${ }^{1}$ We do not know if $\Phi_{p}$ is $C^{1+\varepsilon}$ for any $\varepsilon>0$, unless we have some better estimates on the ratios $\frac{\lambda_{s}\left(y_{p}\right)}{\lambda_{u}\left(y_{p}\right)}$ and $\frac{\lambda_{s}^{\prime}\left(y_{p}\right)}{\lambda_{u}\left(y_{p}\right)}$.
} 
The proposition follows.

The coordinate $x_{p}$ and $x_{p}^{\prime}$ play similar roles. So, we will also consider the map

$$
\Phi_{p}^{\prime}: M_{p}^{\prime} \longrightarrow N_{p}
$$

defined as follows :

- if $z_{p}(q)>0$ then $\Phi_{p}^{\prime}(q)$ is the first intersection point of the orbit of $q$ with the hypersurface $N_{p}$;

- if $z_{p}(q)=0$ then $\Phi_{p}^{\prime}(q)$ is the first (and unique) intersection point of the type II heteroclinic orbit $\mathcal{O}_{\omega(q), f(\omega(q))}$ with the hypersurface $N_{p}$.

In the $\left(x_{p}, x_{p}^{\prime}, y_{p}, z_{p}\right)$ coordinate system, the map $\Phi_{p}^{\prime}$ reads :

$$
\begin{aligned}
\Phi_{p}^{\prime}\left(x_{p}, 1, y_{p}, z_{p}\right) & =\left(x_{p} . z_{p}^{-\lambda_{s}\left(y_{p}\right) / \lambda_{u}\left(y_{p}\right)}, z_{p}^{-\lambda_{s}^{\prime}\left(y_{p}\right) / \lambda_{u}\left(y_{p}\right)}, y_{p}, 1\right) \text { if } z_{p}>0 \\
\Phi_{p}^{\prime}\left(x_{p}, 1, y_{p}, 0\right) & =\left(0,0, y_{p}, 1\right)
\end{aligned}
$$

For every $q \in M_{p}^{\prime}$, we will write $T_{q} M_{p}^{\prime}$ as a direct sum of two linear subspaces $F_{q}^{s} \oplus F_{q}^{u}$ where

$$
\begin{aligned}
F_{q}^{s} & :=\mathbb{R} \cdot \frac{\partial}{\partial x_{p}}(q) \oplus \mathbb{R} \cdot \frac{\partial}{\partial z_{p}}(q) \\
F_{q}^{u} & :=\mathbb{R} \cdot \frac{\partial}{\partial y_{p}}(q) .
\end{aligned}
$$

Then, we can summarize the key properties of the map $\Phi_{p}^{\prime}$ as follow :

Proposition 4.4. The map $\Phi_{p}^{\prime}: M_{p}^{\prime} \rightarrow N_{p}$ is $C^{1}$. Moreover, for every $q \in M_{p}^{\prime} \cap\left\{z_{p}=0\right\}$, the derivative of $D \Phi_{p}^{\prime}(q): T_{q} M_{p}^{\prime} \rightarrow T_{\Phi_{p}^{\prime}(q)} N_{p}$ of the map $\Phi_{p}^{\prime}$ at $q$ satisfies :

- $D \Phi_{p}^{\prime}(q) \cdot v=0$ for every vector $v \in F_{q}^{s}$;

- $D \Phi_{p}^{\prime}(q)$ maps $F_{q}^{u}$ on $G_{\Phi_{p}^{\prime}(q)}^{u}$, and $\left\|D \Phi_{p}^{\prime}(q) \cdot v\right\|_{g_{p}}=\|v\|_{g_{p}}$ for every vector $v$ in $F_{q}^{u}$.

\section{CONSTRUCTION OF A LOCAL SECTION FOR A CLOSED $f$-INVARIANT APERIODiC SET OF $\mathcal{K}$}

From now on until the end of section 7, we consider a closed, $f$-invariant, aperiodic subset $C$ of the Kasner circle $\mathcal{K}$.

Observe that $C$ is necessarly totally discontinuous. Indeed the points of $\mathcal{K}$ that are preperiodic for the Kasner map $f$ are dense in $\mathcal{K}$ : this can be proved in several different ways; this follows for example the equivalence $2 \Leftrightarrow 3$ of proposition 3.2. Let $\mathcal{C}$ be the union of $C$ and of all the type II orbits connecting two points of $C$ :

$$
\mathcal{C}:=C \cup \bigcup_{q \in C} \mathcal{O}_{q, f(q)}
$$


We want to prove that, for every point $q \in C$, the stable manifold $W^{s}(q)$ contains a 3-dimensional disc (see definition 1.2 and theorem 1.3). To this end, we will consider a kind of "Poincare section" for $\mathcal{C}$, and study the Poincaré map associated with this section ${ }^{2}$.

For every $p \in C$, we consider the neighborhood $U_{p}$ of $p$, and the local coordinate system $\left(x_{p}, x_{p}^{\prime}, y_{p}, z_{p}\right)$ on $U_{p}$ defined in section 3 . Recall that, for every $\alpha, \beta, \gamma$, we denote

$$
V_{p}(\alpha, \beta, \gamma):=U_{p} \cap\left\{0 \leq x_{p}, x_{p}^{\prime}, y_{p} \leq \gamma \text { and } \alpha \leq y_{p} \leq \beta\right\} .
$$

Recall that $V_{p}(\alpha, \beta, \gamma)$ is a neighborhood of $p$ in $\mathcal{B}^{+}$provided that $\alpha<0<\beta$ and $\gamma>0$. For each $p \in C$, we choose $\alpha_{p}^{0}<0<\beta_{p}^{0}$ and $\gamma_{p}^{0}>0$ small enough, so that

$$
V_{p}^{0}:=V_{p}\left(\alpha_{p}^{0}, \beta_{p}^{0}, \gamma_{p}^{0}\right)
$$

is contained in the interior of $U_{p}$. Up to slightly modifying $\alpha_{p}^{0}, \beta_{p}^{0}, \gamma_{p}^{0}$ we can assume that the boundary of $V_{p}^{0}$ is disjoint from $C$ (that is, the points of coordinates $\left(x_{p}, x_{p}^{\prime}, y_{p}, z_{p}\right)=(0,0, \alpha, 0)$ and $\left(x_{p}, x_{p}^{\prime}, y_{p}, z_{p}\right)=(0,0, \beta, 0)$ are not in $\left.C\right)$ : this is possible since $C$ is totally discontinuous.

Since $C$ is compact, one can find a finite number of points $p_{1}, \ldots, p_{n} \in C$ such that the neighborhoods $V_{p_{1}}^{0}, \ldots, V_{p_{n}}^{0}$ cover $C$. Now, we modify these neighborhoods in order to make them pairwise disjoint:

- we set $\left(\alpha_{p_{1}}, \beta_{p_{1}}, \gamma_{p_{1}}\right):=\left(\alpha_{p_{1}}^{0}, \beta_{p_{1}}^{0}, \gamma_{p_{1}}^{0}\right)$, and $V_{p_{1}}:=V_{p_{1}}\left(\alpha_{p_{1}}, \beta_{p_{1}}, \gamma_{p_{1}}\right)=V_{p_{1}}^{0}$;

- then, we can find some constants $\alpha_{2}, \beta_{2}, \gamma_{2}$ such that $V_{p_{2}}:=V_{p_{2}}\left(\alpha_{2}, \beta_{2}, \gamma_{2}\right)$ is contained in $V_{p_{2}}^{0} \backslash V_{p_{1}}$, and such that $C \cap\left(V_{p_{2}}^{0} \backslash V_{p_{1}}\right)$ is contained in the interior of $V_{p_{2}}$;

- then, we can find some constants $\alpha_{3}, \beta_{3}, \gamma_{3}$ such that $V_{p_{3}}:=V_{p_{3}}\left(\alpha_{3}, \beta_{3}, \gamma_{3}\right)$ is contained in $V_{p_{3}}^{0} \backslash\left(V_{p_{1}} \cup V_{p_{2}}\right)$, and such that $C \cap\left(V_{p_{3}}^{0} \backslash\left(V_{p_{1}} \cup V_{p_{2}}\right)\right)$ is contained in the interior of $V_{p_{3}}$;

- etc.

At the end of this process, we get $n$ pairwise disjoint domains $V_{p_{1}}, \ldots, V_{p_{n}}$, such that $C$ is contained in the interior of $V_{p_{1}} \cup \cdots \cup V_{p_{n}}$. For each $i$, there are some constants $\alpha_{i}, \beta_{i}, \gamma_{i}$ such that $V_{p_{i}}=V_{p_{i}}^{\alpha_{i}, \beta_{i}, \gamma_{i}}$, and $V_{p_{i}}$ is contained in the interior of $U_{p_{i}}$. Hence, the result of section 4 apply to $V_{p_{i}}$. It may happen that, for some $i$, the point $p_{i}$ is not in $V_{p_{i}}$ (i.e. that $\alpha_{i}$ or $\beta_{i}$ is non-positive), but we do not care.

Now, we denote

$V:=V_{p_{1}} \sqcup \cdots \sqcup V_{p_{n}} \quad M:=M_{p_{1}} \sqcup \cdots \sqcup M_{p_{n}} \quad M^{\prime}:=M_{p_{1}}^{\prime} \sqcup \cdots \sqcup M_{p_{n}}^{\prime} \quad N:=N_{p_{1}} \sqcup \cdots \sqcup N_{p_{n}}$.

Then $V$ is a neighborhood of $C$ in $\mathcal{B}^{+}$. The hypersurfaces $M, M^{\prime}$ and $N$ are transverse to $X$. An orbit of $X$ can only enter in $V$ by crossing $M \cup M^{\prime}$, and can only exit $V$ by crossing $N$. Moreover, according to proposition 4.1, we have the following important properties :

Proposition 5.1. Every type II orbit whose w-limit point is in $C$ intersects $M \cup M^{\prime}$. Every type II orbit whose $\alpha$-limit point is in $C$ intersects $N$.

We will see $M \cup M^{\prime}$ as a kind of "Poincaré section" for $\mathcal{C}$. Let us define the "Poincaré map" $\Theta$ associated to this section. First, we consider the "Dulac map"

$$
\Phi: M \cup M^{\prime} \rightarrow N
$$

\footnotetext{
${ }^{2}$ The set $\mathcal{C}$ cannot admit a true Poincaré section, since it contains some singularities of $X$ (namely, the points of $C$ ). Nevertheless, we will consider a hypersurface with boundary $N$ so that every type II orbit in $\mathcal{C}$ intersects $N$ transversally. The hypersurface $N$ will play the role of a Poincaré section.
} 
defined by $\Phi_{\mid M_{p_{i}}}=\Phi_{p_{i}}$ and $\Phi_{\mid M_{p_{i}}^{\prime}}=\Phi_{p_{i}}^{\prime}$. For $q \in M \cup M^{\prime}$ :

- if the forward of orbit of $q$ exits $V$ by crossing $N$ (which is the case if $q \in \mathcal{B}_{\mathrm{IX}}$ ), then $\Phi(q)$ is by definition the first intersection point of the orbit of $q$ with the hypersurface $N$;

- if the forward orbit of $q$ remains in $V$ forever (which is the case for every $q \in \mathcal{B}_{\mathrm{II}}$ ), then $\Phi(q)$ is the first intersection point of the type II heteroclinic orbit $\mathcal{O}_{\omega(q), f(\omega(q))}$ with the hypersurface $N$.

Now, we consider the "transition map"

$$
\Psi: N \rightarrow M \cup M^{\prime}
$$

partially defined as follows: if the forward orbit of $q$ re-enters in $V$ by crossing the hypersurface $M \cup M^{\prime}$, then $\Psi(q)$ is the first intersection point of this forward orbit of $q$ with $M \cup M^{\prime}$; otherwise $\Psi(q)$ is not defined. The "Poincaré map"

$$
\Theta: M \cup M^{\prime} \rightarrow M \cup M^{\prime}
$$

is by definition the product of the "Dulac map" $\Phi$ and the "transition map" $\Psi$.

In the next section, we will study the dynamics Poincaré map $\Theta$. For this purpose, we will use a riemannian metric on $g$ on $\mathcal{B}$ such that, for $i=1, \ldots, n$,

$$
g_{\mid V_{i}}=g_{p_{i}}=d x_{p_{i}}^{2}+d x_{p_{i}}^{\prime 2}+d y_{p_{i}}^{2}+d z_{p_{i}}^{2} .
$$

\section{Existence of Stable MANifolds For the Poincaré map $\Theta: M \cup M^{\prime} \rightarrow M \cup M^{\prime}$}

The purpose of this section is to prove that, for every point $q \in \mathcal{C} \cap\left(M \cup M^{\prime}\right)$, the stable manifold of $q$ for the "Poincaré map" $\Theta$ contains a two-dimensional disc in $M \cup M^{\prime}$. To this end, we will prove that $\mathcal{C} \cap\left(M \cup M^{\prime}\right)$ is a hyperbolic set for the map $\Theta$, and we will use a classical results on stable manifolds for hyperbolic sets.

Definition 6.1. Let $(\mathcal{M}, g)$ be a riemannian manifold and $\phi: \mathcal{M} \rightarrow \mathcal{M}$ be a $C^{1}$ map. A hyperbolic set for the map $\phi$ is a compact $\phi$-invariant subset $C$ of $\mathcal{M}$ such that, for every $q \in C$, there is splitting $T_{q} \mathcal{M}=F_{q}^{s} \oplus F_{q}^{u}$ which depends continuously on $q$ and such that, for some constant $\mu, \nu \in(0,1)$ :

$$
\begin{aligned}
& D \phi(q) . F_{q}^{s} \subset F_{\phi(q)}^{s} \text { and }\|D \phi(x) \cdot v\| \leq \mu\|v\| \text { for every } q \in C \text { and } v \in F_{q}^{s} \\
& D \phi(q) . F_{q}^{u}=F_{\phi(q)}^{u} \text { and }\left\|(D \phi(q))^{-1} \cdot v\right\| \leq \nu\|v\| \text { for every } q \in C \text { and } v \in F_{q}^{u} .
\end{aligned}
$$

The dimension of the vector space $F_{q}^{s}$ is called the index of $C$. The constant $\mu$ is called a contraction rate of $\phi$ on $C$.

Theorem 6.2. (see e.g. [6, page 167]) Let $\phi: \mathcal{M} \rightarrow \mathcal{M}$ be a $C^{1}$ map of a manifold $\mathcal{M}$, and $C$ be a compact subset of $\mathcal{M}$ which is a hyperbolic of index s for the map $\phi$. Then, for every $\varepsilon$ small enough, for every $x \in C$, the set

$$
W_{\varepsilon}^{s}(x):=\left\{y \in \mathcal{M} \mid \operatorname{dist}\left(\phi^{n}(y), \phi^{n}(x)\right) \leq \varepsilon \text { for every } n \geq 0\right\}
$$

is a $C^{1}$-disc of dimension s embedded in $\mathcal{M}$, tangent to $F_{x}^{s}$ at $x$, depending continuously on $x$ (in the $C^{1}$ topology). Moreover, if $\mu$ is a contraction constant for $\phi$ on $C$, then there exists a constant $\kappa$ such that, for every $\varepsilon$ small enough, for every $q \in C$ and every $r \in W_{\varepsilon}^{s}(q)$,

$$
\operatorname{dist}_{g}\left(\phi^{n}(r), \phi^{n}(q)\right) \leq \kappa \mu^{n} \text {. }
$$


We want to apply this theorem to the "Poincaré map" $\Theta: M \cup M^{\prime} \rightarrow M \cup M^{\prime}$. So we need to prove that $\mathcal{C} \cap\left(M \cup M^{\prime}\right)$ is a hyperbolic set for $\Theta$. Recall that $M \cup M^{\prime}=M_{p_{1}} \sqcup \cdots \sqcup M_{p_{n}} \sqcup M_{p_{1}}^{\prime} \sqcup \cdots \sqcup M_{p_{n}}^{\prime}$. So, for every $q \in M \cup M^{\prime}$, we have already defined a splitting $T_{q} M \cup M^{\prime}=F_{q}^{s} \oplus F_{p}^{u}$ in section 4. It remains to prove that $\Theta$ satisfies (29) and (30) with respect to these splitting. For this purpose, we will use the decomposition of $\Theta$ as a product :

$$
\Theta=\Psi \circ \Phi .
$$

The behavior of the derivative of "Dulac map" $\Phi$ was already studied in section 4 ; more precisely, we can rephrase propositions 4.3 and 4.4 as follows :

Proposition 6.3. The map $\Phi: M \cup M^{\prime} \rightarrow N$ is $C^{1}$. Moreover, for every $q \in \mathcal{C} \cap\left(M \cup M^{\prime}\right)$, the derivative of $D \Phi(q): T_{q}\left(M \cup M^{\prime}\right) \rightarrow T_{\Phi(q)} N$ of the map $\Phi$ at $q$ satisfies :

- $D \Phi(q) . v=0$ for every vector $v \in F_{q}^{s}$;

- $D \Phi(q)$ maps $F_{q}^{u}$ on $G_{\Phi(q)}^{u}$, and $\|D \Phi(q) . v\|_{g_{p}}=\|v\|_{g_{p}}$ for every vector $v$ in $F_{q}^{u}$.

It remains to study the behavior of the derivative of the "transition map" $\Psi: N \rightarrow M \cup M^{\prime}$. We recall that $\Psi(q)$ is well-defined only if the forward orbit of $q$ intersects $M \cup M^{\prime}$. So our first task is to show that $\Psi$ is well-defined at least on a neighborhood of $\mathcal{C} \cap N$ in $N$.

Proposition 6.4. There exists a neighborhood $\mathcal{V}$ of $\mathcal{C} \cap N$ in $N$, such that, for every $q \in \mathcal{V}$, the orbit of $q$ intersects $M \cup M^{\prime}$ after some time $t(q)$ which depends in a $C^{1}$ way on $q$. The map $\Psi$ is welldefined and $C^{1}$ on $\mathcal{V}$. Moreover, there exists $\nu>1$ such that, for every $q \in \mathcal{C} \cap N$, the derivative $D \Psi(q): T_{q} N \rightarrow T_{\Psi(q)} M$ satisfies

- $D \Psi(q) \cdot G_{q}^{u}=F_{\Psi(q)}^{u}$ and $\|D \Psi(q) \cdot v\|_{g} \geq \nu\|v\|_{g}$ for every $v \in G_{q}^{u}$.

Proof. Consider a point $q \in \mathcal{C} \cap N$. By proposition 5.1, the orbit of $q$ intersects $M \cup M^{\prime}$ at some point $r \in \mathcal{C} \cap\left(M \cup M^{\prime}\right)$. Now, recall that :

- $N$ and $M \cup M^{\prime}$ are $C^{1}$ hypersurfaces with boundary that are transversal to the orbits of $X$;

- $V$ was chosen so that $C$ is contained in the interior of $V$. This implies that $\mathcal{C}$ does not intersect neither the boundary of the hypersurface $N$, nor the boundary of hypersurface $M \cup M^{\prime}$. Hence, $q$ is in the interior of $N$, and $r$ is in the interior of $M \cup M^{\prime}$.

This implies the existence of a neighborhood $\mathcal{V}_{q}$ of $q$ in $N$ such that, for every $q^{\prime} \in \mathcal{V}$, the forward orbit of $q^{\prime}$ intersects $M \cup M^{\prime}$ after some time $t\left(q^{\prime}\right)$ which depends in a $C^{1}$ way on $q^{\prime}$. By definition of $\Psi$, for every $q^{\prime} \in \mathcal{V}_{q}$, we have $\Psi\left(q^{\prime}\right):=X^{t\left(q^{\prime}\right)}\left(q^{\prime}\right)$. In particular, $\Psi$ is well-defined and $C^{1}$ on $\mathcal{V}_{q}$. This proves the two first statements of the proposition.

Since $\mathcal{B}_{\mathrm{II}}$ is invariant under the flow of $X$, the map $\Psi$ maps $\mathcal{B}_{\mathrm{II}} \cap N$ on $\mathcal{B}_{\mathrm{II}} \cap\left(M \cup M^{\prime}\right)$. Now, observe that, for every $q \in \mathcal{B}_{\mathrm{II}} \cap N$, the direction $G_{q}^{u}$ is nothing but the tangent space of $\mathcal{B}_{\mathrm{II}} \cap N$ at $q$, and the direction $F_{\Psi(q)}^{u}$ is nothing but the tangent space of $\mathcal{B}_{\mathrm{II}} \cap\left(M \cup M^{\prime}\right)$ at $\Psi(q)$. This shows that $d \Psi(q)$ maps $G_{g}^{u}$ on $F_{\Psi(q)}^{u}$ for every $q \in \mathcal{C} \cap N$.

We are left to $\nu>1$ such that $\|D \Psi(q) . v\|_{g} \geq \nu\|v\|_{g}$ for every for every $q \in \mathcal{C} \cap N$ and every $v \in G_{q}^{u}$. For this purpose, we will use the maps

$$
\alpha: \mathcal{B}_{\mathrm{II}} \cap N \longrightarrow \mathcal{K} \cap V \quad \text { and } \quad \omega: \mathcal{B}_{\mathrm{II}} \cap N \longrightarrow \mathcal{K} \cap V .
$$


We recall that $\alpha$ maps a point $r \in \mathcal{B}_{\mathrm{II}} \cap N$ to the $\alpha$-limit point of the orbit of $r$, and that $\omega$ maps a point $s \in \mathcal{B}_{\mathrm{II}} \cap\left(M \cup M^{\prime}\right)$ to the $\omega$-limit point of the orbit of $s$ (see section 4). We also recall that $\alpha$ is a $C^{1}$ local isometry for the metrics induced by $g$ on $\mathcal{B}_{\mathrm{II}} \cap N$ and $\mathcal{K} \cap V$, and that $\omega$ is a $C^{1}$ local isometry for the metrics induced by $g$ on $\mathcal{B}_{\mathrm{II}} \cap\left(M \cup M^{\prime}\right)$ and $\mathcal{K} \cap V$ (proposition 4.2). Finally, we observe that, for $r \in \mathcal{B}_{\mathrm{II}} \cap N$,

$$
\omega(\Psi(r))=\omega(r)=f(\alpha(r)) .
$$

The first equality is due to the fact that $\Psi(r)$ and $r$ are on the same orbit ; the second one is an immediate consequence of the definition of the Kasner map $f$. This shows that the last statement of proposition 6.4 is equivalent to the following statement about the Kasner map : there exists a constant $\nu>1$ such that, for every $p \in C$ and every $v \in T_{p} \mathcal{K}$, one has $\|D f(p) . v\|_{g} \geq \nu .|v|_{g}$.

This last statement is an immediate consequence of the elementary properties of the Kasner map, and of our choice of the riemannian metric $g$. Indeed, the riemannian metric $g$ was chosen so that it induces the same metric on $\mathcal{K} \cap V$ as the euclidean metric $h=d \Sigma_{1}^{2}+d \Sigma_{2}^{2}+d \Sigma_{3}^{2}+d N_{1}^{2}+d N_{2}^{2}+d N_{3}^{2}$ (see (14) at the end of section 3 ). And, since $C$ is a compact subset of the Kasner circle $\mathcal{K}$ which does not contain any of the three special points $T_{1}, T_{2}, T_{3}$, there exists a constant $\nu_{C}>1$ such that, for every $q \in C$ and every $v \in T_{p} \mathcal{K}$, we have $\|D f(p) . v\|_{h} \geq \nu_{C}\|v\|$, where $\|\cdot\|_{h}$ denotes the metric induced on $\mathcal{K}$ by the euclidean metric $h$.

Let $\mathcal{U}:=\Phi^{-1}(\mathcal{V})$. Clearly, $\mathcal{U}$ is a neighborhood of $\mathcal{C} \cap\left(M \cup M^{\prime}\right)$ in $M \cup M^{\prime}$. Combining propositions 6.3 and 6.4, one immediately gets :

Proposition 6.5. The "Poincaré map" $\Theta$ is well-defined and $C^{1}$ on $\mathcal{U}$. The compact set $\mathcal{C} \cap\left(M \cup M^{\prime}\right)$ is a hyperbolic set for $\Theta$. More precisely, there exists a constant $\nu \in(0,1)$ such that, for every $q \in$ $\mathcal{C} \cap\left(M \cup M^{\prime}\right)$,

- $d \Theta(q) \cdot v=0$ for every $v \in F_{q}^{s}$,

- $d \Theta(q) \cdot F_{q}^{u}=F_{\Theta(q)}^{u}$, and $\left\|(d \Theta(q))^{-1} . v\right\|_{g}<\nu\|v\|_{g}$ for every $v \in F_{\Theta}^{u}(q)$.

Proposition 6.5 shows that the map $\Theta: M \cup M^{\prime} \rightarrow M \cup M^{\prime}$ and the $\operatorname{set} \mathcal{C} \cap M$ satisfy the hypotheses of the stable manifold theorem 6.2 (for any contraction rate $\mu>0$ ). This shows the existence of local stable manifold, with respect to the map $\Theta$, for the points of $\mathcal{C} \cap\left(M \cup M^{\prime}\right)$ :

Theorem 6.6. For every $\varepsilon$ small enough, for every $q \in \mathcal{C} \cap\left(M \cup M^{\prime}\right)$, the set

$$
W_{\varepsilon}^{s}(q):=\left\{r \in M \cup M^{\prime} \mid \text { dist }_{g}\left(\Theta^{n}(r), \Theta^{n}(q)\right) \leq \varepsilon \text { for every } n \geq 0\right\}
$$

is a $C^{1}$-embedded disc of dimension 2 in $M \cup M^{\prime}$, tangent to $F_{q}^{s}$ at $q$, depending continuously on $q$ (in the $C^{1}$ topology). For every constant $\mu>0$, there exists another constant $K$ such that, for every $q \in \mathcal{C} \cap\left(M \cup M^{\prime}\right)$, for every $r \in W_{\varepsilon}^{s}(q)$ and every $n \geq 0$

$$
\operatorname{dist}_{g}\left(\Theta^{n}(r), \Theta^{n}(q)\right) \leq K \mu^{n} \text {. }
$$

\section{STABLE MANIFOLDS FOR THE FLOW}

We are left to prove that theorem 6.6 implies our main theorem 1.3.

Consider a point $q \in C$. Then heteroclinic orbit $\mathcal{O}_{q, f(q)}$ intersects the "Poincaré section" $M \cup M^{\prime}$ at one and only one point, that we denote by $\bar{q}$. Note that $\bar{q} \in \mathcal{C} \cap\left(M \cup M^{\prime}\right)$. The set $W_{\varepsilon}^{s}(\Theta, \bar{q})$ 
defined in the statement of theorem 6.6 is a $C^{1}$-embedded two-dimensional disc in the three-dimensional hypersurface with boundary $M \cup M^{\prime}$. This disc is tangent to $F_{\bar{q}}^{s}$ at $\bar{q}$. Since the two-dimensional submanifold $\mathcal{B}_{\mathrm{VII}_{0}} \cup \mathcal{B}_{\mathrm{II}}$ is not tangent to $F_{\bar{q}}^{s}$ at $\bar{q}$, this implies that $W_{\varepsilon}^{s}(\Theta, \bar{q}) \cap \mathcal{B}_{\text {IX }}$ contains a $C^{1}$ embedded two-dimensional disc in $M \cup M^{\prime}$. Moreover, this disc depends continuously on $\bar{q}$. Consider a point $r \in W_{\varepsilon}^{s}(\Theta, \bar{q}) \cap \mathcal{B}_{\mathrm{IX}}$.

Claim 1. There is an increasing sequence of times $\left(t_{n}\right)_{n \geq 0}$ such that dist $t_{g}\left(X^{t_{n}}(r), f^{n}(q)\right) \underset{n \rightarrow \infty}{\longrightarrow} 0$.

According to theorem 6.6, we have

$$
\operatorname{dist}_{g}\left(\Theta^{n}(r), \Theta^{n}(\bar{q})\right) \underset{n \rightarrow \infty}{\longrightarrow} 0 .
$$

Together with the continuity of the flow of $X$, this shows the existence of a increasing sequence of real numbers $\left(\tau_{n}\right)_{n \geq 0}$ such that

$$
\operatorname{dist}_{g}\left(X^{\tau_{n}}\left(\Theta^{n}(r)\right), \omega\left(\Theta^{n}(\bar{q})\right)\right) \underset{n \rightarrow \infty}{\longrightarrow} 0 .
$$

Since $r \in \mathcal{B}_{\text {IX }}$, there exists an increasing sequence of times $\left(\theta_{n}\right)_{n \geq 0}$ such that, for every $n \geq 0$,

$$
\Theta^{n}(r)=X^{\theta_{n}}(r)
$$

Since $\bar{q} \in \mathcal{B}_{\mathrm{II}}$, we have, for every $n \geq 0$,

$$
\omega\left(\Theta^{n}(\bar{q})\right)=f^{n}(\omega(\bar{q}))=f^{n+1}(q) .
$$

For every $n \geq 0$, let $t_{n+1}:=\tau_{n}+\theta_{n}$. Then $\left(t_{n}\right)_{n \geq 0}$ is an increasing sequence, and

$$
\operatorname{dist}_{g}\left(X^{t_{n+1}}(r), f^{n+1}(q)\right) \underset{n \rightarrow \infty}{\longrightarrow} 0 .
$$

Claim 2. The Hausdorff distance between the piece of orbit $\left\{X^{t}(r) ; t_{n} \leq t \leq t_{n+1}\right\}$ and the heteroclinic orbit $\mathcal{O}_{f^{n}(q), f^{n+1}(q)}$ tends to 0 when $n$ goes to $+\infty$.

To prove this claim, we decompose the piece of orbit $\left\{X^{t}(r) ; t_{n} \leq t \leq t_{n+1}\right\}$ into three sub-pieces:

- First, the piece of orbit going from $X^{t_{n}}\left(r_{0}\right)$ to $\Phi\left(\Theta^{n}(r)\right)$, contained in $V$. Formula (16), together with (35) shows that, for $n$ large, this piece of orbit is close to the heteroclinic orbit $\mathcal{O}_{f^{n}(q), f^{n+1}(q)}$. In particular, for $n$ large, the point $\Phi\left(\Theta^{n}(r)\right)$ is close to the heteroclinic orbit $\mathcal{O}_{f^{n}(q), f^{n+1}(q)}$.

- Then, a piece of orbit going from $\Phi\left(\Theta^{n}(r)\right)$ to $\Theta^{n+1}(r)$, contained in $\mathcal{B} \backslash V$. For $n$ large, this piece of orbit is close to the heteroclinic orbit $\mathcal{O}_{f^{n}(q), f^{n+1}(q)}$. Indeed, for $n$ large, the point $\Phi\left(\Theta^{n}\left(r_{0}\right)\right)$ is close to the heteroclinic orbit $\mathcal{O}_{f^{n}(q), f^{n+1}(q)}$, and if we write $\Theta^{n+1}(r)=X^{t\left(\Phi\left(\Theta^{n}(r)\right)\right)}\left(\Phi\left(\Theta^{n}(r)\right)\right)$, then $t\left(\Phi\left(\Theta^{n}(r)\right)\right)$ depends continuously on $\Phi\left(\Theta^{n}(r)\right)$ (proposition 6.4) and thus is uniformly bounded.

- Finally, a piece of orbit going from $\Theta^{n+1}(r)$ to $X^{t_{n+1}}(r)$, contained in $V$. Formula (16) together with (35) show that, for $n$ large, this piece of orbit is close to the heteroclinic orbit $\mathcal{O}_{f^{n}(q), f^{n+1}(q)}$.

Now, we fix $\eta>0$, and we set

$$
D_{\mathrm{IX}}^{s}(q):=\bigcup_{-\eta \leq t \leq \eta} X^{t}\left(W_{\varepsilon}^{s}(\Theta, \bar{q}) \cap \mathcal{B}_{\mathrm{IX}}\right) .
$$


Since $\left.W_{\varepsilon}^{s}(\Theta, \bar{q})\right) \cap \mathcal{B}_{\text {IX }}$ is a $C^{1}$-embedded 2-dimensional disc in $M$, and since the orbits of $X$ are transversal to $M$, we get that $D_{\mathrm{IX}}^{s}(q)$ is a $C^{1}$-embedded 3-dimensional disc in $\mathcal{B}_{I X}$ which depends continuously on $q$. Moreover, the orbit of every point of $D_{\mathrm{IX}}^{s}(q)$ is the orbit of a point of $W_{\varepsilon}^{s}(\Theta, \bar{q}) \cap \mathcal{B}_{\mathrm{IX}}$. So, claim 1 and 2 are still satisfied if we replace $r \in W_{\varepsilon}^{s}(\Theta, \bar{q})$ by $r \in D^{s} \operatorname{IX}(q)$.

To complete the proof of our main theorem 1.3, it remains to show that the disc $D_{\mathrm{IX}}^{s}(q)$ depends continuously on $q$. This follows from the continuity of the maps $q \mapsto W_{\varepsilon}^{s}(\Theta, \bar{q})$ (theorem 6.6) and $q \mapsto \bar{q}$ (proposition 4.2).

\section{EXISTENCE OF CLOSED $f$-INVARIANT APERIODIC SUBSETS OF THE KASNER CIRCLE}

The purpose of this section is to prove proposition 1.5. This proposition should be quite obvious for people with some culture in dynamical systems. Indeed, the Kasner map $f: \mathcal{K} \rightarrow \mathcal{K}$ is a degree -2 map of the circle $\mathcal{K}$. This implies the existence of a continuous degree 1 map $\phi: \mathcal{K} \rightarrow \mathbb{R} / \mathbb{Z}$ such that $\phi \circ f=m \circ \phi$ where $m: \mathbb{R} / \mathbb{Z} \rightarrow \mathbb{R} / \mathbb{Z}$ is defined by $m(\theta)=-2 \theta$. Moreover, the Kasner map $f$ is expansive : the norm of the derivative of $f$ (calculated with respect to the metric induced on $\mathcal{K}$ by $h=d \Sigma_{1}^{2}+d \Sigma_{2}^{2}+d \Sigma_{3}^{2}+d N_{1}^{2}+d N_{2}^{2}+d N_{3}^{2}$ ) is strictly bigger than 1 , except at the three special points $T_{1}, T_{2}, T_{3}$ (where it is equal to 1 ). This implies that the map $\phi: \mathcal{K} \rightarrow \mathbb{R} / \mathbb{Z}$ defined above is one-to-one, that is $f: \mathcal{K} \rightarrow \mathcal{K}$ is topologically conjugated to the map $\theta \mapsto-2 \theta$. Finally, it is well-known by experts that, for $|k| \geq 2$, the union of all compact subsets of $\mathbb{R} / \mathbb{Z}$ which are aperiodic for the map $\theta \mapsto k \theta$ is dense in $\mathbb{R} / \mathbb{Z}$. We now give a more detailed proof of the proposition for the readers who may not necessarily be familiar with low-dimensional dynamics.

Proof. Recall that $T_{1}, T_{2}, T_{3}$ are the three Taub points on the Kasner circle $\mathcal{K}$. Let $I_{1}, I_{2}, I_{3}$ be the closures of the three connected components of $\mathcal{K} \backslash\left\{T_{1}, T_{2}, T_{3}\right\}$, the notations being chosen so that $T_{1}$ is not one end of $I_{1}, T_{2}$ is not one end of $I_{2}$, and $T_{3}$ is not an end of $I_{3}$. We denote by dist the distance on $\mathcal{K}$ induced by $h=d \Sigma_{1}^{2}+d \Sigma_{2}^{2}+d \Sigma_{3}^{2}+d N_{1}^{2}+d N_{2}^{2}+d N_{3}^{2}$; the norm of the derivative of $f$ will be calculated with respect to this distance.

We consider the set $\Sigma:=\{1,2,3\}^{\mathbb{N}}$ endowed with the product topology, and the shift map $\sigma: \Sigma \rightarrow \Sigma$ defined by $\sigma\left(a_{0}, a_{1}, a_{2}, \ldots\right)_{i}=\left(a_{1}, a_{2}, a_{3}, \ldots\right)$ (in other words, if $\bar{a}=\left(a_{i}\right)_{i \in \mathbb{N}} \in \Sigma$, then $(\sigma(\bar{a}))_{i}=$ $\left.a_{i+1}\right)$. Let $\Sigma_{0}$ be the subset of $\Sigma$ defined as follows :

$$
\Sigma_{0}=\left\{\bar{a}=\left(a_{i}\right)_{i \in \mathbb{N}} \in \Sigma \text { such that } a_{i+1} \neq a_{i} \text { for every } i\right\} .
$$

Note that $\Sigma_{0}$ is $\sigma$-invariant. We will construct a continuous "almost one-to-one" map $\phi: \Sigma_{0} \rightarrow \mathcal{K}$ such that $\phi \circ \sigma=f \circ \phi$.

Claim. For each sequence $\bar{a}:=\left(a_{i}\right)_{i \in \mathbb{N}}$ in $\Sigma_{0}$, there exists a unique point $x \in \mathcal{K}$ such that $f^{i}(x) \in I_{a_{i}}$ for every $i \geq 0$.

In order to prove the existence of $x$, one just needs to notice that the image under $f$ of each of the intervals $I_{1}, I_{2}, I_{3}$ is the union of the two other intervals. This implies that the intersection $\bigcap_{i=0}^{N} f^{-i}\left(I_{a_{i}}\right)$ is non-empty for every $N$, and therefore, that the intersection $\bigcap_{i \in \mathbb{N}} f^{-i}\left(I_{a_{i}}\right)$ is non-empty. The existence of $x$ follows. In order to prove the uniqueness of $x$, observe that: for every $\varepsilon>0$, there exists $\alpha(\varepsilon)>0$ such that $\left\||D f(x) \||_{h} \geq 1+\alpha(\varepsilon)\right.$ for every $x \in \mathcal{K}$ such that $\operatorname{dist}\left(x, T_{i}\right)>\varepsilon$ for $i=1,2,3$. Hence, if $x \neq x^{\prime}$ were two points such that $f^{i}(x) \in I_{a_{i}}$ and $f^{i}\left(x^{\prime}\right) \in I_{a_{i}}$ for every $i \geq 0$, then one would have 
$\operatorname{dist}\left(f^{i}(x), f^{i}\left(x^{\prime}\right)\right) \rightarrow \infty$; this is absurd since the lengths of $I_{1}, I_{2}$ and $I_{3}$ are finite. Hence there is at most one point $x$ in $\bigcap_{i \in \mathbb{N}} f^{-i}\left(I_{a_{i}}\right)$. This completes the proof of the claim.

Now, we consider the map $\phi: \Sigma_{0} \rightarrow \mathcal{K}$ which maps a sequence $\bar{a}:=\left(a_{i}\right)_{i \in \mathbb{N}}$ to the unique point $x \in \mathcal{K}$ such that $f^{i}(x) \in I_{a_{i}}$ for every $i \geq 0$. This map $\phi$ obviously satisfies $\phi \circ \sigma=f \circ \phi$. It is continuous (this is an immediate consequence of the continuity of $f$ ) and onto (because the image under $f$ of one of the intervals $I_{1}, I_{2}, I_{3}$ is contained in the union of the two others). It is not one-to-one. For example, the Taub point $T_{3}$ has two pre-images under $\phi$ : the sequences $(1,2,1,2,1,2, \ldots)$ and $(2,1,2,1,2,1, \ldots)$. More generally, every point $x \in \mathcal{K}$ such that $f^{i_{0}}(x)$ is a Taub point for some integer $i_{0} \geq 0$ (and thus $f^{i}(x)=f^{i_{0}}(x)$ for every $\left.i \geq i_{0}\right)$ has two pre-images under $\phi$, and these two pre-images are preperiodic for $\sigma$. This is the only lack of injectivity of $\phi:$ if $x \in \mathcal{K}$ has not a single pre-image under $\phi$, then there exists $i_{0} \geq 0$ such that $f^{i_{0}}(x)$ is a Taub point (this follows from the fact that the intersection between two of the three intervals $I_{1}, I_{2}, I_{3}$ is reduced to a Taub point).

It follows that the image under $\phi$ of a closed $\sigma$-invariant aperiodic subset of $\Sigma_{A}$ is a closed $f$-invariant aperiodic subset of $\mathcal{K}$. So we are left to prove that the union of all the closed $\sigma$-invariant and aperiodic subsets of $\Sigma_{A}$ is in dense in $\Sigma_{A}$.

A element $\bar{a}=\left(a_{i}\right)_{i \geq 0}$ of $\Sigma_{0}$ is said to be square-free if it does not contain the same word repeated twice : for every $i_{0} \geq 0$ and every $\ell>0$, the word $a_{i_{0}} \ldots a_{i_{0}+\ell-1}$ is different from the word $a_{i_{0}+\ell} \ldots a_{i_{0}+2 \ell-1}$. It is well-known that there exist square-free elements in $\Sigma_{0}$ (such an element may be easily deduced from the well-known Prouhet-Thue-Morse sequence, see for example [1, corollary 1]). Now let $\bar{a}=\left(a_{i}\right)_{i \geq 0} \in \Sigma_{0}$ be square-free, then the $\sigma$-orbit of $\bar{a}$ does not accumulates on any periodic $\sigma$-orbit, hence the closure of the $\sigma$-orbit of $\bar{a}$ is a closed, positively invariant aperiodic subset of $\Sigma_{0}$. Moreover the same is true, if one replaces $\bar{a}$ by a sequence $\bar{a}^{\prime} \in \Sigma_{0}$ which has the same tail as $\bar{a}$ (i.e. there exists $i_{0}$ such that $a_{i}^{\prime}=a_{i}$ for $i \geq i_{0}$ ). The set of all sequences $\bar{a}^{\prime}$ which has the same tail has $\bar{a}$ is obviously dense in $\Sigma_{0}$. Hence the union of all closed $\sigma$-invariant aperiodic subsets of $\Sigma_{0}$ is dense in $\Sigma_{0}$. As explained above, the proposition follows.

\section{REFERENCES}

[1] J.-P. Allouche and J. Shallit. The ubiquitous Prouhet-Thue-Morse sequence. Sequences and their applications, Proceedings of SETA98, C. Ding, T. Helleseth and H. Niederreiter Eds (1999), Springer Verlag, 116.

[2] M. Georgi, J. Härterich, S. Liebscher and K. Webster. Ancient Dynamics in Bianchi Models. Approach to Periodic Cycles. arXiv: 1004.1989.

[3] J. M. Heinzle and C. Uggla. Mixmaster : Fact and Belief.. arXiv: 0901.0776.

[4] J. M. Heinzle and C. Uggla. A new proof of the Bianchi type IX attractor theorem. arXiv:0901.0806.

[5] C. W. Misner. Mixmaster universe. Phys. Rev. Lett. 22 (1969), 1071-1074.

[6] J. Palis and F. Takens. Hyperbolicity and sensitive chaotic dynamics at homoclinic bifurcations. Cambridge University Press, 1993.

[7] A. Rendall. Global dynamics of the mixmaster model. Class. Quantum Grav. 14 (1997), 2341-2356.

[8] H. Ringstrom. Curvature blow up in Bianchi VIII and IX vacuum spacetimes. Class. Quantum. Grav. 17 (2000), $713-731$.

[9] H. Ringstrom. Bianchi IX attractor. Annales Henri Poincaré 2 (2001), 405-500.

[10] F. Takens. Partially hyperbolic fixed points. Topology 10 (1971), 133-147.

[11] J. Wainwright and G. F. R. Ellis. Dynamical Systems in Cosmology, Cambridge University Press, 1997.

Laboratoire de Mathématiques (UMR 8628), Univ. Paris Sud 11, 91405 Orsay, France.

E-mail address: Francois. Beguin@math.u-psud.fr 